\title{
Atmospheric lonizing Radiation from Galactic and Solar Cosmic Rays
}

\author{
Christopher J. Mertens ${ }^{1}$, Brian T. Kress ${ }^{2}$, Michael Wiltberger ${ }^{3}$, W. Kent \\ Tobiska ${ }^{4}$, Barbara Grajewski ${ }^{5}$ and Xiaojing $\mathrm{Xu}^{6}$ \\ ${ }^{1}$ NASA Langley Research Center, Hampton, Virginia \\ ${ }^{2}$ Dartmouth College, Hanover, New Hampshire \\ ${ }^{3}$ High Altitude Observatory, National Center for Atmospheric Research, Boulder, Colorado \\ 4 Space Environment Technologies, Pacific Palisades, California \\ ${ }^{5}$ National Institute for Occupational Safety and Health, Cincinnati, Ohio \\ ${ }^{6}$ Science Systems and Applications, Inc. \\ USA
}

\section{Introduction}

An important atmospheric state variable, driven by space weather phenomena, is the ionizing radiation field. The two sources of atmospheric ionizing radiation are: (1) the ever-present, background galactic cosmic rays (GCR), with origins outside the solar system, and (2) the transient solar energetic particle (SEP) events (or solar cosmic rays), which are associated with eruptions on the Sun's surface lasting for several hours to days with widely varying intensity. Quantifying the levels of atmospheric ionizing radiation is of particular interest to the aviation industry since it is the primary source of human exposure to high-linear energy transfer (LET) radiation. High-LET radiation is effective at directly breaking DNA strands in biological tissue, or producing chemically active radicals in tissue that alter the cell function, both of which can lead to cancer or other adverse health effects (Wilson et al., 2003; 2005b). Studies of flight attendants have suggested adverse reproductive health outcomes (Aspholm et al., 1999; Lauria et al., 2006; Waters et al., 2000). The International Commission on Radiological Protection (ICRP) classify crews of commercial aircraft as radiation workers (ICRP, 1991). The US National Council on Radiation Protection and Measurements (NCRP) reported that among radiation workers monitored with recordable dose, the largest average effective dose in 2006 ( $3.07 \mathrm{mSv}$ ) was found in flight crew. In contrast, the average for the workers with the second largest effective dose, commercial nuclear power workers, was $1.87 \mathrm{mSv}$ (NCRP, 2009). However, aircrew are the only occupational group exposed to unquantified and undocumented levels of radiation. Furthermore, the current guidelines for maximum public and prenatal exposure can be exceeded during a single solar storm event for commercial passengers on intercontinental or cross-polar routes, or by frequent use $(\sim 10-20$ flights per year) of these high-latitude routes even during background conditions (AMS, 2007; Copeland et al., 2008; Dyer et al., 2009).

There is an important national need to understand and to predict the real-time radiation levels for the commercial aviation industry and the flying public, which has broad societal, public health, and economic benefits. NASA has met this need by developing the 
first-ever, real-time, global, physics-based, data-driven model for the prediction of biologically hazardous atmospheric radiation exposure. The model is called Nowcast of Atmospheric Ionizing Radiation for Aviation Safety (NAIRAS). In this chapter the underlying physics of the NAIRAS model is reviewed and some of the key results and applications of the model are presented. More specifically, the materials reviewed are the latest understanding in the physics and transport of atmospheric ionizing radiation from galactic and solar cosmic rays, the influence of space weather on the atmospheric ionizing radiation field, basic radiation dosimetry applied to atmospheric exposure, and the latest epidemiological understanding of radiation effects on pilots and aircrew.

\section{Scientific and historical overview}

\subsection{Basics on cosmic rays and matter interactions}

GCR consist of roughly $90 \%$ protons and $8 \%$ helium nuclei with the remainder being heavier nuclei and electrons (Gaisser, 1990). When these particles penetrate the magnetic fields of the solar system and the Earth and reach the Earth's atmosphere, they collide with air molecules and create cascades of secondary radiations of every kind (Reitz et al., 1993). The collisions are primarily due to Coulomb interactions of the GCR particle with orbital electrons of the air molecules, delivering small amounts of energy to the orbital electrons and leaving behind electron-ion pairs (Wilson et al., 1991). The ejected electrons usually have sufficient energy to undergo similar ionizing events. The cosmic ray ions lose a small fraction of their energy and must suffer many of these atomic collisions before slowing down. On rare occasions the cosmic ray ion will collide with the nucleus of an air molecule in which large energies are exchanged and the ion and nucleus are dramatically changed by the violence of the event. The remnant nucleus is highly disfigured and unstable, emitting further air nuclear constituents and decaying through the usual radioactivity channels (Wilson et al., 1991). One of the most important secondary particles created in GCR-air interactions is the neutron. Because of its charge neutrality, the neutron penetrates deep into the atmosphere, causing further ionization events along its path and contributing over half the atmospheric radiation exposure at typical commercial airline altitudes (Wilson et al., 2003). Furthermore, neutron exposures pose a relatively high health risk, since the massive low-energy ions resulting from neutron interactions always produce copious ions in the struck cell and repair is less efficient for these events (Wilson, 2000).

The intensity of the atmospheric radiations, composed of GCR primary and secondary particles, their energy distribution, and their effects on aircraft occupants vary with altitude, location in the geomagnetic field, and the time in the sun's magnetic activity (solar) cycle (Heinrich et al., 1999; Reitz et al., 1993; Wilson, 2000). The atmosphere provides material shielding, which depends on the overhead atmospheric depth. The geomagnetic field provides a different kind of shielding, by deflecting low-momentum charged particles back to space. Because of the orientation of the geomagnetic field, which is predominately dipolar in nature, the polar regions and high latitudes are susceptible to penetrating GCR (and SEP) particles. At each geographic location, the minimum momentum per unit charge (magnetic rigidity) a vertically incident particle can have and still reach a given location above the earth is called the vertical geomagnetic cutoff rigidity. The local flux of incident GCR at a given time varies widely with geomagnetic location and the solar modulation level. When solar activity is high, GCR flux is low, and vice versa. The dynamical balance between outward convective flux of solar wind and the inward diffusive flux of GCR is responsible for the anti-correlation 
between the incident GCR and the modulation level of solar cycle activity (Clem et al., 1996; Parker, 1965).

It is now generally understood that SEP events arise from coronal mass ejections (CME) from active regions on the solar surface (Kahler, 2001; Wilson et al., 2005b). The CME propagates through interplanetary space carrying along with it the local surface magnetic field frozen into the ejected mass. There is a transition (shock) region between the normal sectored magnetic structure of interplanetary space and the fields frozen into the ejected mass, where the interplanetary gas is accelerated forming the SEP. As the accelerated region passes an observation point, the flux intensity is observed to increase dramatically, and no upper limit in intensity is known within the shock region. The SEP energy spectrum obtained in the acceleration process is related to the plasma density and CME velocity. During a solar storm CME event, the number flux distribution incident at Earth's atmosphere is a combination of the GCR and SEP distributions. The SEP-air interaction mechanisms are the same as GCR-air interactions described above. The atmospheric radiations caused by a SEP also vary with altitude and geomagnetic field.

\subsection{Commercial aircraft radiation exposure}

GCR radiations that penetrate the atmosphere and reach the ground are low in intensity. However, the intensities are more than two orders of magnitude greater at commercial aircraft altitudes. At the higher altitudes of High Speed Civil Transport (HSCT), the GCR intensity is another two orders of magnitude higher (Wilson et al., 2003). When the possibility of high-altitude supersonic commercial aviation was first seriously proposed (The Supersonic Transport program proposed in 1961), Foelsche brought to light a number of concerns about associated atmospheric radiation exposure due to GCR and SEP, including the secondary radiations (Foelsche, 1961; Foelsche \& Graul, 1962). Subsequently, Foelsche et al. (1974) conducted a detailed study of atmospheric ionizing radiation at high altitudes from 1965 to 1971 at the NASA Langley Research Center (LaRC). The study included a comprehensive flight program in addition to theoretical investigations. The measured data and theoretical calculations were integrated into a parametric Atmospheric Ionizing Radiation (AIR) model (Wilson et al., 1991). Prior to that study the role of atmospheric neutrons in radiation exposure was generally regarded as negligible (Upton et al., 1966). The LaRC studies revealed neutron radiation to be a major contributor to aircraft GCR exposure. Still the exposure levels were comfortably below allowable exposure limits for the block hours typical of airline crews of that time, except during a possible SEP event (less than 500 block hours were typical of the 1960 's, although regulation allowed up to 1000 hours). Assessments of radiation exposure extending back to the 1930's for former Pan Am flight attendants were conducted by Waters et al. (2009) and Anderson et al. (2011).

There have been a number of significant changes since the original work of Foelsche (Wilson et al., 2003). A partial list of these changes, relevant to the development of the NAIRAS model, are: (1) the highly ionizing components of atmospheric radiations are found to be more biologically damaging than previously assumed and the associated relative biological effectiveness for fatal cancer has been increased (ICRP, 1991; ICRU, 1986); (2) recent animal and human studies indicate large relative biological effectiveness requiring protection for reproductive exposure (BEIR V, 1990; Chen et al., 2005; Fanton \& Gold, 1991; Jiang et al., 1994; Ogilvy-Stuart \& Shalet, 1993); (3) recent epidemiological studies (especially the data on solid tumors) and more recent atom-bomb survivor dosimetry have resulted in higher radiation risk coefficients for gamma rays (ICRP, 1991; NAS/NRC, 1980; UNSCEAR, 1988), resulting in 
lower proposed permissible limits (ICRP, 1991; NCRP, 1993); (4) subsequent to deregulation of the airline industry, flight crews are logging greatly increased hours (Barish, 1990; Bramlitt, 1985; Friedberg et al., 1989; Grajewski et al., 2011; Wilson \& Townsend, 1988); and (5) airline crew members are now classified by some agencies as radiation workers (ICRP, 1991).

The last point (i.e., (5)) is particularly illuminating. Aircrews may even receive exposures above recently recommended allowable limits for radiation workers when flying the maximum allowable number of flight hours. Reviews (Hammer et al., 2009) and meta-analyses (Buja et al., 2005; 2006) of cancer studies among flight crew have found excesses of breast cancer and melanoma and possible excesses for other sites. Increasing risk trends with radiation exposure have not been consistently identified, likely due to the limitations of the exposure metrics used (Waters et al., 2000). There is further concern for reproductive health from exposure to high altitude and/or high-latitude flights, as the US National Institute for Occupational Safety and Health (NIOSH) continues to study adverse pregnancy outcomes among commercial flight attendants (Waters et al., 2000). US pilots have been exposed to increasing levels of ionizing radiation since the 1990s (Grajewski et al., 2011). Frequent-flyer business passengers are likely exposed to even higher doses than aircrew, since flight hours are not restricted for airline passengers. In addition, if a large SEP occurs during flight, both passengers and pregnant crew may greatly exceed allowable limits (Barish, 2004).

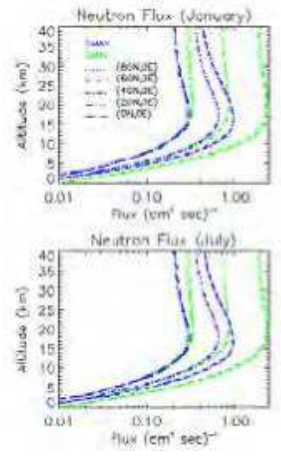

(a) AIR Neutron Flux and Ionization Rates
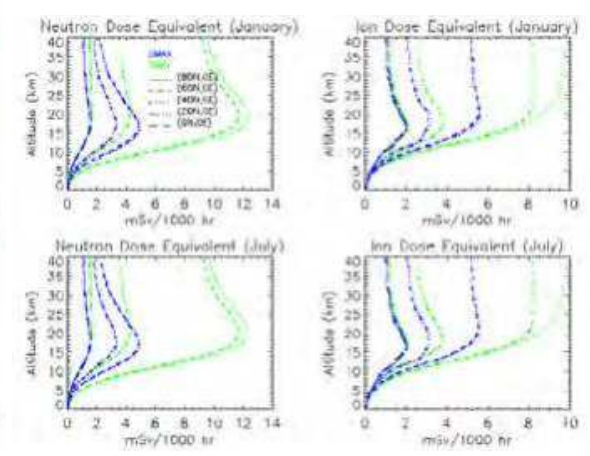

(b) AIR Dose Equivalent Rates

Fig. 1. (a) Neutron flux and ionization rate profiles for various latitudes, solar activity levels, and seasons. The blue lines represent solar maximum conditions. The green lines represent solar minimum conditions. The top row corresponds to January atmospheric conditions while the bottom row corresponds to July. (b) Neutron and ion dose equivalent rates profiles for the same latitudes, solar activity levels, and seasons indicated in (a).

The original LaRC study (1965 to 1971) commissioned over 300 flights over most of the duration of solar cycle 20 on high-altitude aircraft and balloons to study both the background radiation levels over the solar cycle and to make measurements during SEP events. The LaRC flight package consisted of a 1-10 MeV neutron spectrometer, tissue equivalent ion chamber, and nuclear emulsion for nuclear reaction rates in tissue. Monte Carlo calculations (Lambiotte et al., 1971; Wilson et al., 1970) for incident GCR protons were used to extend the neutron spectrum to high energies. The measured data were combined with the theoretical calculations and integrated into the parametric AIR model, parameterized by neutron monitor count rate, vertical geomagnetic cutoff rigidity, and atmospheric depth. Solar cycle modulation of the GCR spectrum is parameterized by the ground-level neutron 
monitor count rates. Geomagnetic momentum shielding and overhead atmospheric shielding are parameterized by the vertical geomagnetic cutoff rigidity and atmospheric depth, respectively. The neutron flux $\left(\mathrm{cm}^{-2} \mathrm{sec}^{-1}\right)$ component to the atmospheric radiations is converted to dose equivalent rate and total dose rate using $3.14 \mathrm{~Sv} \mathrm{~cm}^{2} \mathrm{sec} \mathrm{hr}^{-1}$ and $0.5 \mathrm{~Gy}$ $\mathrm{cm}^{2} \mathrm{sec} \mathrm{hr}^{-1}$, respectively. The charged particle component of the atmospheric radiations is obtained from data taken by Neher $(1961 ; 1967 ; 1971)$ and Neher \& Anderson $(1962)$ as compiled S. B. Curtis (Boeing 1969) and utilized by Wallance \& Sondhaus (1978). The charge particle atmospheric ionization rates are directly converted to dose equivalent rate and total dose rate using measurement data from the tissue equivalent ion chamber. Nuclear stars in tissue are estimated from the nuclear emulsion measurement data after subtraction of the neutron-induced stars (Wilson et al., 1991). Recent updates to the parametric AIR model are described by Mertens et al. (2007a).

Figure 1a shows altitude profiles of neutron flux and ionization rates computed from the AIR model for summer and winter seasons at various latitudes in the northern hemisphere, for both solar maximum and solar minimum conditions. Figure $1 \mathrm{~b}$ shows the corresponding profiles of dose equivalent rates. In this context, dose rate refers to the rate at which radiation energy is absorbed in human tissue per unit mass. The unit of dose is Gray $\left(1 \mathrm{~Gy}=6.24 \times 10^{12}\right.$ $\mathrm{MeV} \mathrm{kg}{ }^{-1}$ ). Dose equivalent rate is the sum of the dose (Gy) from each radiation particle, which each particle dose weighted by a factor related to the potential to inflict biological damage (Mertens et al., 2007a). The unit of dose equivalent is Sievert (Sv). Dose equivalent is closely related to biological risk due to radiation exposure (Wilson, 2000). The dosimetric quantities are discussed in more detail in section 3.6.

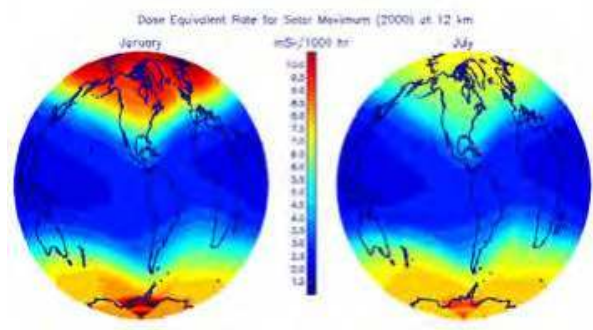

(a) AIR Dose Equivalent Rates: Solar Max

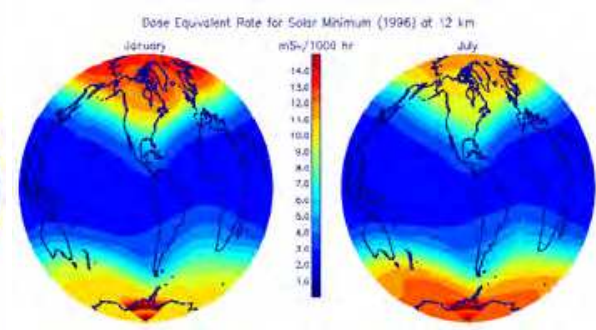

(b) AIR Dose Equivalent Rates: Solar Min

Fig. 2. (a) Global distribution of dose equivalent rate (mSv/1000 hr) predicted by the parametric AIR model at $12 \mathrm{~km}$ for solar maximum conditions (year 2000) of cycle 23. (b) The same as in (a) but for solar minimum conditions (year 1996) of cycle 22.

The two most noticeable features in Figure 1 are: (1) the significant increase in flux, ionization rates, and dose equivalent rates at high-latitudes, and (2) the peak in these quantities occur near the typical cruising altitudes of commercial aircraft flying international routes $(\sim 10-12$ $\mathrm{km}$ ). The low altitude results are less reliable because of the limited altitude range of the balloon and flight measurements used to develop the AIR parameterizations. The first point is underscored in Figure 2, which shows global maps of the dose equivalent rates computed from the parametric AIR model for summer and winter northern hemisphere seasons at 12 $\mathrm{km}$ for both solar minimum and solar maximum conditions. The solar cycle variation in the dose equivalent rates is governed by modulation of the local interstellar spectrum of GCR particles by the heliospheric solar wind and interplanetary magnetic field (Mertens et al., 
2008; 2007a; Wilson et al., 2003; 1991). The latitudinal variation in the dose equivalent rates is determined by the low-momentum shielding provided by the Earth's magnetic field (Mertens et al., 2010a; 2009; 2008; 2007a; Wilson et al., 2003; 1991). The seasonal variations in the dose equivalent rates are determined by the seasonal variations in the overhead atmospheric mass at a fixed altitude (Mertens et al., 2008; 2007a; Wilson et al., 2003; 1991). These space weather and meteorological influences on atmospheric radiation exposure are discussed more fully in sections 3 and 4 .

An important point to note is that the North Atlantic corridor region is one of the busiest in the world and comprises the most highly exposed routes in airline operations. This is clearly seen in Figure 2. High-latitude flights, polar flights, and flights over Canada are among the most highly exposed. The maximum GCR radiation exposure occurs during solar minimum conditions. The annual level of GCR exposure to pilots flying high-latitude routes and logging maximum flight hours (1000-hours) is sufficient to trigger individual monitoring and medical surveillance in the European Union (EU) states. Low latitude flights, on the other hand, are minimally exposed to cosmic ray radiation.

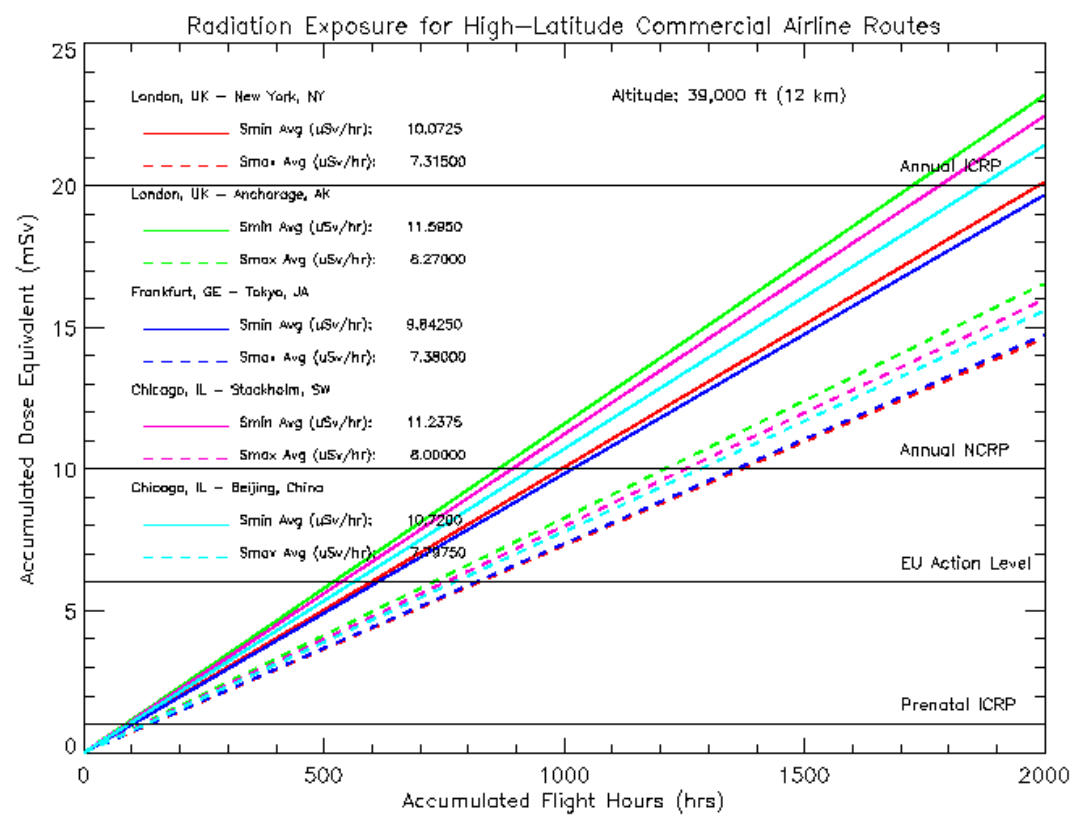

Fig. 3. Accumulated dose equivalent $(\mathrm{mSv})$ predicted by the parametric AIR model as a function of accumulated flight time (hrs) at $12 \mathrm{~km}$ for representative high-latitude commercial routes. The solid lines denote solar minimum conditions (year 1996) while the dashed lines denote solar maximum (year 2000) conditions. The top two horizontal lines indicate the recommended ICRP and NCRP annual exposure limits for radiation workers, respectively. The bottom horizontal line shows the ICRP recommended prenatal and annual public exposure limits. The "EU Action Level" horizontal line is described in section 2.3 


\subsection{The NAIRAS decision support system}

To place biological risk to high-latitude commercial aircrew and passengers from high-LET radiation exposure into context, consider the accumulated dose equivalent shown in Figure 3 for representative high-latitude commercial routes. The accumulated dose equivalent in Figure 3 is from GCR radiation exposure predicted by the parametric AIR model for both solar maximum and solar minimum conditions. The ICRP recommends $20 \mathrm{mSv}$ as the annual occupational radiation worker limit (ICRP, 2008; 1991; Wilson, 2000; Wilson et al., 2003). The National Committee on Radiological Protection (NCRP) recommends $10 \mathrm{mSv}$ as the annual occupational radiation worker limit (a conservative reconciliation of the NCRP $10^{*}$ age occupational lifetime limit with its annual occupational limit of 50mSv/yr; see Wilson (2000); Wilson et al. (2003).). The recommended ICRP annual public limit and prenatal exposure limit is $1 \mathrm{mSv}$. These limits are shown as horizontal black lines in Figure 3.

The Council of the EU adopted Directive 96/20/EURATOM on 13 May 1996. Article 42 of the EU Directive imposes requirements relating to the assessment and limitation of aircrew cosmic ray radiation exposure (AMS, 2007). EU Member States were required to implement the Directive by 13 May 2000 through national legislation. Consistent with "best practice" radiation protection procedures, which is to keep all radiation exposures as low as reasonably achievable (i.e., the ALARA principle), the EU has also adopted an "action level" of $6 \mathrm{mSv} / \mathrm{yr}$. The EU action level is also shown as a horizontal black line in Figure 3. For those likely to exceed $6 \mathrm{mSv} / \mathrm{yr}$, individual record keeping and medical surveillance is required of the aircraft operators (Dyer \& Lei, 2001). For exposures less than $6 \mathrm{mSv} / \mathrm{yr}$, monitoring is only recommended and the actual implementation of these recommendation varies among the EU Member States (Meier et al., 2009). The EU Directive recommendation is that individual exposure is assessed by the aircraft operators if one is likely to exceed $1 \mathrm{mSv} / \mathrm{yr}$, and that workers are educated on radiation health risks and work schedules are adjusted to ensure that the $6 \mathrm{mSv} / \mathrm{yr}$ level is not exceeded (EURADOS, 1996).

The results from the parametric AIR model in Figure 3 indicate that high-latitude flying commercial aircrew will trigger the "EU action level" for annual flight hours more than $\sim 500-600$ hours during solar minimum and more than $\sim 800-900$ hours during solar maximum conditions. The recommended ICRP annual public and prenatal limit is quite low. From Figure 3, the public/prenatal limit can be exceeded in 100 hours of flight time. For high-latitude and polar flights, the dose equivalent rate is on the order of $\sim 10 \mathrm{uSv} / \mathrm{hr}$ (Copeland et al., 2008; Dyer et al., 2009; Mertens et al., 2008; 2007a). An accumulated 100 hours of flight time can be accrued from 10-20 international flights with roughly 5-10 hours of flight time per flight. Thus, at an average high-latitude GCR exposure rate of $10 \mathrm{uSv} / \mathrm{hr}$, the ICRP annual public and prenatal limit can be exceeded in 10-20 flights.

Recognizing the potential impact on present day passenger and crew exposures - due to the changes since the original work of Foelsche, as described in section 2.2, combined with the results shown in Figure 3 - further studies were initiated at LaRC. A new radiation measurement flight campaign was formulated and executed, consisting of a collaboration of fourteen institutions in five countries with a contribution of eighteen instruments to the flight program. New measurements and advances in theoretical modeling followed (Clem et al., 1996; Wilson et al., 2003), which culminated in the AIR workshop (Wilson et al., 2003).

Following the recent LaRC-sponsored AIR workshop, a number of recommendations for future work were put forth (Wilson et al., 2003). The recommendations relevant to the NAIRAS model development are: (1) utilize satellite input data to provide real-time mapping 
of GCR and SEP radiation levels to provide guidance in exposure avoidance; and (2) utilize state-of-the-art transport codes and nuclear databases to generate input data to the AIR model. The NAIRAS model addresses these two recommendations, but significantly goes beyond recommendation (2) by using physics-based, state-of-the-art transport code directly in simulating the atmospheric radiation exposure levels. The details of the NAIRAS model are given in section 3 .

There are also economic consequences to the issue of aircraft radiation exposure. Over the last decade, airspace over Russian and China has opened up to commercial traffic, allowing for polar routes between North America and Asia (AMS, 2007). These cross-polar routes reduce flight time and operational cost; thus, the number of cross-polar commercial routes has increased exponentially. The typical cost savings from a cross-polar route from the US to China is between $\$ 35,000$ and $\$ 45,000$ per flight compared to the previous non-polar route (DOC, 2004). However, the polar region receives the largest quantity of radiation because the shielding provided by Earth's magnetic field rapidly approaches zero near the magnetic pole. On the other hand, the economic loss to an airline from rerouting a polar flight in response to an SEP warning, for example, can be a factor of three greater than the original cost-savings of flying the polar route if fuel stops and layovers are necessary. Thus, the cost to reroute a cross-polar route can be as much as $\$ 100,000$ per flight (DOC, 2004). Consequently, an aircraft radiation prediction model must also be accurate to minimize radiation risks while simultaneously minimizing significant monetary loss to the commercial aviation industry.

The goal of the NAIRAS model is to provide a new decision support system for the National Oceanic and Atmospheric Administration (NOAA) Space Weather Prediction Center (SWPC) that currently does not exist, but is essential for providing the commercial aviation industry with data products that will enable airlines to achieve the right balance between minimizing flight cost while at the same time minimizing radiation risk. NOAA/SWPC is the main provider of real-time space weather nowcasts and forecasts, both nationally and internationally. However, there are no existing data or models, within NOAA/SWPC or outside NOAA/SWPC, that provide a comprehensive (i.e., comprehensive in terms of input observation data included and comprehensive in terms of the transport physics included in the real-time calculations), global, real-time assessment of the radiation fields that affect human health and safety. NAIRAS is the first-ever, global, real-time, data-driven model that predicts aircraft ionizing radiation exposure, including both GCR and SEP exposures. Thus, the NAIRAS model addresses an important national and international need with broad societal, public health and economic benefits. A detailed description of the NAIRAS model is given in the next section.

\section{Description of NAIRAS model components}

NAIRAS is an operational model for predicting aircraft radiation exposure from galactic and solar cosmic rays (Mertens et al., 2010a; 2009; 2008; 2007a). The NAIRAS prototype model development was funded by the NASA Applied Sciences / Aviation Weather Program. Real-time exposure rate graphical and tabular data products from the operational prototype are streaming live from the NAIRAS public web site at http://sol.spacenvironment.net/ nairas/ (or, just google NAIRAS to locate web site). A subset of the NAIRAS real-time graphical products are available on the SpaceWx smartphone app for iPhone, IPad, and Android.

NAIRAS provides data-driven, global, real-time predictions of atmospheric ionizing radiation exposure rates on a geographic $1 \times 1$ degree latitude and longitude grid from the surface of 
the Earth to $100 \mathrm{~km}$ with a vertical resolution of $1 \mathrm{~km}$. The real-time, global predictions are updated every hour. NAIRAS has adopted, as far as possible, the meteorological weather forecasting paradigm of combining physics-based forecast models with data assimilation techniques. Physics-based models are utilized within NAIRAS to transport cosmic rays through three distinct material media: the heliosphere, Earth's magnetosphere, and the neutral atmosphere. While the quantity of observations relevant to radiation exposure predictions is currently too sparse to apply data assimilation techniques per se, nevertheless, as much real-time measurement data as possible are utilized. The real-time measurement data are used to: (1) specify the ionizing radiation field at the boundaries of the aforementioned material media, and (2) characterize the internal properties of the aforementioned material media. The real-time measurements provide necessary observational constraints on the physics-based models that improve simulations of the transport and transmutations of cosmic ray radiation through the heliosphere, magnetosphere, and atmosphere.

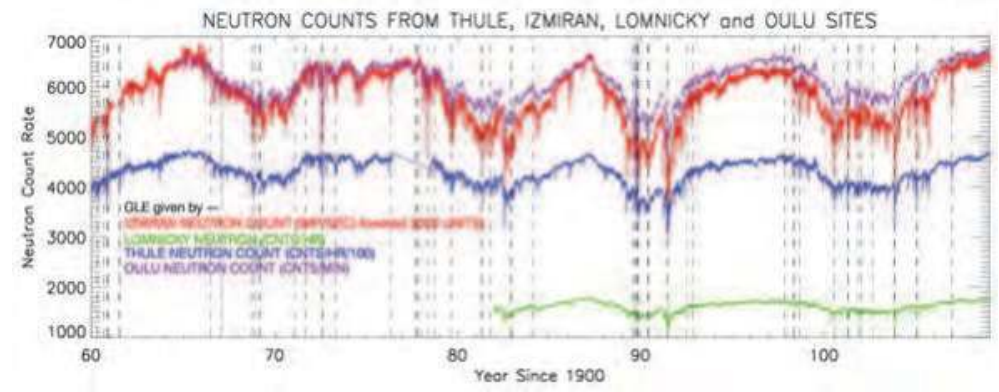

(a) Neutron Monitor Count Rates

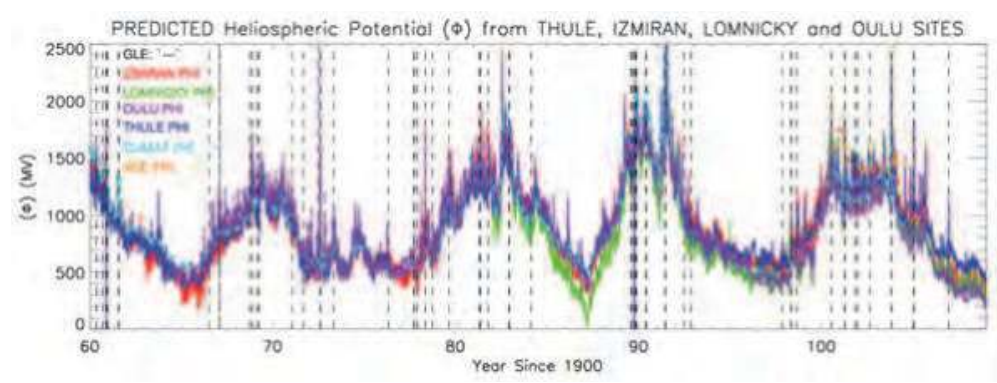

(b) Solar Modulation Parameter

Fig. 4. (a) Monthly-mean neutron monitor count rates from Thule, Izmiran (or Moscow), Lomnicky, and Oulu sites. The vertical dashed lines show ground level enhancement (GLE) events. The time period is 1960-2005. (b) Monthly-mean solar modulation parameter $(\Phi(t))$ predicted by the monthly-mean neutron monitor count rates shown in (a). The reference solar modulation parameters $\Phi_{\text {CLIMAX }}$ and $\Phi_{\text {ACE }}$ are also shown.

There are a number of models currently in use for calculating GCR radiation exposure at aircraft altitudes. The CARI-6 model utilizes a database of transport calculations generated by the deterministic LUIN code for a wide variety of geographic locations, altitudes, and solar activity levels (O'Brien et al., 1998; 2003). The EPCARD model is based on a similar approach, but uses the Monte Carlo FLUKA code for the transport calculations (Schraube 
et al., 1999). PC-AIRE is a semi-empirical model based on fits to measurement data (Lewis et al., 2002). Other aircraft radiation exposure models are described in the recent European Radiation Dosimetry Group report (Lindborg et al., 2004). Currently, the above models calculate SEP atmospheric radiation exposure post-storm on a case-by-case basis, although PC-AIRE incorporated low-earth orbit measurements to develop a simple extrapolation to SEP events (Lewis et al., 2002). Recently, Copeland et al. (2008) calculated adult and conceptus aircraft exposure rates for 170 SEP events for years 1986-2008 using the Monte Carlo MCNPX transport code.

The main differences that distinguish the NAIRAS model from the models discussed above are the following. Dynamical solar wind-magnetospheric interactions and the accompanying geomagnetic effects that govern the transport of cosmic rays through the magnetosphere are included in real-time in the NAIRAS radiation exposure calculations (Kress et al., 2004; 2010; Mertens et al., 2010a). Furthermore, the physics-based deterministic High Charge (Z) and Energy TRaNsport code (HZETRN) is used in transporting cosmic rays through the atmosphere. The HZETRN transport calculations are continuously updated using real-time measurements of boundary condition specifications of the space radiation environment and of atmospheric density versus altitude (Mertens et al., 2008; 2007a). And finally, both GCR and SEP atmospheric radiation exposure predictions are included in real-time (Mertens et al., 2010a; 2009).

The remainder of this section contains a detailed description of the salient features of the NAIRAS physics-based modules that transport cosmic rays through the heliosphere, Earth's magnetosphere, and the neutral atmosphere. The input measurement data to the physics-based modules, used to specify boundary conditions of the material medium or to characterize the internal properties of material medium through which the cosmic rays are transported, are also described. The first material medium considered is the heliosphere. GCR are transported from outside the heliosphere to $1 \mathrm{AU}$ using real-time measurements of ground-based neutron monitor count rates. This NAIRAS module is described in section 3.1. SEP are not transported per se but determined in the geospace environment in-situ using a combination of NOAA Geostationary Operational Environmental Satellite (GOES) and NASA Advanced Composition Explorer (ACE) ion flux measurements. This module is described in section 3.2. Both sources of cosmic rays, GCR and SEP, are transported through Earth's magnetosphere using a semi-physics-based geomagnetic shielding model. The geomagnetic shielding model utilizes real-time NASA/ACE solar wind and interplanetary magnetic field (IMF) measurements. This module is described in section 3.3. The cosmic rays that have passed through the heliosphere and magnetosphere are subsequently transported through the neutral atmosphere using the NASA LaRC HZETRN deterministic transport code. The internal properties of the atmosphere important to cosmic ray transport are provided in real-time by the global atmospheric mass density distribution obtained from the NOAA Global Forecasting System (GFS). These two NAIRAS modules are described in sections 3.4 and 3.5, respectively. Finally, the method of quantifying human exposure and the associated biological risk from the atmospheric ionizing radiation field is summarized in section 3.6.

\subsection{Heliospheric GCR transport}

GCR are transported through the heliosphere to the geospace environment using an expanded version of the Badhwar and O'Neill model (Badhwar \& O'Neill, 1991; 1992; 1993; 1994; Badhwar \& ONeill, 1996). The Badhwar and $\mathrm{O}^{\prime} \mathrm{Neill}$ model, which is simply referenced to as the GCR model, has been updated recently by $\mathrm{O}^{\prime} \mathrm{Neill}$ (2006) to use ground-based 
neutron monitor count rate measurements from the Climate neutron monitor site, in order to provide a measurement constraint on the simulated solar cycle modulation of the GCR spectrum at 1 AU. Comparisons between the GCR model and NASA/ACE measurements of the GCR spectra have shown that this step has enabled accurate predictions of GCR spectra in the geospace environment, at least on monthly to seasonal time scales (O'Neill, 2006). The NAIRAS team has extended the work of $\mathrm{O}^{\prime}$ Neill (2006) by incorporating four high-latitude neutron monitor count rate measurements into the GCR model predictions at 1 AU. These additional high-latitude neutron monitor stations are Thule, Oulu, Izmiran (or Moscow), and Lomnicky. The reasons for utilizing these neutron monitor data are two-fold: (1) high-latitude locations are sensitive to the GCR spectral region most influenced by solar cycle variability, and (2) the data from these stations are available in real-time or near real-time.

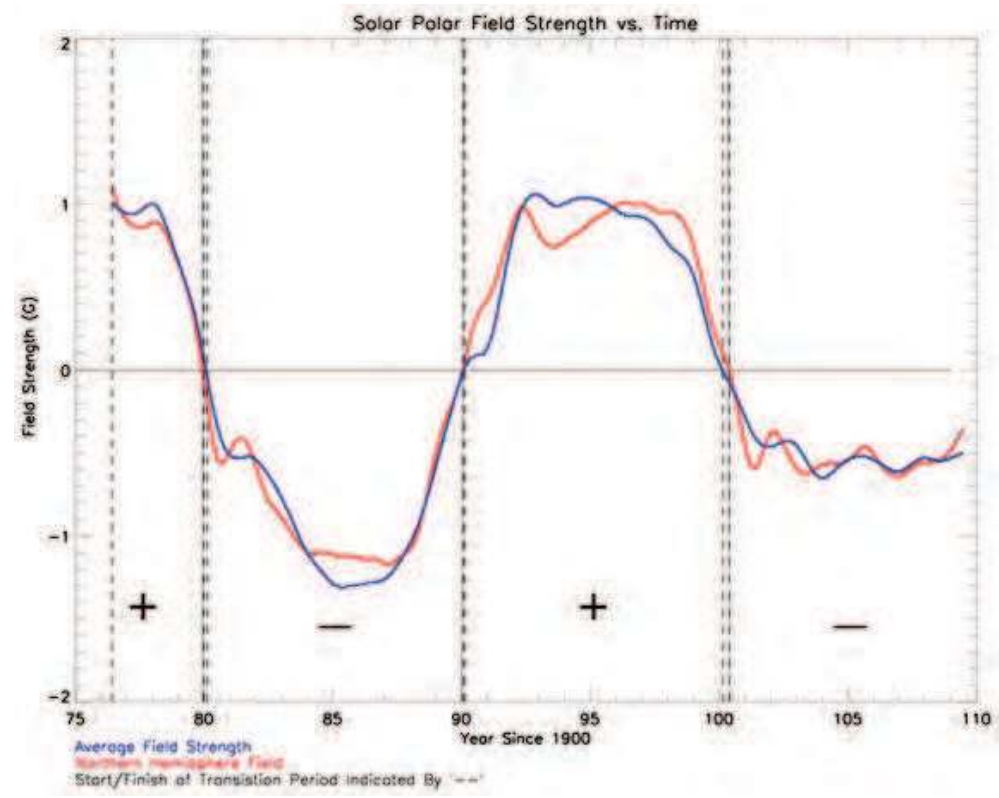

Fig. 5. Solar polar magnetic field data taken by measurements made at the Wilcox Solar Observatory. The blue line is the mean field strength of the northern and southern solar hemispheres. The red line is the northern hemisphere polar magnetic field strength. The ' + ' and '-' symbols between the vertical dashed lines indicate the time periods of solar positive and negative polarity, respectively. The transition region is the gap between the vertical dashed lines.

The GCR model propagates the local interstellar spectrum (LIS) of each element of the GCR composition to 1 AU by solving a steady-state, spherically symmetric Fokker-Planck transport equation, which accounts for diffusion, convection, and adiabatic deceleration of cosmic rays entering the heliosphere (Parker, 1965). The transport physics described above enables the temporal and spatial dependence of the GCR transport to be absorbed into a ratio of the diffusion coefficient to the bulk solar wind speed. The functional form of this ratio is given by

$$
\tilde{k}(r, t) \equiv k(r, t) / V_{S W}(r, t)=\left(k_{0} / V_{S W}\right) \beta R\left[1+\left(r / r_{0}\right)^{2}\right] / \Phi(t)
$$


where $V_{\mathrm{SW}}$ is the bulk solar wind speed (nominally set to $400 \mathrm{~km} / \mathrm{s}$ for all time $\mathrm{t}$ ), $r$ is the distance from the sun in AU, $t$ is time in years, $k_{0}$ and $r_{0}$ are constants, $\beta$ is the particle's speed relative to the speed of light, $R$ is the particle's magnetic rigidity in MV, and $\Phi$ is the so-called solar modulation parameter. Thus, the time-dependent behavior of the GCR spectral flux, due to the level of solar activity, is completely embedded in the solar modulation parameter. The solar modulation parameter is physically related to the energy that interstellar nuclei must have in order to overcome the heliospheric potential field, established by the large-scale structure of the IMF, and propagate through the heliosphere to the radius in question. The solar modulation parameter is determined by fitting the solution of the Fokker-Planck equation for a specified GCR nuclei to corresponding spectral flux measurements throughout the solar cycle, as described in the paragraph below (O'Neill, 2006).

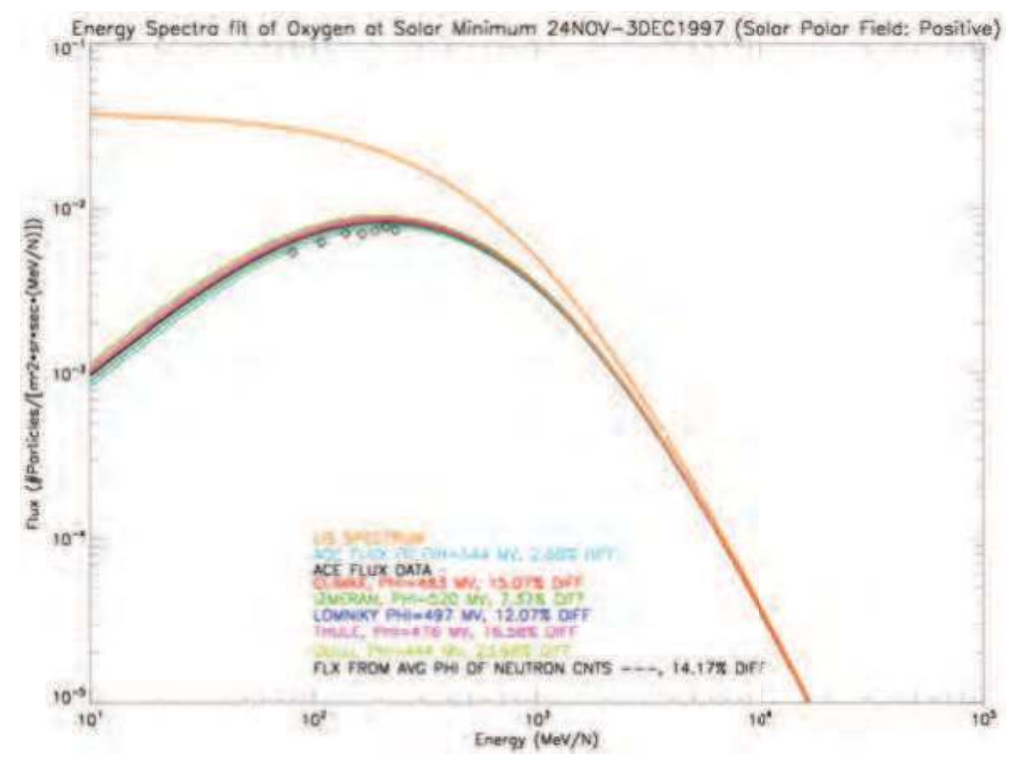

Fig. 6. NAIRAS GCR model spectral oxygen nuclei flux comparisons with ACE/CRIS measurements for solar minimum conditions. The GCR model flux was computed for neutron monitor count rates measured from the four high-latitude sites shown in Figure 4. Also shown are the oxygen spectral flux predicted by the reference Climax-based solar modulations parameter and the GCR model oxygen spectral flux computed using the average solar modulation parameter predicted by each neutron monitor site.

For a fixed parameterization of the LIS, the solar modulation parameter in (1) (i.e., $\Phi(t)$ ) was determined by fitting the solution of the steady-state Fokker-Planck equation for oxygen nuclei to measurements of the corresponding spectral flux. For energies below roughly 1 $\mathrm{GeV}$ (i.e. $\sim 50-500 \mathrm{MeV} /$ nucleon), the measurement data were obtained from the Cosmic Ray Isotope Spectrometer (CRIS) instrument on the NASA/ACE satellite. For higher energies (1-35 GeV), the model was fit to data from the C2 instrument on the NASA High Energy Astrophysical Observatory (HEAO-3) satellite (Engelmann et al., 1990).

It is difficult to distinguish the GCR and solar components for protons and alpha spectra observed by CRIS. Fortunately, Lopate (2004) provided an extensive database of quiescent proton and alpha specta from IMP- 8 measurements. Thus, the proton and alpha spectra in the 
GCR model were fit to IMP-8 data. The high energy proton and alpha spectra were fit to the balloon-borne Isotope Matter-Antimatter Experiment (IMAX) measurements (Menn \& et al., 2000).

Once the solar modulation parameter was derived based on the ACE/CRIS oxygen spectra, as described in the above paragraph, the LIS for the remaining elements (i.e., lithium $(Z=3)$ through nickel $(Z=28)$ ) were similarly determined by fitting the solutions of the Fokker-Planck equation to the CRIS spectral flux measurements. A simple power law form of the differential LIS was assumed,

$$
j_{\text {LIS }}(E)=j_{0} \beta^{\delta}\left(E+E_{0}\right)^{-\gamma}
$$

where $E$ is the particle kinetic energy per nucleon and $E_{0}$ is the rest mass energy per nucleon $(938 \mathrm{MeV} / \mathrm{n})$. The free parameters $\left(\gamma, \delta\right.$, and $\left.j_{0}\right)$ were determined from the fit of the GCR model to the CRIS measurements.
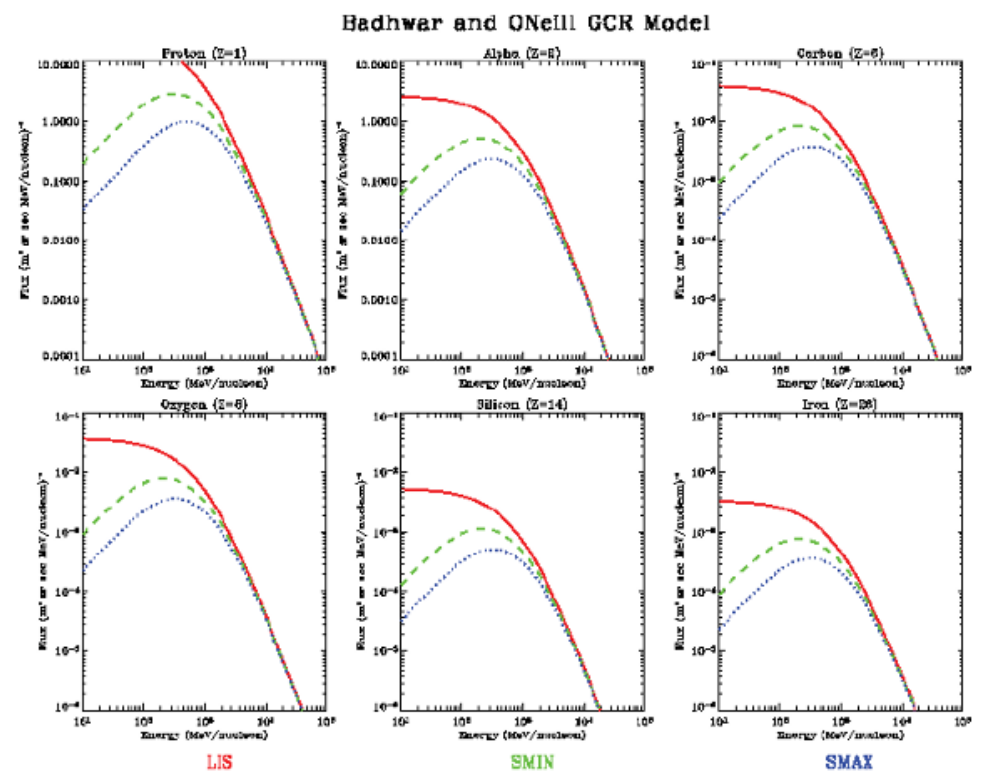

Fig. 7. GCR spectral flux for various nuclei predicted by the Badhwar and O'Neill model for solar cycle 23. The local interstellar spectrum (LIS) is denoted by the red lines. Solar minimum spectra are represented by June 1996 conditions, and are denoted by green lines. Solar maximum spectra are represented by June 2000 conditions, and are denoted by blue lines.

The GCR model was extended beyond the time period of the ACE/CRIS measurements in the following way. First, the solar modulation parameter was alternatively derived from the IMP-8 channel 7 ( $Z>8$, high energy) measurements over three solar cycles from 1973 through 2001, and was calibrated against the solar modulation parameter derived from ACE/CRIS for the period of data overlap (1997.6 to 2001.8). GCR flux comparisons using both sets of solar modulation parameters correlated to within $98.9 \%$. Next, linear fit coefficients were derived between the IMP-8 solar modulation parameter and Climax neutron monitor count rates from 1973-2001. The solar modulation parameter computed using the Climax neutron count rates 
correlated with the solar modulation parameter derived from IMP-8 data within $97 \%$. Linear fits were derived for the three polarity states of the solar polar magnetic field: (1) positive solar cycle (outward field), (2) negative solar cycle (inward field), and (3) transition state (intermediate between positive and negative polarities with a high degree of modulation). The solar modulation parameter derived from Climax neutron count rates has been recently extended from 1958-2009. This extended Climax-based solar modulation parameter provides the reference solar modulation parameter from which to derive a real-time GCR model suitable for integration into the NAIRAS model.

Four neutron monitor sites - Thule, Oulu, Izmiran, and Lomnicky - were chosen to develop the NAIRAS GCR model. These high-latitude sites were chosen to maximize the solar cycle information content contained in the GCR spectrum and embedded in the ground-based neutron count rates. The neutrons detected on the ground are secondary particles produced by nuclear fragmentation reactions between the incoming GCR particles and the atmospheric constituents (Wilson et al., 1991). At high-latitudes the geomagnetic shielding of the incoming GCR particles is low. Thus, the information contained in the ground-level neutron counts on the low- and medium energy region of the GCR spectrum is high. This is highly desirable since this energy range of the GCR spectrum is most modulated by the solar wind and IMF, and thus closely related to the solar activity cycle (Mertens et al., 2008).

The real-time NAIRAS GCR model was developed by cross-correlating the Climax-based solar modulation parameter (denoted $\Phi_{\text {CLIMAX) }}$ ) with the neutron count rates measured at the four high-laitude sites mentioned above. Thus, linear fit coefficients were derived between $\Phi_{\text {CLIMAX }}$ and the neutron data at the four high-latitude sites. Monthly-mean neutron count rates at the four high-latitude sites are shown in Figure 4a. Figure $4 \mathrm{~b}$ shows the solar modulation parameter predicted by the monthly-mean neutron count rates shown in Figure 4 a. For comparison, the reference Climax-based solar modulation parameter ( $\left.\Phi_{\text {CLIMAX }}\right)$ is shown, along with the solar modulation parameter derived from NASA/ACE measurements (denoted $\Phi_{\mathrm{ACE}}$ ).

The heliospheric GCR diffusion coefficient depends on the large-scale structure of the IMF (Parker, 1965). As a result, the diffusion coefficient will depend on the polarity of the Sun's polar magnetic field ( $\mathrm{O}^{\prime}$ Neill, 2006). Consequently, to improve the accuracy of the neutron count rate fits to the reference $\Phi_{\text {CLIMAX, }}$ the data were sorted according to the polarity of the Sun's polar magnetic field, and three sets of fit coefficients were derived: (1) positive solar cycle (outward field), (2) negative solar cycle (inward field), and (3) transition state (intermediate between positive and negative polarities with a high degree of modulation), as described previously for the $\Phi_{\text {CLIMAX }}$ reference solar modulation parameter. Figure 5 shows the solar polar magnetic field data since 1978, which are obtained from measurements taken at the Wilcox Solar Observatory (WSO) located at Stanford University. The solar polar magnetic field data from WSO have been added to the NAIRAS input data stream.

Figure 6 shows a comparison between the NAIRAS GCR model spectral oxygen nuclei flux and measurements taken by ACE/CRIS for solar minimum conditions. Also shown is the oxygen spectral flux predicted by the reference solar modulation parameter ( $\Phi_{\text {CLIMAX }}$ ). Nominal NAIRAS operations uses the average solar modulation parameter determined from the available high-latitude neutron monitor count rate data. Figure 6 shows the nominal NAIRAS GCR oxygen spectral flux determined from all four high-latitude neutron sites (see Figure 4). In this case, the error in the nominal NAIRAS prediction of the incident GCR oxygen flux is comparable to the error of the predicted flux based on the reference solar modulation parameter $(\sim 15 \%)$. 
A comprehensive set of error statics on the real-time NAIRAS GCR model has been compiled. Generally, the error in using the count rates from the high-latitude neutron monitor stations is comparable to the error in the Badhwar and O'Neill GCR model based on the reference solar modulation parameter. All errors are much larger for solar maximum conditions compared to solar minimum conditions. The main reason for this is that the ACE data are more suspect during solar maximum due to the combination of higher noise levels (GCR flux is minimum during solar maximum) and contamination by particles of solar origin.

Figure 7 shows the solar cycle variation in the GCR spectrum for several nuclei. The spectra were computed by the Badhwar and O'Neill model (Mertens et al., 2008; 2007a). The figure shows the LIS spectra and the spectra at solar maximum and solar minimum conditions. The solar cycle modulation of LIS as the GCR nuclei are transported through the heliosphere to 1 AU is clearly evident.

\subsection{Geospace SEP fluence rate specification}

The solar cosmic rays from SEP events are not transported from the Sun to the geospace environment using a model. Rather, in-situ satellite measurements of ion flux are used to constrain analytical representations of the SEP fluence rate spectrum. However, the analytical functions are guided by the state-of-the-art understanding of the origin, acceleration, and transport of energetic particles from the solar atmosphere to the geospace environment via the interplanetary medium.

The current understanding of SEP processes is that the energy spectrum is a result of injected particle seed populations that are stochastically accelerated in a turbulent magnetic field associated with a CME-driven interplanetary shock (Tylka \& Lee, 2006). An analytical expression that represents the differential energy spectrum for this shock acceleration mechanism was given by Ellison \& Ramaty (1985), where the spectrum has the form

$$
\frac{d^{2} J}{d E d \Omega}=C_{a} E^{-\gamma_{a}} \exp \left(-E / E_{0}\right)
$$

The differential energy spectrum on the left hand side of (3) has units of $\left(\mathrm{cm}^{2}-\mathrm{sr}-\mathrm{hr}-\mathrm{MeV} / \mathrm{n}\right)^{-1}$, and the energy $(E)$ has units of $\mathrm{MeV} / \mathrm{n}$ (i.e., $\mathrm{MeV} /$ nucleon). The constant $C_{a}$ is related to the injected seed population far upstream of the shock. The power-law energy dependence of the spectrum is due to shock acceleration of the seed population by random first-order Fermi acceleration (scattering) events in a turbulent magnetic field, with the power index $\left(\gamma_{a}\right)$ related to the shock compression ratio. The exponential turnover in (3) represents high-energy limits to the the acceleration mechanism, such as escape from the shock region. Using the above analytical form, the three parameters $\left(C_{a}, \gamma_{a}\right.$, and $\left.E_{0}\right)$ can be determined by fitting (3) to ion flux measurements.

Recently, Mewaldt et al. (2005) found that the Ellison-Ramaty spectral form failed to fit NOAA-GOES ion flux measurements at the highest energy channels during the Halloween 2003 SEP events. To circumvent this deficiency, Mewaldt et al. proposed using a double power-law spectrum. The low-energy spectrum is assumed to follow the Ellison-Ramaty form. The high-energy spectrum is assumed to have a power-law energy dependence with a different power index, such that

$$
\frac{d^{2} J}{d E d \Omega}=C_{b} E^{-\gamma_{b}}
$$


The power-law expressions in (3)-(4) can be merged into one continuous spectrum by requiring that the differential energy spectra in (3)-(4) and their first derivatives are continuous at the merge energy. The result is given by the expression below:

$$
\begin{aligned}
\frac{d^{2} J}{d E d \Omega} & =C E^{-\gamma_{a}} \exp \left(-E / E_{0}\right), \quad E \leq\left(\gamma_{b}-\gamma_{a}\right) E_{0} \\
& =C E^{-\gamma_{b}}\left\{\left[\left(\gamma_{b}-\gamma_{a}\right) E_{0}\right]^{\left(\gamma_{b}-\gamma_{a}\right)} \exp \left(\gamma_{b}-\gamma_{a}\right)\right\}, \quad E>\left(\gamma_{b}-\gamma_{a}\right) E_{0} .
\end{aligned}
$$

Physically, the double power-law spectrum in (5)-(6) represents SEP sources from two different injected seed populations. For example, the low-energy spectrum, with $\gamma_{a}$ power index and the e-folding energy $E_{0}$, is likely associated with solar corona (solar wind) seed populations while the high-energy spectrum, with $\gamma_{b}$ power index, is likely associated with flare suprathermal seed populations (Tylka et al., 2005).

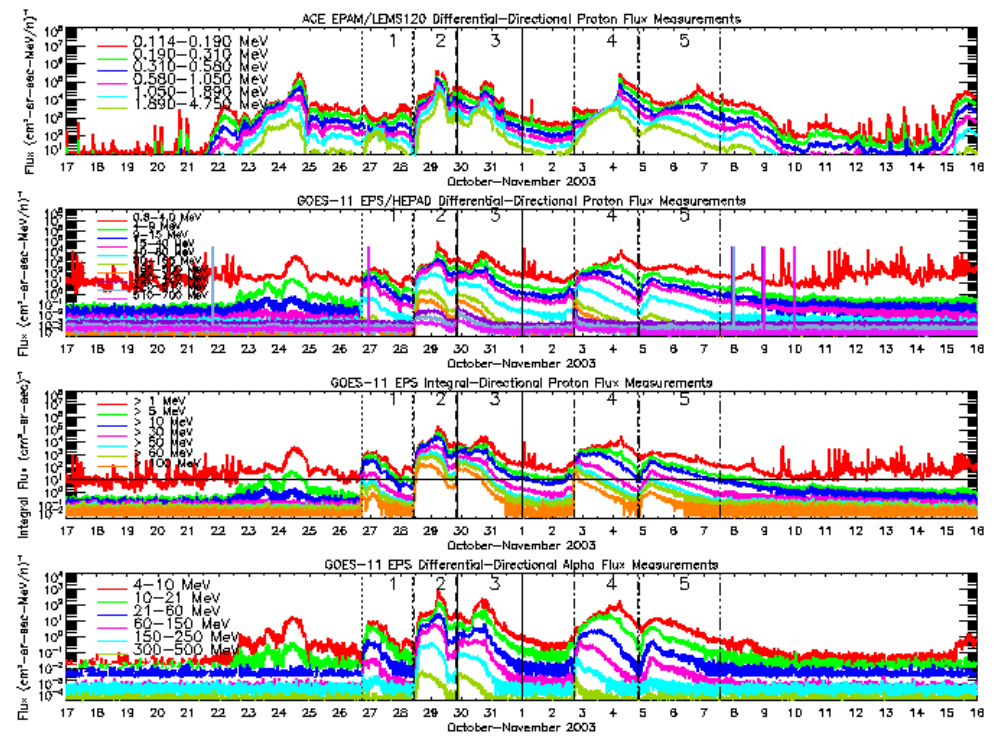

Fig. 8. Proton and alpha flux measurements used to derive the SEP fluence spectra. Row 1: ACE EPAM/LEMS120 differential-directional proton flux measurements. Row 2: GOES-11 EPS and HEPAD differential-directional proton flux measurements. Row 3: GOES-11 EPS integral-directional proton flux measurements. Row 4: GOES-11 EPS differential-directional alpha flux measurements. The different styled vertical lines bound the five SEP events during the Halloween 2003 solar-geomagnetic storm period, which are numbered in all panels. The horizontal line in Row 3 indicates the SEP threshold for the $>10 \mathrm{MeV}$ integral proton flux channel.

Another widely used analytical representation of a SEP energy spectrum is the so-called Weibull distribution (Townsend et al., 2006; 2003). The Weibull distribution has been successful at fitting satellite ion flux measurements, and the differential energy spectrum is given by

$$
\frac{d^{2} J}{d E d \Omega}=C k \alpha E^{\alpha-1} \exp \left(-k E^{\alpha}\right)
$$


Notice that the Weibull distribution has an analytical form similar to the Ellison-Ramaty distribution in (3). The exponential energy dependence in the Weibull differential energy spectrum could be due to dissipation of the high-energy SEP ions by scattering from self-generated waves. However, further investigation is required to determine if this type of physical process is related to the Weibull distribution (Xapsos et al., 2000).

The NAIRAS model fits four analytical SEP spectral fluence rate functions to the satellite ion flux measurements. The analytical forms that are fit to the measurements are: (1) single power-law in (4), (2) Ellison-Ramaty in (3), (3) double power-law in (5)-(6), and (4) Weibull in (7). The free parameters for each analytical differential energy distribution are derived by a non-linear least-square fit to differential-directional ion flux measurements. The spectral fitting algorithm uses a Marquardt-Levenberg iteration technique (Brandt, 1999). The analytical form that yields the minimum chi-square residual in the fit to the ion flux measurements is the SEP spectral fluence rate distribution used in all subsequent model simulations.

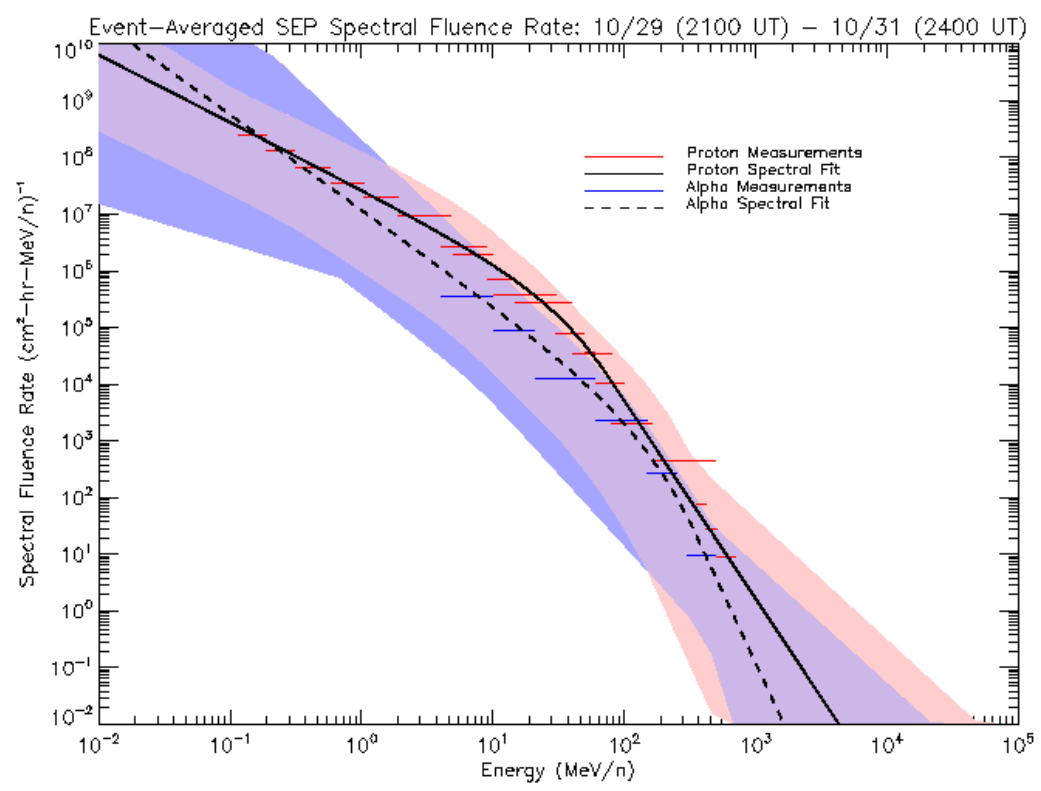

Fig. 9. Event-Averaged SEP spectral fluence rates for Halloween 2003 SEP event 3 [10/29/2003 (2100 UT) - 10/31/2003 (2400 UT)]. The shaded regions show the range of

1 -hour averaged ion flux measurements and fitted spectra in the time interval of event 3 . The peach shaded regions corresponds to proton flux measurements. The blue shaded regions corresponds to the alpha flux measurements.

NAIRAS utilizes available real-time measurements of proton and alpha differential-directional particle flux $\left(\mathrm{cm}^{2} \text {-sr-sec-MeV/n }\right)^{-1}$ for the SEP spectral fitting described above. SEP spectral fluence rates $\left(\mathrm{cm}^{2}-\mathrm{hr}-\mathrm{MeV} / \mathrm{n}\right)$ incident on Earth's magnetosphere are obtained by time-averaging the particle flux measurements in 1-hr time bins and projecting the incident flux onto the vertical direction assuming an isotropic angular distribution for the solar ions. Low-energy proton data are obtained from the 
Electron, Proton, and Alpha Monitor (EPAM) instrument onboard the NASA/ACE satellite (Gold \& et al., 1998). EPAM is composed of five telescopes and the LEMS120 (Low-Energy Magnetic Spectrometer) detector in used in the SEP spectral ion fit, which measures ions at 120 degrees from the spacecraft axis. LEMS120 is the EPAM low-energy ion data available in real-time, for reasons described by Haggerty \& Gold (2006). The other proton channels used in the SEP spectral fitting algorithm are obtained from NOAA/GOES Space Environment Monitor (SEM) measurements. The Energetic Particle Sensor (EPS) and the High Energy Proton and Alpha Detector (HEPAD) sensors on GOES/SEM measure differential-directional proton flux (Onsager \& et al., 1996). Additional differential-directional proton flux measurement channels are generated by taking differences between the EPS integral proton flux channels. The channels used to derive SEP alpha spectral fluence rates are also obtained from EPS measurements. Five-minute averaged ACE and GOES data are used to derive the incident SEP spectral fluence rates.

Figure 8 shows the time variation of the proton and alpha flux measurements used to derive incident SEP spectral fluence rates for the Halloween 2003 storm period. The top panel displays the ACE low-energy proton flux measurements. The next two panels show the GOES-11 EPS and HEPAD proton flux spectra, and the integral proton flux measurements, respectively. By definition, a SEP event occurs when the $>10 \mathrm{MeV}$ integral proton flux exceeds 10 proton flux units ( $\mathrm{pfu} \equiv \mathrm{cm}^{-2} \mathrm{sr}^{-1} \mathrm{sec}^{-1}$ ) in three consecutive 5 -minute periods (NOAA, 2009). The SEP event threshold is denoted by the horizontal line on the integral proton flux panel. There are a total of five SEP events during the Halloween 2003 storm period, which are denoted by the vertical lines in all panels in Figure 8 . These events were associated with many simultaneous, complex phenomena such as solar flares, coronal mass ejections (CME), interplanetary shocks, and solar cosmic ray ground level events (GLE) (Gopalswamy et al., 2005). Different line styles are used to bound each of the five events, and the event number is shown between the vertical lines. Note that the onset of event 3 doesn't follow the conventional SEP threshold definition. It is clear from the integral proton flux that two events overlap: event 3 arrives before event 2 decreases below the SEP threshold level. However, there is an important distinguishing feature between the two events. That is, the beginning of our definition of event 3 is accompanied by a sudden increase in high-energy protons associated with the arriving SEP event, as noted by the sudden increase in the $510-700 \mathrm{MeV}$ differential-directional proton flux measurements in Figure 8. Partitioning the simultaneous SEP events 2 and 3 into separate events is useful for the aircraft radiation exposure analysis discussed in section 4 , since the high-energy portion of the differential-directional proton flux distribution penetrates deeper in the atmosphere.

In section 4, atmospheric ionizing radiation exposure during SEP event 3 [10/29 (2100 UT) - 10/31 (2400)] will be analyzed since the associated interaction between the arriving CME-driven interplanetary shock and Earth's magnetosphere caused the largest geomagnetic effects during the Halloween 2003 storm period, which is the focus of the case study in section 4.3. In order to isolate the geomagnetic effects, the event-averaged SEP spectral fluence rates is derived and shown in Figure 9. The horizontal lines in Figure 9 are the event-averaged differential ion fluence rate measurements. The width of the horizontal lines correspond to the energy width of the measurement channels. The black lines are the proton and alpha spectral fluence rates derived using the double power-law spectrum and fitting technique describe above. The shaded regions show the range of 1-hour averaged ion spectral fluence rates in the time interval of event 3 . The peach colored region corresponds to the range of proton energy spectra and the blue colored region corresponds to the range of alpha energy spectra. 


\subsection{Magnetospheric cosmic ray transport}

Lower energy cosmic rays are effectively attenuated by the geomagnetic field (internal field plus magnetospheric contributions) as these charges particles are transported through the magnetosphere and into the neutral atmosphere. The geomagnetic field provides a form of momentum shielding, or attenuation, by deflecting the lower-energy charged particles back out to space via the Lorentz force. This spectral filtering effect is quantified by a canonical variable, in the mathematical sense, called the geomagnetic cutoff rigidity. Once the cutoff rigidity is known, the minimum access energy to the neutral atmosphere is determined for each incident charged particle through the relativistic energy equation (Mertens et al., 2010a).

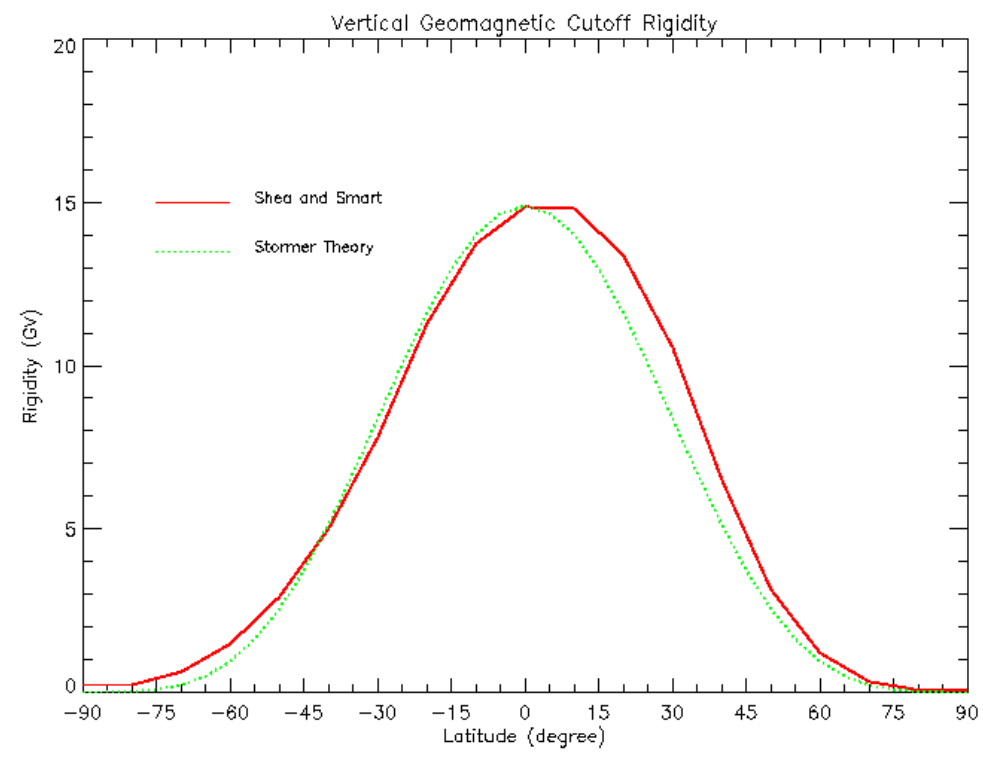

Fig. 10. Zonal-averaged vertical geomagnetic cutoff rigidity. The solid red line corresponds to the quiescent cutoff rigidities computed from particle trajectories and the IGRF model. The green dotted line is the analytical solution for the vertical cutoff rigidity using Störmer Theory.

The utility of the geomagnetic cutoff rigidity quantity is motivated by considering the motion of a charged particle in a magnetic field. The particle motion is determined by solving Newton's equation of motion for a charged particle subject to the Lorentz force. For a positively charged particle, the equation of motion is

$$
\frac{d \mathbf{p}}{d t}=\frac{Z e}{c} \mathbf{v} \times \mathbf{B}
$$

in cgs units. The bold-faced quantities are vectors and $x$ designates the vector cross product. The charged particle momentum and velocity are $\mathbf{p}$ and $\mathbf{v}$, respectively, and $\mathbf{B}$ is the magnetic field strength. The magnitude of the charge of an electron is denoted $e$ and $Z$ is the number of electron charge units. The equation of motion in (8) can be written, equivalently, as

$$
\frac{R}{B} \frac{d \hat{\mathbf{v}}}{d t}=\hat{\mathbf{v}} \times \hat{\mathbf{B}}
$$


where the^symbol denotes units vectors and

$$
R \equiv \frac{p c}{Z e}
$$

is defined as the rigidity. The canonical aspect of the rigidity is evident in the above equation. For a given magnetic field strength(B), charged particles with the same rigidity follow identical trajectories.

Vertical Geomagnetic Cutoff Rigidity: IGRF 1996
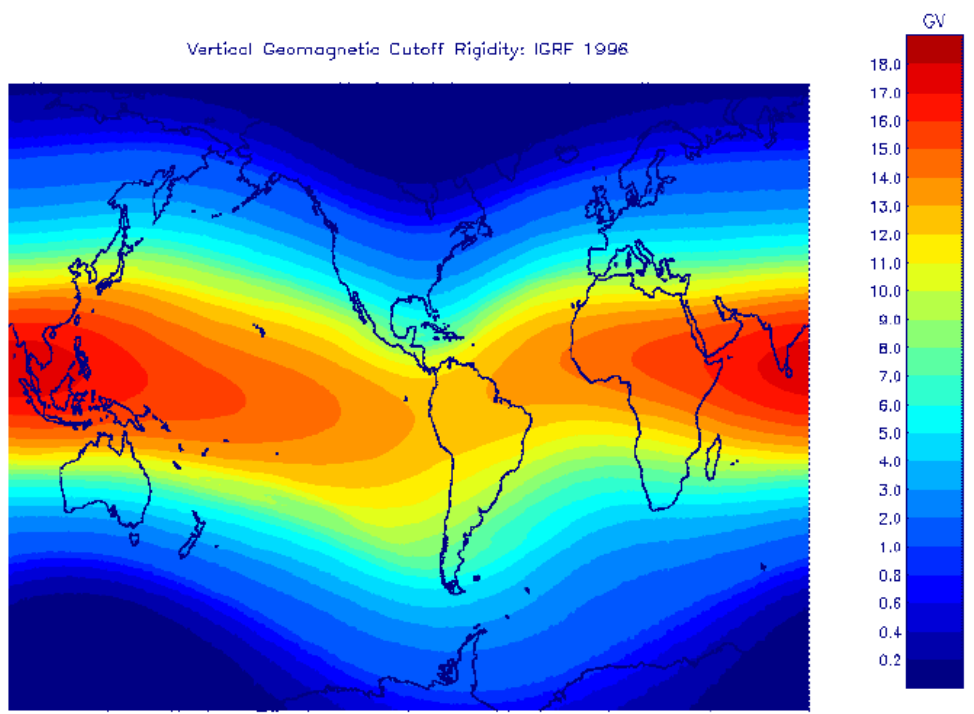

Fig. 11. Global grid of quiescent vertical geomagnetic cutoff rigidities (GV) calculated from charged particle trajectory simulations using the IGRF model for the 1996 epoch (solar cycle 23 minimum).

Motions of charged particles in a pure magnetic dipole field were examined by Störmer (1965). Because of the azimuthal symmetry in a pure dipole field, the azimuthal angular momentum is a conserved quantity. A main feature of Störmer theory is that regions of bounded and unbounded motion can be derived analytically from the integral of motion found from the conservation of azimuthal angular momentum (Störmer, 1965; VanAllen, 1968). It can be shown that the minimum rigidity that a vertically arriving particle must have in order to reach an altitude $z$ above the Earth's surface is

$$
R_{v c}=\frac{\mathcal{M}}{\left(R_{e}+z\right)^{2}} \cos ^{4} \lambda_{m} \approx 15 \cos ^{4} \lambda_{m}(\mathrm{GV}) .
$$

In the above equation, $R_{v c}$ designates the vertical geomagnetic cutoff rigidity, $\mathcal{M}$ is the Earth's magnetic dipole moment, $R_{e}$ is the average radius of the Earth, and $\lambda_{m}$ denotes magnetic latitude. Therefore, vertically arriving charged particles with energies $(E)$ less than the cutoff energy $\left(E_{v c}\right)$ will be deflected by the Lorentz force and not reach altitude $z$. The cutoff energy for each charged particle of charge $Z$ and mass number $A$ is determined from the canonical 
cutoff rigidity through the relativistic energy equation, such that

$$
E_{v c}=\left[\sqrt{R_{v c}^{2}\left(Z / A \cdot \mathrm{amu} \cdot c^{2}\right)^{2}+1}-1\right] \cdot \mathrm{amu} \cdot c^{2},
$$

where $E$ is kinetic energy per nucleon $(\mathrm{MeV} / \mathrm{n}), R_{v c}$ is vertical geomagnetic cutoff rigidity $(\mathrm{MV}), c$ is the speed of light in vacuum, and amu $=931.5 \mathrm{MeV} / \mathrm{c}^{2}$ (atomic mass unit). Thus, the geomagnetic field has the effect of filtering out lower-energy charged particles as they are transported through the magnetosphere and into the neutral atmosphere.

The vertical cutoff rigidity at the Earth's surface derived from Störmer theory is plotted versus geographic latitude in Figure 10. The maximum cutoff rigidity is at the equator since a vertically arriving charged particle is perpendicular to the dipole magnetic field lines at the equator. The affect of the vector cross product in the Lorentz force in (8) is that charged particle motions perpendicular to magnetic field lines will experience the maximum deflection while particle motions parallel to the magnetic field will experience no deflecting force whatsoever. Figure 10 illustrates that a vertically arriving proton at the equator must have a kinetic energy of $\sim 15 \mathrm{GeV}$ to arrive near the surface of the Earth. In the polar regions, vertically arriving charged particles are parallel to the magnetic field lines. Therefore, the cutoff rigidity is zero; particles of all energies can arrive at the Earth's surface in this case.
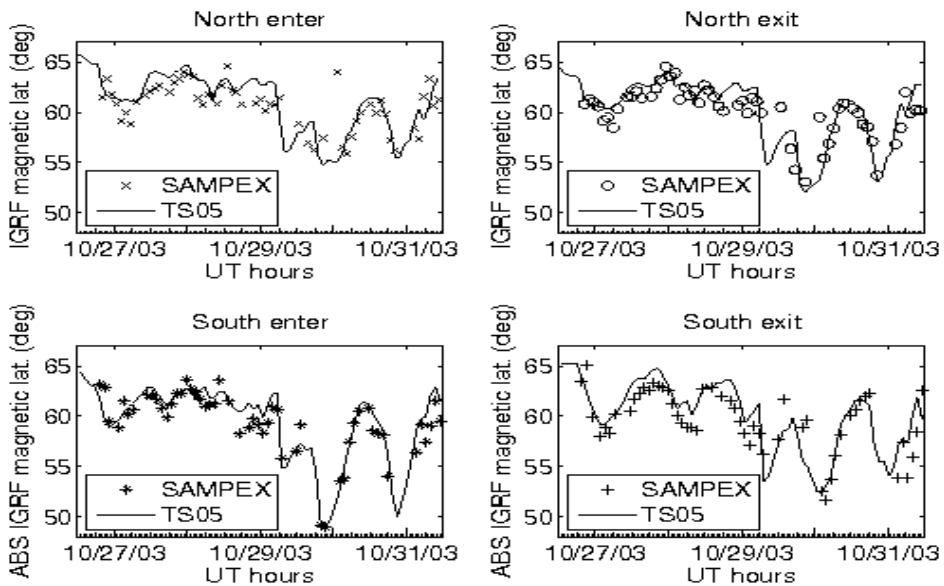

Fig. 12. The four panels show comparisons between cutoff latitudes determined in the TS05 geomagnetic field model and cutoff latitudes extracted from SAMPEX/PET energetic particle data during 28-31 October (Halloween) 2003 storms. Cutoff latitudes as SAMPEX enters and exists the north and south polar cap regions are shown separately.

The Earth's geomagnetic field is not a pure dipole field. On the contrary, the internal geomagnetic field is comprised of dipolar and non-dipolar contributions (Langlais \& Mandea, 2000). The dipole moment is off-center and tilted with respect to the rotational axis. Furthermore, the geomagnetic field is distorted at large radial distances $\left(r \geq 4 R_{e}\right)$ by its interaction with the solar wind. A balance between the solar wind dynamic pressure and the magnetic field pressure, from the internal geomagnetic field, is established by inducing five magnetospheric current systems (Tsyganenko, 1989; 2002). These current systems generate their own magnetic fields which add vectorally to the internal geomagnetic field. 
The complexities of the actual internal geomagnetic field, with dipolar and non-dipolar contributions, and the magnetospheric magnetic field contributions prohibit an analytical solution for the vertical geomagnetic cutoff rigidity. Numerical methods must be employed, as described below.
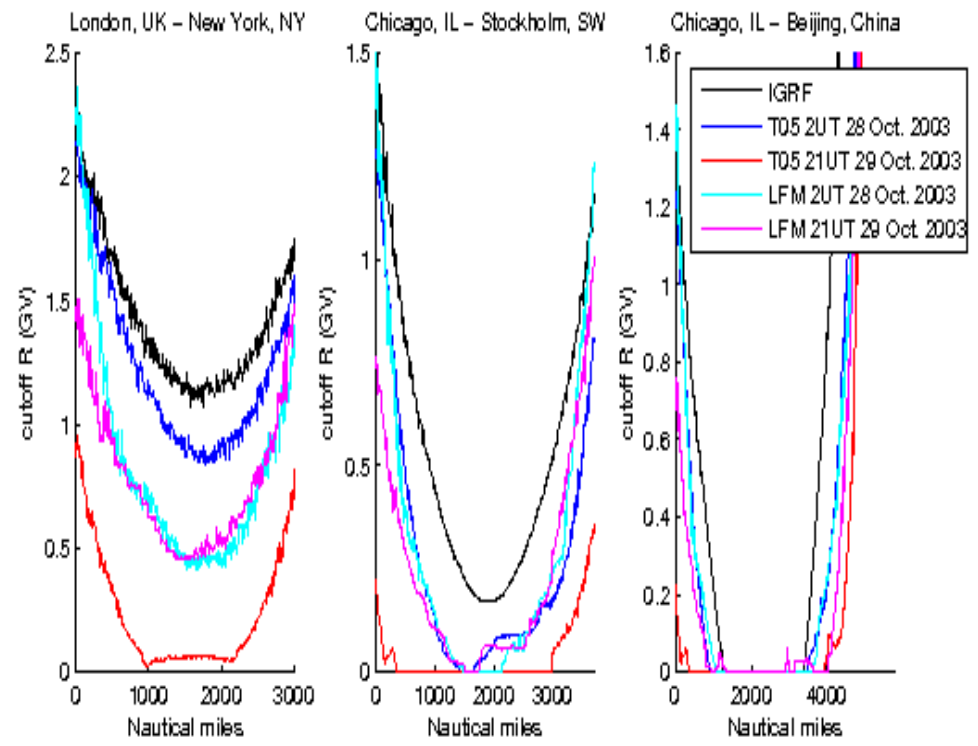

Fig. 13. Geomagnetic cutoff rigidities along three high-latitude commercial flight routes computed in three different magnetic field models: IGRF, TS05, and LFM MHD. The October 28 (0200 UT) calculations represent a magnetically quiet time, while the October 29 (2100 UT) calculations occur during a geomagnetic storm.

The internal geomagnetic field is specified by the International Geomagnetic Reference Field (IGRF) model (Langlais \& Mandea, 2000). In the IGRF, the internal field is represented in terms of a magnetic potential function $\left(\Phi_{M}(r, \theta, \phi, t)\right)$. Outside of the internal source region, i.e, for $r \geq R_{e}$, the magnetic potential function must be a solution of the Laplace equation. In spherical coordinates, this solution is expressed in the following form:

$$
\Phi_{M}(r, \theta, \phi, t)=R_{e} \sum_{n=1}^{N} \sum_{m=0}^{n}\left(\frac{R_{e}}{r}\right)^{n+1}\left[g_{n}^{m}(t) \cos m \phi+h_{n}^{m}(t) \sin m \phi\right] P_{n}^{m}(\cos \theta) .
$$

In the above equation, $r$ denotes the radial distance from the center of the Earth, $\theta$ and $\phi$ denote the geocentric colatitude and longitude at a given location. Schmidt-normalized associated Legendre functions of degree $n$ and order $m$ are denoted by $P_{n}^{m}(\cos \theta)$, and $g_{n}^{m}$ and $h_{n}^{m}$ denote the Gauss coefficients. The magnetic field components are given by the gradient of the potential function, such that

$$
B_{r}=\frac{\partial \Phi_{M}}{\partial r}, B_{\theta}=\frac{1}{r} \frac{\partial \Phi_{M}}{\partial \theta}, B_{\phi}=\frac{1}{r \sin \theta} \frac{\partial \Phi_{M}}{\partial \phi} .
$$


The Gauss coefficients are derived from a global set of magnetic field measurements, using the method of least-squares, and updated every five years (Langlais \& Mandea, 2000). Secular variations in the Gauss coefficients are also derived from magnetic field measurements so that derivatives of the Gauss coefficients can be computed. In this way, the temporal dependence of the internal geomagnetic field is represented by

$$
\begin{aligned}
& g_{n}^{m}(t)=g_{n}^{m}\left(T_{0}\right)+\dot{g}_{n}^{m}\left(t-T_{0}\right) \\
& h_{n}^{m}(t)=h_{n}^{m}\left(T_{0}\right)+\dot{h}_{n}^{m}\left(t-T_{0}\right)
\end{aligned}
$$

where $\dot{g}_{n}^{m}$ and $\dot{h}_{n}^{m}$ are first-order derivatives of the Gauss coefficients. The epoch of the IGRF model is denoted by $T_{0}$ and $t$ is such that $T_{0} \leq t \leq T_{0}+5$, where time is expressed in decimal years.

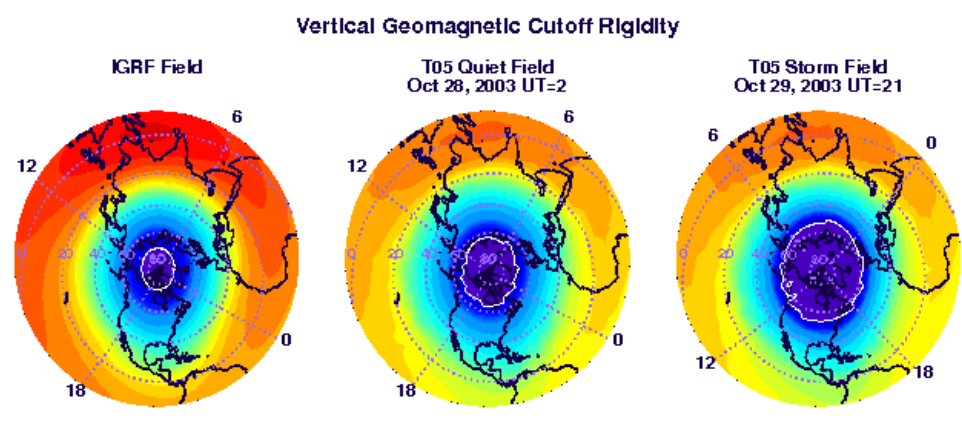

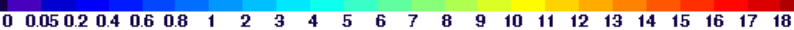

GV

Fig. 14. Simulated vertical geomagnetic cutoff rigidity shown over the northern hemisphere in October 2003. The cutoff rigidities in the left column were calculated using the IGRF model. The cutoffs in the middle column were calculated using the TS05 model during a geomagnetically quiet period. Cutoff rigidities in the right column were calculated using the T05 model during the largest geomagnetically disturbed period of SEP event 3 . Also shown are the magnetic latitude circles and the meridians at $0,6,12$, and 18 magnetic local time.

The vertical cutoff rigidities in a realistic geomagnetic field are determined by numerical solutions of charged particle trajectories in the magnetic field using the techniques advanced by Smart \& Shea $(1994 ; 2005)$. Figure 11 shows the vertical cutoff rigidities at $20 \mathrm{~km}$ in the internal IGRF field. The longitudinal variations in the cutoff rigidity are due to a combination of geocentric offset and relative tilt of the magnetic dipole, with respect to the rotational axis, and the non-dipolar contributions to the internal geomagnetic field. A zonal-average of the IGRF cutoff rigidities in Figure 11 are compared to the analytical Störmer theory in Figure 10. The simple Störmer theory represents the latitudinal behavior of the vertical cutoff rigidity quite well. The displacement of the Störmer theory cutoffs in Figure 10 relative to the numerical solutions of the cutoffs in the IGRF field is due to the fact that the true dipole contribution to the internal geomagnetic field is off-centered and tilted with respect to the rotational axis.

NAIRAS real-time geomagnetic cutoff rigidities are computed from numerical solutions of charged particle trajectories in a dynamically varying geomagnetic field that includes both the internal magnetic field and the magnetospheric magnetic field contributions (Kress et al., 


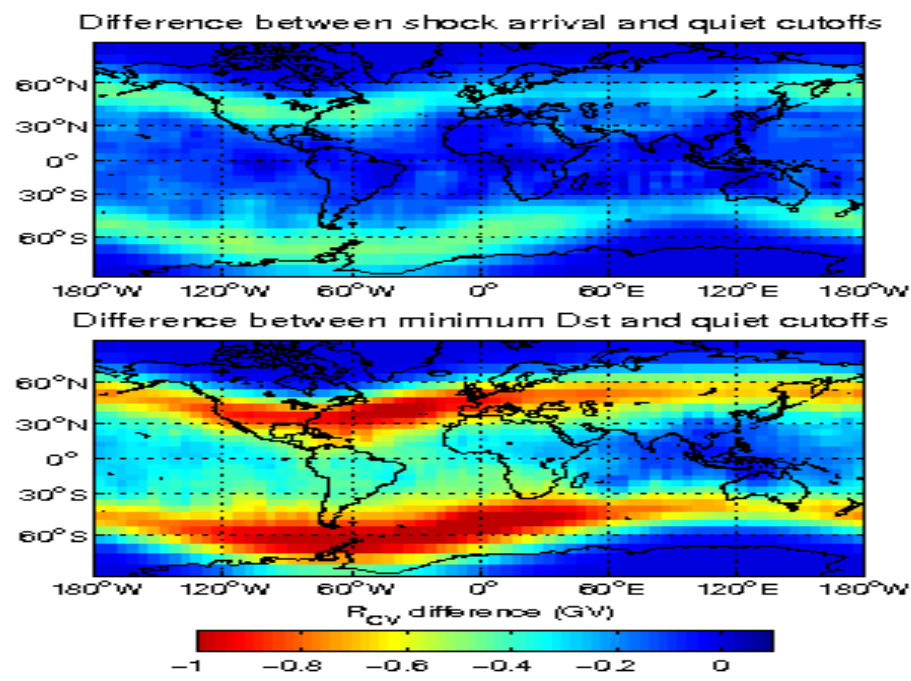

Fig. 15. Storm-quiet geomagnetic cutoff rigidities differences calculated using the TS05 magnetospheric magnetic field model. The magnetically quiet time cutoffs were calculated on October 28 (0200 UT), 2003. The storm cutoffs in the top panel were computed when the interplanetary shock arrived on October 29 (0612 UT), 2003. The storm cutoffs in the bottom panel were computed at the maximum build-up of the ring current on October 29 (2100 UT), 2003.

2010; Mertens et al., 2010a; 2009). The cutoff rigidity code was developed by the Center for Integrated Space Weather Modeling (CISM) at Dartmouth College. The CISM-Dartmouth geomagnetic cutoff model can be run using several different empirical, semi-physics-based, and physics-based models (Kress et al., 2004). In particular, the specification of the geomagnetic field due to Earth's internal field source is provided by the IGRF model [Langlais and Mandea, 2000], as discussed above. The real-time dynamical response of the magnetospheric magnetic field to solar wind conditions and IMF can be provided by the semi-physics-based TS05 model (Tsyganenko \& Sitnov, 2005), or by the Lyon-Feder-Mobarry (LFM) global MHD (magnetohydrodynamic) simulation code (Fedder \& Mobarry, 2004). Routines were developed and tested to couple the geomagnetic cutoff model with the different magnetic field models.

The LFM MHD code may be run as a stand alone model or coupled with other geospace models currently under development within CISM. For example, the LFM magnetospheric magnetic fields may be coupled with the Thermosphere-Ionosphere Nested Grid (TING) model (Wang et al., 2004) and/or with the Rice Convection Model (RCM) (Toffoletto et al., 2004), which models the ring current. The semi-physics-based TS05 model provides more accurate cutoff rigidities than the stand alone LFM MHD model, as determined by comparisons with satellite observations during a Halloween 2003 geomagnetic storm. Figure 12 shows comparisons between cutoff latitudes for $\sim 20 \mathrm{MeV}$ protons computed using the TS05 model and measured by the Proton Electron Telescope (PET) instrument on the Solar Anomalous and Magnetospheric Particle Explorer (SAMPEX) satellite (Baker et al., 1993). 
The agreement between SAMPEX/PET measurements and the TS05 model in Figure 12 are quite good. The reason the physics-based LFM MHD model doesn't calculate cutoffs as accurate as the semi-physics-based TS05 model is mainly due to the lack of a full kinetic description of the ring current in the MHD model, which typically causes the LFM fields to be too high. This is evident in Figure 13, which compares geomagnetic cutoff rigidities calculated along three representative high-latitude commercial flight routes from the IGRF, TS05, and LFM MHD magnetic field models. The small differences in cutoffs using the LFM MHD model between quiet and geomagnetic storm conditions is indicative of an inadequate modeling of the ring current build-up during the geomagnetic storm.

It is anticipated that the fully coupled LFM-RCM-TING model currently under development will significantly improve the simulations of cutoff rigidities compared to the stand alone LFM MHD model. Furthermore, the physics-based LFM-RCM-TING model will be able to incorporate short time-scale dynamics not included in semi-physics-based (empirical) magnetospheric magnetic field models. When the code development within CISM reaches sufficient maturity, the influence of short time-scale magnetospheric dynamics on the atmospheric ionizing radiation field using the fully coupled LFM-RCM-TING model will be assessed. For the present work, the simulated real-time geomagnetic cutoff rigidities are calculated using the TS05 model, and using the IGRF model for comparison.

Figure 14 shows the vertical cutoff rigidity over the northern hemisphere for three different models of the geomagnetic field during the Halloween 2003 storm period. The left column is cutoff rigidity computed using the IGRF field. Since total flight-path exposure at aviation altitudes do not change significantly $(<\sim 1 \%)$ for cutoffs less than $0.05 \mathrm{GV}$, the cutoffs are set to zero at geographic locations poleward of the $0.05 \mathrm{GV}$ contour (see the bold-white 0.05 GV color contour in Figure 14). The middle column in Figure 14 shows the cutoff rigidities computed using the TS05 field under geomagnetically quiet conditions, October 28 (0200 UT), prior to the onset of the Halloween 2003 SEP event 3 (see section 3.2). One can see that even during magnetically quiet conditions, the cutoff rigidities predicted from the TS05 field are lower than predicted from the IGRF field, and the polar cap region (i.e., inside the bold-white 0.05 GV contour in Figure 14) is expanded to lower latitudes. A weaker field predicted by the TS05 model, compared to IGRF, is due in part to the diamagnetic effect of the magnetospheric ring current included in the TS05 model. Lower cutoff rigidities correspond to less momentum shielding and higher radiation exposure levels. The right column in Figure 14 shows the cutoff rigidities during peak geomagnetic storm conditions, October 29 (2100 UT), during SEP event 3. The cutoffs are lower at all latitudes compared to the two previous simulations, and the polar cap region has expanded to much lower latitudes than during the magnetically quiet period. These geomagnetic effects are discussed in more detail in section 4.3.

The difference in cutoffs between storm and quiet conditions is shown in Figure 15. The cutoffs were calculated using the TS05 model. The magnetically quiet period is the same as above, October 28 (0200 UT), 2003. The top panel shows the cutoff difference between the magnetically quiet time and the arrival of an interplanetary shock at the magnetosphere on October 29 (0612 UT), 2003. The bottom panel shows the storm-quiet cutoff difference when the Disturbed Storm Time (Dst) index is near its minimum on October 29 (2100 UT), 2003. The cutoffs are most suppressed at mid-latitudes during the night. The storm-quiet cutoff difference can be as much as $\sim 1 \mathrm{GV}$, which has a significant effect on radiation exposure.

An important aspect of these model studies is our assessment of the impact of the changes in cutoff rigidity due to the magnetospheric field effect on atmospheric radiation exposure, and the identification of the need for accurate and computationally efficient geomagnetic 
cutoff rigidity models with solar wind-magnetospheric dynamical responses included. The $\sim 1 \mathrm{GV}$ suppression in cutoff at mid-latitudes during a geomagnetic storm means that high-level SEP radiation exposure normally confined to the polar cap region will be extended to mid-latitudes. More details of these findings are included in section 4.3.

\subsection{Atmospheric cosmic ray transport}

The transport of cosmic rays through the neutral atmosphere is described by a coupled system of linear, steady-state Boltzmann transport equations, which can be derived on the basis of conservations principles (Wilson et al., 1991). The transport equation for the directional fluence $\phi_{j}(\mathbf{x}, \Omega, E)$ of particle type $j$ is given by (Mertens et al., 2008; 2007a)

$$
\mathbf{\Omega} \bullet \nabla \Phi_{j}(\mathbf{x}, \mathbf{\Omega}, E)=\sum_{k} \iint \sigma_{j k}\left(\mathbf{\Omega}, \mathbf{\Omega}^{\prime}, E, E^{\prime}\right) \Phi_{k}\left(\mathbf{x}, \mathbf{\Omega}^{\prime}, E^{\prime}\right) d \mathbf{\Omega}^{\prime} d E^{\prime}-\sigma_{j}(E) \Phi_{j}(\mathbf{x}, \mathbf{\Omega}, E)
$$

where $\sigma_{j}(E)$ and $\sigma_{j k}\left(\Omega, \Omega^{\prime}, E, E^{\prime}\right)$ are the projectile-target macroscopic interaction cross sections. The $\sigma_{j k}\left(\Omega, \Omega^{\prime}, E, E^{\prime}\right)$ are double-differential particle production cross sections that represent all processes by which type $k$ particles moving in direction $\Omega^{\prime}$ with energy $E^{\prime}$ produce a particle of type $j$ moving in direction $\Omega$ with energy $E$, including radioactive decay processes. The total interaction cross section $\sigma_{j}(E)$ for each incident particle type $j$ is

$$
\sigma_{j}(E)=\sigma_{j, a t}(E)+\sigma_{j, e l}(E)+\sigma_{j, r}(E),
$$

where the first term refers to projectile collisions with atomic electrons of the target medium, the second term refers to elastic ion-nucleus scattering, and the third term contains all relevant nuclear reactions. The corresponding differential cross sections are similarly ordered.

Consider the transport of cosmic ray ions through the atmosphere. In this case, the second term in (18) represents elastic ion-nucleus Coulomb scattering between the incident ions and the atoms that comprise the neutral atmosphere. Figure 16 shows the characteristic elastic scattering length versus kinetic energy of various ions colliding with the neutral atmosphere (Mertens et al., 2008). Ion-nucleus scattering becomes important in the atmosphere only at low energies. For example, the length of the Earth's atmosphere in units of areal density is $\sim 1000 \mathrm{~g} / \mathrm{cm}^{2}$. Thus, Figure 16 shows that cosmic ray ions will not elastically scatter off an atmospheric nucleus before reaching the surface unless the ion kinetic energy is well below $1 \mathrm{MeV} / \mathrm{amu}$. However, ions with kinetic energy less than $1 \mathrm{MeV} / \mathrm{amu}$ are stopped via ionization and/or atomic excitation energy loss processes at high altitudes before a scattering event can take place (see Figure 18). Multiple Coulomb scattering and coupling with ionization energy loss become important factors in the transport of ions within living tissue (Mertens et al., 2010b; 2007b), which are related to the degree of biological damage inflicted on sensitive components within the living cell. However, for cosmic ray transport through the atmosphere, the ion-nucleus scattering term in (18) can be neglected to a good approximation.

The principle mechanism for atomic interactions between the cosmic ray ions and the target medium is ionization and/or atomic excitation. This process is represented by the first term in (18). The result of this interaction is the transfer of energy from the projectile ions to the atomic electrons of the target medium via the Coulomb impulse force. Since the projectile ion mass is much greater than the electron mass, the ion travels essentially in a straight line as it looses energy through ionization of the target medium. The ionization and atomic excitation 


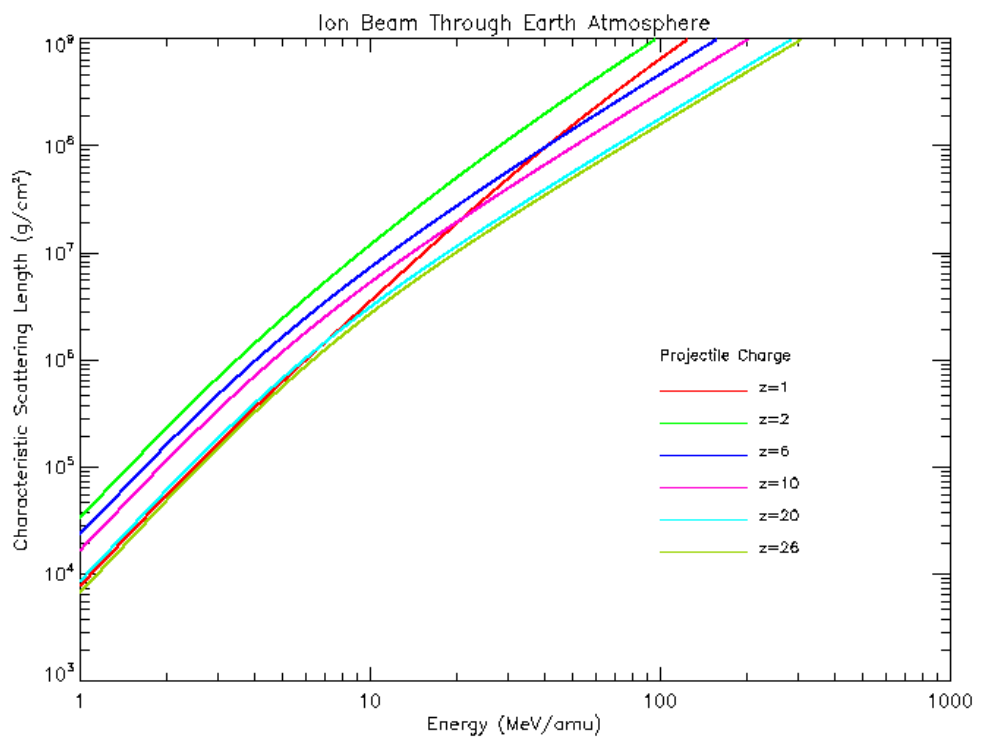

Fig. 16. Characteristic scattering length for ion beam transport through the Earth's atmosphere. The charge number $(\mathrm{z})$ of the ion is specified in the legend.

energies, as well as the energies of ejected orbital electrons, are usually small in comparison to the incident ion kinetic energy. As a result, the ionization energy loss processes by which the projectile ions transfer energy to the target bound and/or ejected orbital electrons can be considered continuous. Because of this so-called continuous slowing down approximation (CSDA), the energy $d E$ which is lost by the incident ion and transferred to the orbital electrons of the target medium by ionization and/or atomic excitation within an element of path $d x$ is given by the stopping power, $S$, (Tai et al., 1997), i.e.,

$$
S=-\frac{d E}{d x}=\frac{4 \pi Z_{P}^{2} Z_{T} e^{4}}{m v^{2}} N\left\{B_{0}-\frac{C(\beta)}{Z_{T}}+Z_{P} L_{P}(\beta)+Z_{P}^{2} L_{T}(\beta)+\frac{1}{2}\left[G\left(M_{P}, \beta\right)-\delta(\beta)\right]\right\}
$$

where

$$
B_{0}=\ln \left(\frac{2 m c^{2} \beta^{2}}{I\left(1-\beta^{2}\right)}\right)-\beta^{2} .
$$

In the above equations, $Z_{P}$ and $Z_{T}$ are the projectile ion charge and the number of electrons per target atom, respectively, $v$ is the projectile velocity, $c$ is the speed of light, $\beta=v / c, N$ is the density of atoms in the target medium, and $I$ is the mean ionization potential of the target medium. The electron charge and mass are denoted, respectively, by $e$ and $m$.

The various terms in (19) have the following interpretation (Tai et al., 1997; Wilson et al., 1991). The $B_{0}$ term is the high-energy asymptotic limit of the stopping power assuming that the orbital electrons of the target atoms can be treated as essentially free electrons. This requires that the projectile's velocity be much greater than the orbital velocities of the bound atomic electrons, which is an inadequate approximation for inner shell electrons of heavy element target media. The $C(\beta) / Z_{T}$ term provides a correction for inner shell electrons. The $L_{P}(\beta)$ term arises from polarization of the target electrons by the incident ion, and is referred to as 


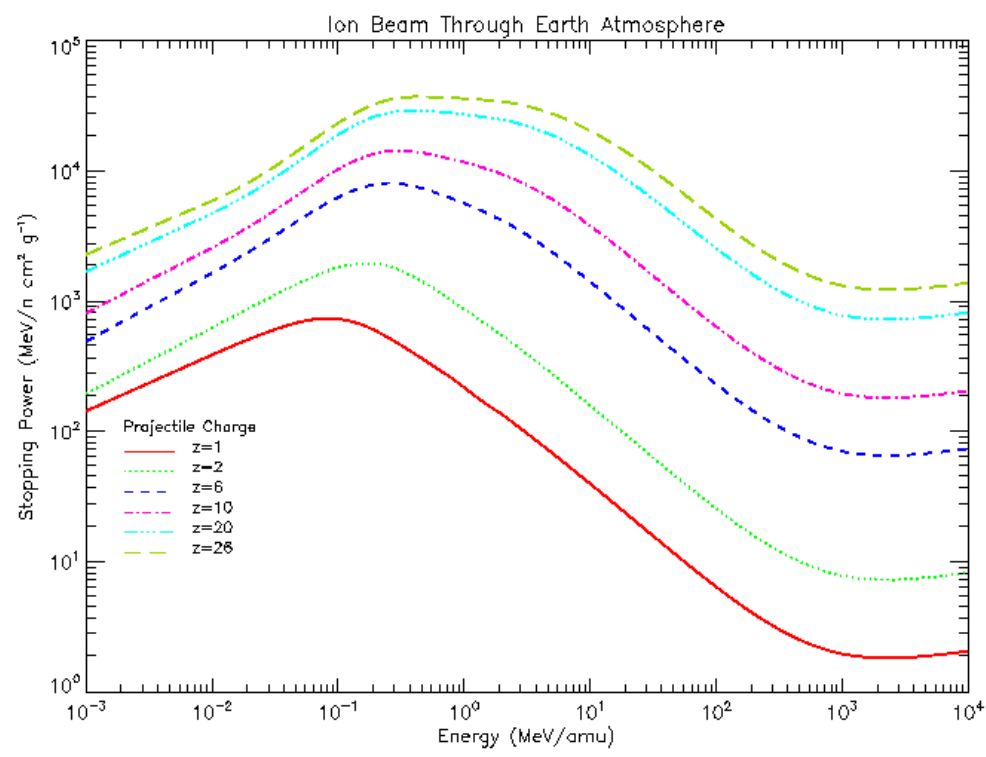

Fig. 17. Stopping power versus kinetic energy of incident ions on Earth's atmosphere. The charge number $(\mathrm{z})$ of the ion is specified in the legend.

the Barkas effect. The $L_{T}(\beta)$ is the Bloch term which provides a correction to the assumption that the ejected orbital electrons in an ionization event can be represented as a plane wave for close collisions with the incident ion. The Mott term is denoted by $G\left(M_{P}, \beta\right)$, where $M_{P}$ is the mass of the projectile ion, which includes a kinetic correction for the recoil of the target nucleus. The $\delta(\beta)$ term is a density correction that originates from the dielectric response of a solid target material to the electric field generated by the projectile ion. Finally, at low energy charge exchange processes begin to dominate, which leads to electron capture by the projectile ion and reduces the atomic excitation and/or ionization energy loss. This effect is included by introducing an effective charge for the projectile ion (Tai et al., 1997).

Figure 17 shows the stopping power for various cosmic ray ions incident on Earth's atmosphere. The stopping power decreases inversely with projectile energy between $\sim 100$ $\mathrm{keV} / \mathrm{amu}$ and $2 \mathrm{GeV} / \mathrm{amu}$. The stopping power begins to increase with increasing projectile energy above $2 \mathrm{GeV} /$ amu due to the relativistic corrections in the $B_{0}$ term in (19). The stopping power decreases for projectile energies less than $\sim 100 \mathrm{keV} / \mathrm{amu}$ due to electron capture by the projectile ion and the other correction terms in (19). The dependence of the stopping power on the projectile kinetic energy plays a major role in determining the spectral shape of the cosmic ray fluence rates in the atmosphere, as indicated by Figures 20 and 21.

The range of an ion is the mean path length traveled in the target medium before coming to rest after losing its initial kinetic energy through ionization and/or atomic excitation energy loss. In the CSDA, the range is defined by

$$
R_{j}(E)=A_{j} \int_{0}^{E} \frac{d E^{\prime}}{S_{j}\left(E^{\prime}\right)}
$$




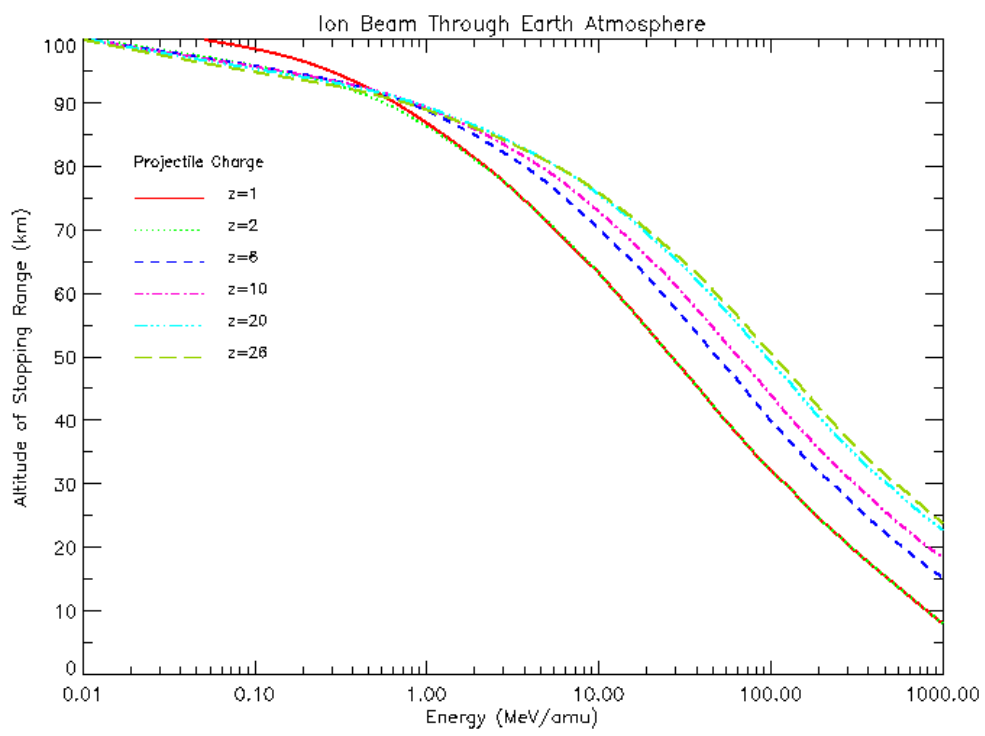

Fig. 18. Average range versus kinetic energy of an ion beam incident at the top of Earth's atmosphere. The charge number $(\mathrm{z})$ of the ion is specified in the legend.

where $A_{j}$ is the atomic mass number of ion particle type $j$. The above equation is referred to as the range-energy relation. Figure 18 shows the range of various cosmic ray ions incident on the neutral atmosphere as a function of ion kinetic energy. At $1 \mathrm{GeV} / \mathrm{amu}$, only protons and alpha particles can reach the typical cruising altitudes of $\sim 10-12 \mathrm{~km}$, or $\sim 200 \mathrm{~g} / \mathrm{cm}^{2}$, for commercial aircraft before coming to rest due to ionization energy loss. Below $1 \mathrm{GeV} / \mathrm{amu}$, all cosmic ray ions loose their kinetic energy before reaching commercial aircraft cruising altitudes. For energies greater than $1 \mathrm{GeV} / \mathrm{amu}$, the cosmic ray particle flux densities decrease with a power-law dependence on energy (see Figure 7). Although secondary charged particles can be produced by nuclear fragmentation reactions, which is represented by the third term in (18), the stopping range in Figure 18 explains why the heavy-ion fluence rates in Figure 20 are significantly less than the proton fluence rate. The same is true for the light-ions in Figure 21.

Two approximations have been made to the total ion-target interaction cross section in (18). First, elastic ion-nucleus scattering has been neglected for cosmic ray transport through the atmosphere. Second, the CSDA has been invoked in the representation of atomic ion-electron energy transfer collisions. As a consequence of these two approximations, the coupled Boltzmann transport equations in (17) can be expressed, alternatively, as

$$
\bar{B}\left[\Phi_{j}(\mathbf{x}, \mathbf{\Omega}, E)\right]=\sum_{k} \iint \sigma_{j k, r}\left(\mathbf{\Omega}, \mathbf{\Omega}^{\prime}, E, E^{\prime}\right) \Phi_{k}\left(\mathbf{x}, \mathbf{\Omega}^{\prime}, E^{\prime}\right) d \mathbf{\Omega}^{\prime} d E^{\prime}
$$

where

$$
\bar{B}\left[\Phi_{j}(\mathbf{x}, \mathbf{\Omega}, E)\right] \equiv\left[\mathbf{\Omega} \bullet \nabla-\frac{1}{A_{j}} \frac{\partial}{\partial E} S_{j}(E)+\sigma_{j, r}(E)\right] \Phi_{j}(\mathbf{x}, \mathbf{\Omega}, E) .
$$

The $\bar{B}\left[\Phi_{j}\right]$ in the above equations denote a differential operator acting on the directional fluence. 
The differential operator in (22) can be inverted using the method of characteristics in order to transform the integro-differential equation into a Volterra-type integral equation (Wilson, 1977). As a result, the integral equation for cosmic ray transport is given by

$$
\begin{aligned}
\Phi_{j}(\mathbf{x}, \mathbf{\Omega}, E)= & \frac{S_{j}\left(E_{\gamma}\right) P_{j}\left(E_{\gamma}\right)}{S_{j}(E) P_{j}(E)} \Phi_{j}\left(\boldsymbol{\Gamma}_{\boldsymbol{\Omega}, \mathbf{x}}, \mathbf{\Omega}, E_{\gamma}\right) \\
+ & \sum_{k} \int_{E}^{E_{\gamma}} \frac{A_{j} P_{j}\left(E^{\prime}\right)}{S_{j}(E) P_{j}(E)} d E^{\prime} \int_{E^{\prime}}^{\infty} d E^{\prime \prime} \int d \mathbf{\Omega}^{\prime} \sigma_{j k, r}\left(\boldsymbol{\Omega}, \mathbf{\Omega}^{\prime}, E^{\prime}, E^{\prime \prime}\right) \\
& \mathbf{x} \Phi_{k}\left[\mathbf{x}+\left(R_{j}(E)-R_{j}\left(E^{\prime}\right)\right) \mathbf{\Omega}, \mathbf{\Omega}^{\prime}, E^{\prime \prime}\right] .
\end{aligned}
$$

In the above equation, $\Gamma_{\Omega, x}$ is a position vector of a point on the boundary surface and $E_{\gamma}$ is given by

$$
E_{\gamma}=R_{j}^{-1}\left[R_{j}+\mathbf{\Omega} \bullet\left(\mathbf{x}-\Gamma_{\Omega, \mathbf{x}}\right)\right]
$$

The $R_{j}^{-1}$ operator in (25) is the inverse operation of obtaining the energy given the range using the range-energy relation in (21). The expression for the integral cosmic ray transport equation in (24) was made compact by introducing the total nuclear survival probability, which is defined by

$$
P_{j}(E) \equiv \exp \left[-A_{j} \int_{0}^{E} \frac{\sigma_{j, r}\left(E^{\prime}\right) d E^{\prime}}{S_{j}\left(E^{\prime}\right)}\right] .
$$

The first term in (24) describes the attenuation of the directional fluence specified at the boundary as a result of transport through the target medium. For atmospheric cosmic ray transport, an isotropic distribution is assumed for the directional fluence and the boundary specification is defined to be the cosmic ray particle fluence rates that have been transported through the heliosphere and magnetosphere and incident at the top of the neutral atmosphere. These incident cosmic ray ions are attenuated by ionization energy loss $(S(E))$ and nuclear absorption $(P(E))$, as indicated by the first term in (24). The second term in (24) describes the generation of type $j$ particles from projectile-target nuclear fragmentation reactions by type $k$ particles. The second term in (24) includes the production of type $j$ particles from type $k$ particles at all intervening positions between the boundary point and the position of observation, accounting for the attenuation by ionization energy loss and nuclear absorption in between the point of production of a type $j$ particle and the observation point.

The representation of the relevant total nuclear absorption cross sections $\left(\sigma_{j, r}\right)$ and nuclear fragmentation production cross sections $\left(\sigma_{j k, r}\right)$ can not be expressed in a simple, compact form such as the stopping power in (19)-(20). Nevertheless, important insight into the influence of nuclear reactions on the atmospheric transport of cosmic rays can be gained by examining the probability of a nuclear reaction as a function of incident ion kinetic energy. The probability of a nuclear reaction is one minus the total nuclear survival probability in (26) (i.e., $1-P_{j}(E)$ ), which is shown in Figure 19. For particles with kinetic energy below $100 \mathrm{MeV} / \mathrm{amu}$, there is a small chance of a nuclear reaction. Recall from Figure 7 that the peak of the incident GCR spectrum is between $\sim 200-500 \mathrm{MeV} / \mathrm{amu}$. At these energies, one out of every two particles will undergo some kind of nuclear reaction. For kinetic energies greater than 1 $\mathrm{GeV} / \mathrm{amu}$, nearly every particle will be subject to some type of nuclear reaction. Combining this discussion with the discussion of Figure 18, the only primary cosmic ray particles that can survive transport through the atmosphere and reach the cruising altitudes of typical commercial aircraft are protons with kinetic energy on the order of $1 \mathrm{GeV}$ or greater. The 


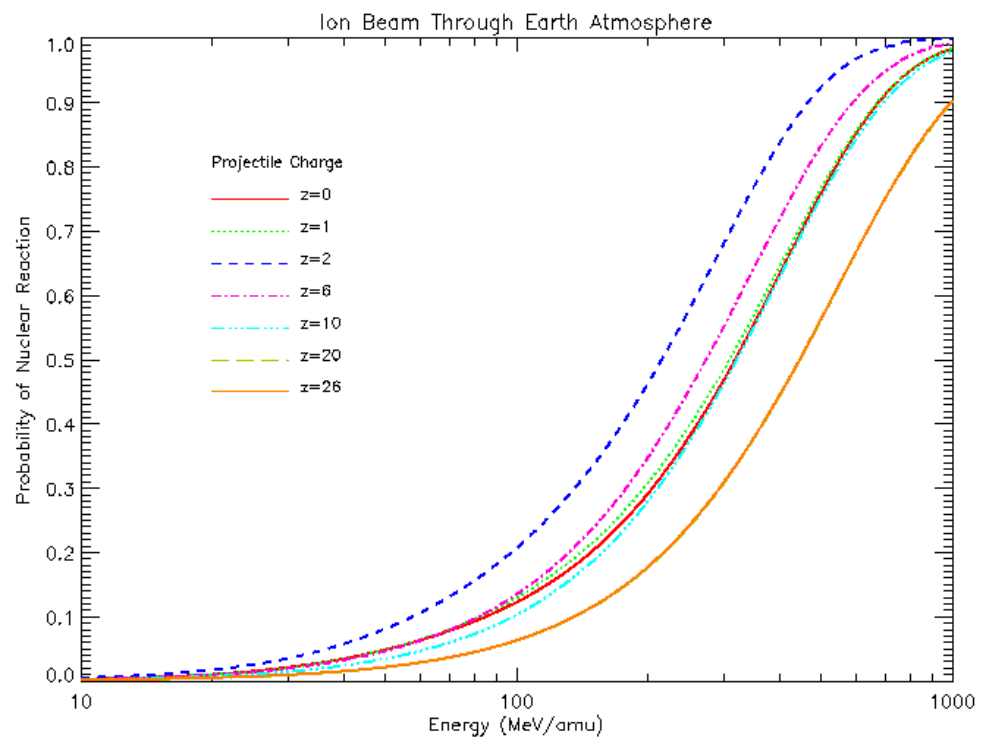

Fig. 19. Nuclear survival probability versus kinetic energy of an ion beam incident at the top of Earth's atmosphere. The charge number $(\mathrm{z})$ of the ion is specified in the legend.

high-LET particles present at 10-12 km with energies less than $1 \mathrm{GeV} / \mathrm{amu}$ are secondary particles created at higher altitudes from nuclear fragmentation reactions, most of which are neutrons.

The coupled cosmic ray integral transport equations in (24) are solved in the NAIRAS model using NASA LaRC's deterministic HZETRN code. Details of the early analytical and computation approaches to solving (24) are given by Wilson et al. (1995a; 1997; 1991; 2005a). The stopping power parameterization used in HZETRN is described by Tai et al. (1997). The nuclear cross sections for neutron and proton interactions are described extensively in Wilson et al. (1989). The model for calculating the heavy-ion nuclear fragmentation cross sections are described by Wilson et al. (1995b). HZETRN is used in a wide variety of radiation transport applications: e.g., the calculation of dosimetric quantities for assessing astronaut risk to space radiations on the International Space Station (ISS) and the Space Transportation System (STS) Shuttle, including realistic spacecraft and human geometry (Badavi et al., 2005; 2007a; Slaba et al., 2009; Wilson \& et al., 2006). Extensive summaries of HZETRN laboratory and space-flight verification and validation are found in recent reports by Badavi et al. (2007a); Nealy et al. (2007); Wilson et al. (2005c;a).

The computation methods employed in HZETRN to solve the coupled cosmic ray integral transport equations in (24) are summarized below. The details are given in the references. The numerical procedures fall into two categories based on the fundamental physics of cosmic ray projectile-target nuclear interactions. The first category is heavy-ion transport. Ion beam experiments have shown that projectile fragments have an energy and direction very near to that of the incident heavy-ion projectile (Wilson, 1977; Wilson et al., 1995a). The observation of forward directed projectile fragments is the bases of the so-called straight-ahead approximation, where the integral over solid angle in (24) is neglected and 

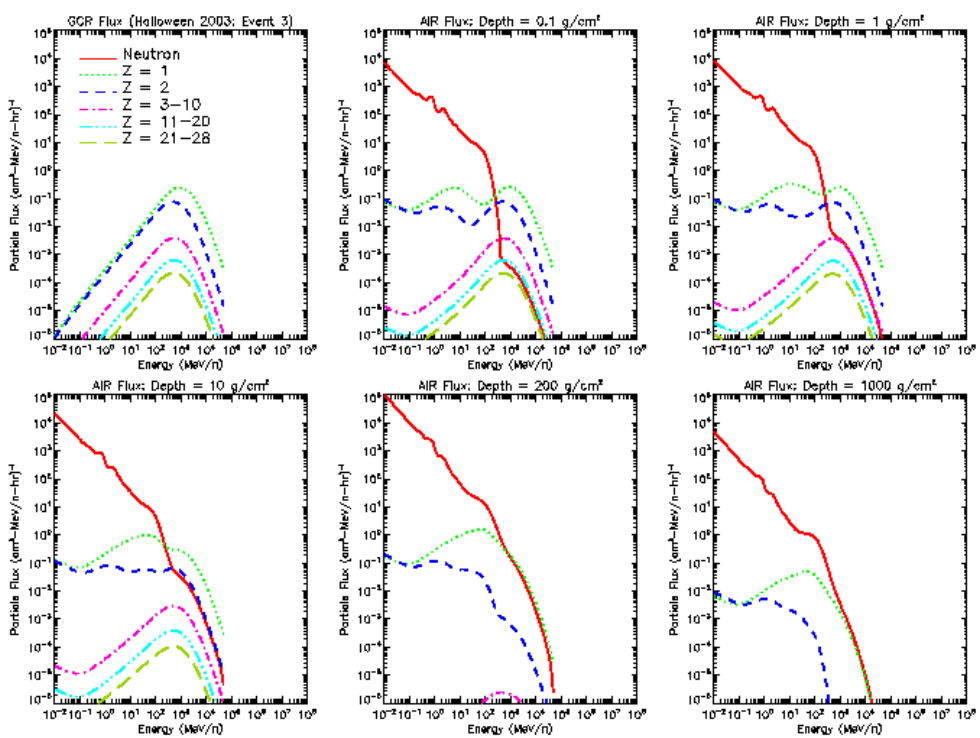

Fig. 20. Event-averaged GCR spectral fluence rates at zero vertical geomagnetic cutoff rigidity during the Halloween 2003 solar-geomagnetic storm [29 October 2003 (2100 UT) to 1 November (0000 UT)]. The panels show the fluence rates at different atmospheric depths. The typical cruising altitudes for commercial aircraft correspond to an atmospheric depth of roughly $200 \mathrm{~g} / \mathrm{cm}^{2}$. The fluence rates from different charge groups have been summed together to reduce the number of lines.

the transport is reduced to one-dimension along the direction of the incident heavy-ion beam. Moreover, the observation of equal velocity between the heavy-ion projectile and the projectile fragment suggests a delta function dependence in the projectile fragment production cross section, which effectively eliminates the integral over $d E^{\prime \prime}$ on the right-hand side of (24).

In addition to the approximations discussed in the previous paragraph, the target fragments are produced at low-energy and distributed nearly isotropically. At low energy, the target fragments do not travel far before coming to rest due to ionization energy loss. These observations justify a decoupling of the target and projectile fragments in the source term on the right-hand side of (24). The advantage of this decoupling is that the target fragments can be neglected in the heavy-ion transport procedure. The absence of the target fragments in the heavy-ion transport solution means that the summation over $k$ type particles in (24) involves only projectiles with masses greater the the mass of the type $j$ particle (i.e., $\sum_{k>j}$ ). These approximations enable a self-consistent solution of projectile fragment heavy-ion transport using backsubstitution and perturbation theory with rapid convergence. The contribution of the target fragments to the dosimetric quantities is included indirectly using the method described in section 3.6. Significant improvements in the accuracy and computational efficiency of HZETRN's heavy-ion numerical transport procedures have recently been made by Slaba et al. (2010c;d).

The numerical solution of the integral transport equation in (24) for light-particle projectiles does not permit the same approximations as the heavy-ion transport solution. Light-particles are defined in HZETRN as those particles with mass number $A \leq 4$ and charge number 

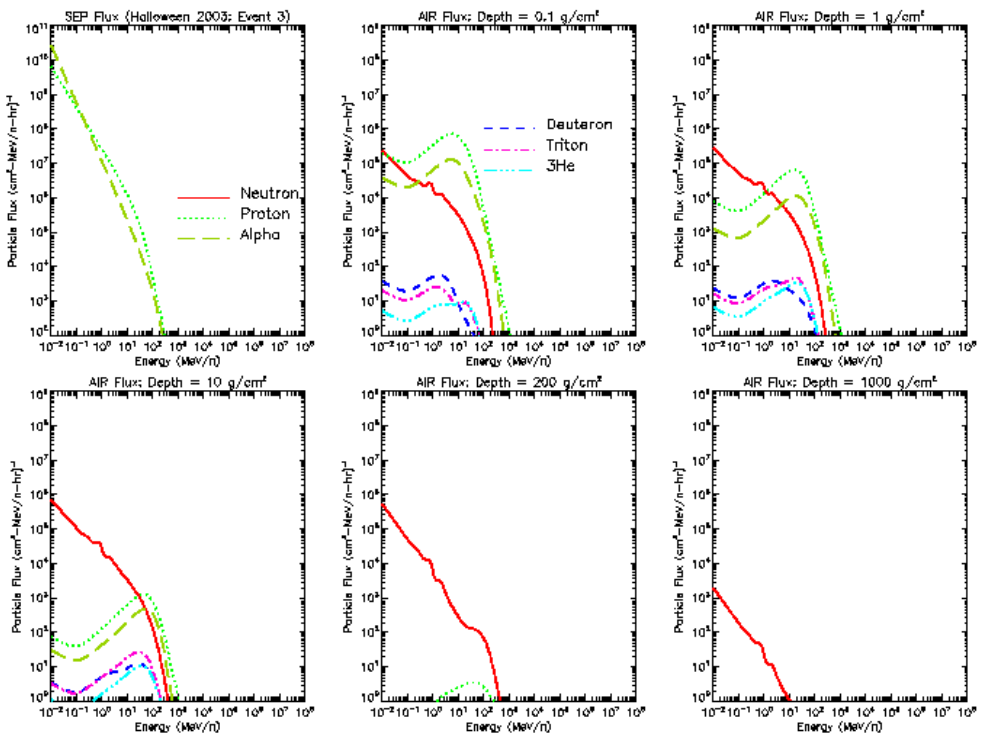

Fig. 21. Event-averaged SEP spectral fluence rates at zero vertical geomagnetic cutoff rigidity during the Halloween 2003 solar-geomagnetic storm [29 October 2003 (2100 UT) to 1

November (0000 UT)]. The panels show the fluence rates at different atmospheric depths. The typical cruising altitudes for commercial aircraft correspond to an atmospheric depth of roughly $200 \mathrm{~g} / \mathrm{cm}^{2}$.

$Z \leq 2$. Thus, there are six light-particles: neutrons and five charged particles (protons, deuterons, tritons, helium-3, and helium-4). The equal velocity relationship between projectile and projectile fragments is no longer valid for light-particle transport. This means that the energy integral over $d E^{\prime \prime}$ on the right-hand side of (24) must be explicitly evaluated, and both the projectile and target fragments are included in the numerical transport procedure. Moreover, the straight-ahead approximation can not be employed in light-particle transport. The integral over solid angle in (24) must be considered, which is especially important for low-energy neutron transport.

Important updates to the numerical solution of light-particle transport in HZETRN have been made recently by Slaba et al. $(2010 \mathrm{a} ; \mathrm{c} ; \mathrm{d} ; \mathrm{b})$. These updates have significantly improved accuracy and computation efficiency. The solution approach is to decompose the light-particle fluence into a straight-ahead component and an isotropic component. The transport solution for the light-particle straight-ahead component is described by Slaba et al. $(2010 \mathrm{c} ; \mathrm{d})$. The neutron fluence is further decomposed into semi-isotropic forward and semi-isotropic backward components. The numerical approach for solving the directionally coupled forward-backward neutron transport scheme for the semi-isotropic component is described by Slaba et al. (2010a;b), which also describes the solution of the charged particle isotropic component. The charged particle isotropic transport is approximated by assuming that the source term originates from nuclear fragmentation reactions between the target medium and the low-energy semi-isotropic neutrons, which turns out to be a good approximation (Slaba et al., 2010c;d). 
In the NAIRAS model, there are 59 coupled transport equations in the HZETRN description of GCR transport through the atmosphere. This set includes transport equations for neutrons and GCR nuclear isotopes from protons through nickel $(Z=28, A=58)$. Figure 20 shows the GCR spectral fluence at various atmospheric depths during the Halloween 2003 storm period. The top left panel shows the spectral fluence at zero vertical cutoff rigidity incident on the neutral atmosphere. The fluences in this panel are the predictions from NAIRAS GCR model (see section 3.1). There is no neutron fluence in this panel since neutrons are secondary particles created by projectile-target nuclear fragmentation reactions, as discussed above. The remaining panels show the GCR fluences (primaries + secondaries) at different depths within the atmosphere.

Since neutrons do not interact with the target medium via the Coulomb force, there is not an ionization threshold, or an atomic excitation threshold, or a nuclear Coulomb potential barrier to overcome. Thus, the neutrons are not brought to rest as the charged particles are. Neutrons continue to cascade down in energy through neutron-nucleus interactions and the low-energy neutron fluence continues to build, as is evident in Figure 20. The low-energy neutron fluence is quite large, even at a small atmospheric depth of $0.1 \mathrm{~g} / \mathrm{cm}^{2}$. The large low-energy neutron fluence at small atmospheric depth is dominated by backscattered neutrons generated at much larger depths, or at much lower altitudes in the atmosphere. At the atmospheric depth of typical cruising altitudes of commercial aircraft, the heavy-ions have largely disappeared, due to a combination of ionization energy loss and nuclear fragmentation reactions into lower energy, lighter particles. Refer to the discussion of Figures 18 and 19.

Solar cosmic rays consist mainly of protons and alpha particles. As a result, only the solution of the six light-particle coupled transport equations defined in HZETRN are required in the description of SEP transport through the atmosphere. Figure 21 shows the light-particle SEP spectral fluences at various atmospheric depths for the same time period during the Halloween 2003 storm as shown in the previous figure. The top left panel shows the spectral fluence at zero vertical cutoff rigidity incident on the neutral atmosphere. These SEP fluence rates were determined using the satellite in-situ ion flux measurements and the spectral fitting algorithm described in section 3.2. The remaining panels show the SEP fluences (primaries + secondaries) at different depths within the atmosphere. Similar to the GCR atmospheric transport properties, the large low-energy neutron fluence at small atmospheric depth for SEP events is due to the large backscattered neutron component, which also originates at larger penetrations depths. Furthermore, only nucleon (protons + neutrons) fluences remain at the typical cruising altitude of commercial aircraft, for the same reasons as previously described.

\subsection{Meteorological data}

This section describes the characterization of the internal properties of the atmosphere that are relevant to cosmic transport. The atmosphere itself provides shielding from incident charged particles. The shielding of the atmosphere at a given altitude depends on the overhead mass. Sub-daily global atmospheric depth is determined from pressure versus geopotential height and pressure versus temperature data derived from the National Center for Environmental Prediction (NCEP) / National Center for Atmospheric Research (NCAR) Reanalysis 1 project (Kalnay \& et al., 1996). The NCAR/NCEP Reanalysis 1 project uses a state-of-the-art analysis/forecast system to perform data assimilation using past data from 1948 to the present. The data products are available $4 x$ daily at $0,6,12$, and 18 UT. The spatial coverage is 17 pressure levels in the vertical from approximately the surface $(1000 \mathrm{hPa})$ to the 


\section{NCAR/NCEP Reanalysis 1: Pressure at $11 \mathrm{~km}$}
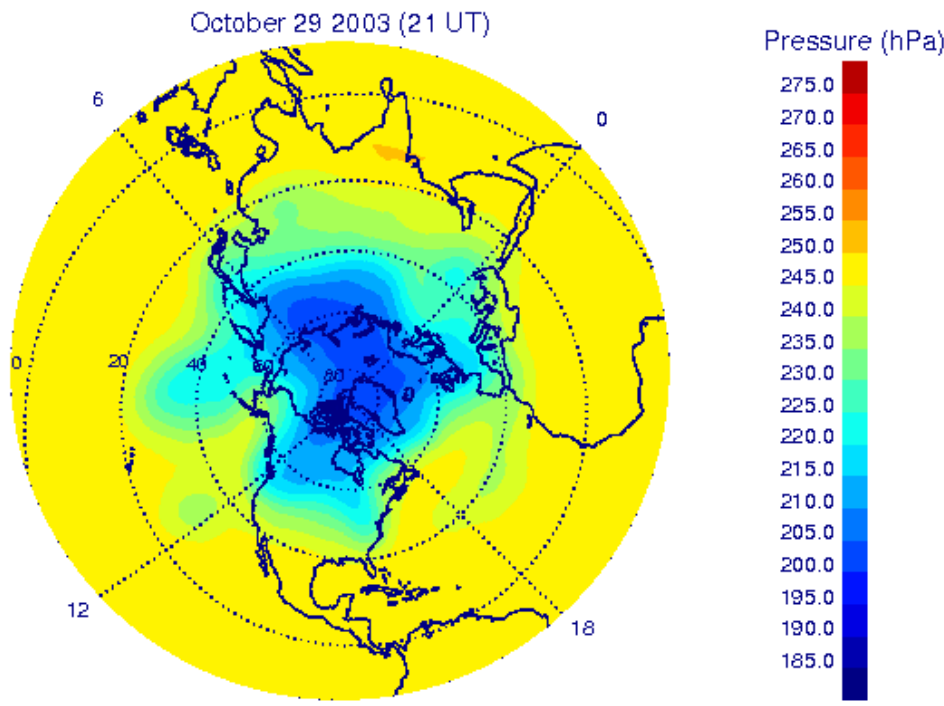

Fig. 22. NCAR/NCEP Reanalysis 1 pressure levels at $11 \mathrm{~km}$ corresponding to the date/time of the largest geomagnetically disturbed period of Halloween 2003 SEP event 3 (10/29/2003, 2100 UT) analyzed in section 4 . Also shown are the magnetic latitude circles and the meridians at $0,6,12$, and 18 magnetic local time.

middle stratosphere $(10 \mathrm{hPa})$, while the horizontal grid is $2.5 \times 2.5$ degrees covering the entire globe.

NCAR/NCEP pressure versus geopotential height data is extended in altitude above $10 \mathrm{hPa}$ using the Naval Research Laboratory Mass Spectrometer and Incoherent Scatter (NRLMSIS) model atmosphere (Picone et al., 2002). NCAR/NCEP and NRLMSIS temperatures are smoothly merged at $10 \mathrm{hPa}$ at each horizontal grid point. NRLMSIS temperatures are produced at $2 \mathrm{~km}$ vertical spacing from the altitude of the NCEP/NCAR $10 \mathrm{hPa}$ pressure surface to approximately $100 \mathrm{~km}$. The pressure at these extended altitudes can be determined from the barometric law using the NRLMSIS temperature profile and the known NCAR/NCEP $10 \mathrm{hPa}$ pressure level, which assumes the atmosphere is in hydrostatic equilibrium and obeys the ideal gas law. Finally, the altitudes and temperatures are linearly interpolated in log pressure to a fixed pressure grid from $1000 \mathrm{hPa}$ to $0.001 \mathrm{hPa}$, with six pressure levels per decade. The result from this step is pressure versus altitude at each horizontal grid point from the surface to approximately $100 \mathrm{~km}$.

Atmospheric depth $\left(\mathrm{g} / \mathrm{cm}^{2}\right)$ at each altitude level and horizontal grid point is computed by vertically integrating the mass density from a given altitude to the top of the atmosphere. The mass density is determined by the ideal gas law using the pressure and temperature at each altitude level. The result from this step produces a 3-D gridded field of atmospheric depth. Atmospheric depth at any specified aircraft altitude is determined by linear interpolation along the vertical grid axis in log atmospheric depth. Figure 22 shows the atmospheric 


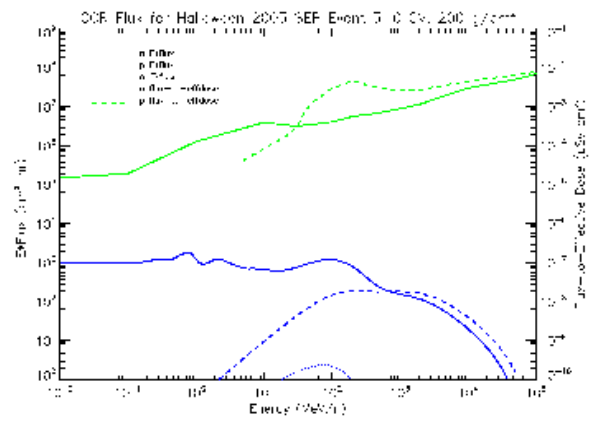

(a) GCR Component

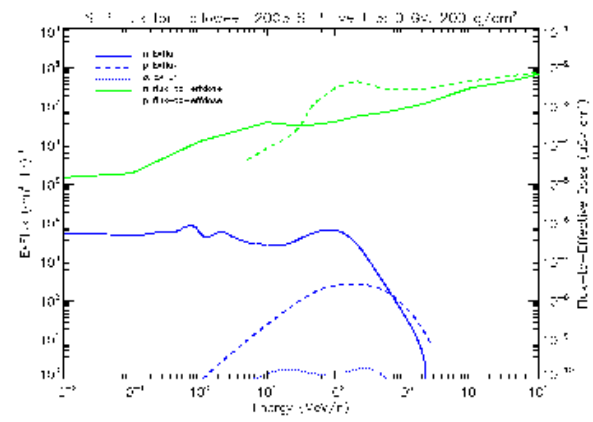

(b) SEP Component

Fig. 23. The green lines show the fluence-to-effective dose conversion coefficients. The blue lines show the product of the kinetic energy (MeV/amu) times the event-averaged spectral fluence rates for the Halloween 2003 storm period [29 October (2100 UT) to 1 November (0000 UT)]. The fluence rates were evaluated at zero vertical geomagnetic cutoff rigidity and at an atmospheric depth of $200 \mathrm{~g} / \mathrm{cm}^{2}$. The lines denote neutron quantities; dashed lines denote proton quantities; dotted lines denote alpha quantities. All quantities are shown for: (a) GCR component and (b) SEP component.

pressure over the northern hemisphere at $11 \mathrm{~km}$ on October 29, 2003 (2100 UT). This is the atmospheric data used in the exposure rate calculations in section 4.3.

\subsection{Radiation dosimetry}

The energy deposited in a target medium by the radiation field of particle $j$ is the dose, which is given by

$$
D_{j}(\mathbf{x})=K \int_{\mathbf{\Omega}} \int_{0}^{\infty} S_{j}(E) \Phi_{j}(\mathbf{x}, \mathbf{\Omega}, E) d \mathbf{\Omega} d E .
$$

In the above equation, $S_{j}(E)$ is the target stopping power for particle $j\left(\mathrm{Mev} / \mathrm{g} / \mathrm{cm}^{2}\right)$ and $K$ is a unit conversion factor $\left(1.602 \times 10^{-10}\right)$ to convert dose to units of Gray $(1 \mathrm{~Gy}=\mathrm{J} / \mathrm{kg})$. The target stopping power is given by (19)-(20), which is shown in Figure 17 for representative cosmic ray ions incident on Earth's atmosphere. Radiation health risk and the probability of biological damage depend not only on the absorbed dose, but also on the particle type and energy of the radiation causing the dose. This is taken into account by weighting the absorbed dose by a factor related to the quality of the radiation. The weighted absorbed dose has been given the name dose equivalent by the ICRP (ICRP, 1991). The unit of dose equivalent is the Sievert (Sv). Dose equivalent in tissue $T$ from particle $j\left(H_{j, T}(\mathbf{x})\right)$ is defined in terms of the tissue LET dependent quality factor $Q$, such that

$$
H_{j, T}(\mathbf{x})=\int_{L} Q(L) D_{j}(\mathbf{x}, L) d L,
$$

where $L$ is LET, which can be approximated by the stopping power in units of $\mathrm{keV} / \mathrm{um}$; $D_{j}(\mathbf{x}, L)$ is the spectral dose distribution from particle $j$ in terms of LET, and $Q(L)$ is the tissue LET-dependent quality factor. 
The relationship between the probability of biological damage and dose equivalent is found to also depend on the organ or tissue irradiated. A further dosimetric quantity, called the effective dose, is defined to include the relative contributions of each organ or tissue to the total biological detriment caused by radiation exposure. The effective dose $(E(\mathbf{x}))$ is the sum of weighted dose equivalents in all the organs and tissues in the human body, such that

$$
E(\mathbf{x})=\sum_{T} \sum_{j} w_{T} H_{j, T}(\mathbf{x}) .
$$

The organ/tissue weighting factors are given in the ICRP 60 report (ICRP, 1991). A computationally efficient approach is to calculate the effective dose rates directly from the particle spectral fluence rates using pre-computed fluence-to-effective dose conversion coefficients. The NAIRAS model uses neutron and proton conversion coefficients tabulated by Ferrari et al. (1997a;b). The effective dose contributions from the other ions are obtained by scaling the proton fluence-to-effective dose conversion coefficients by $Z_{j}^{2} / A_{j}$, according to stopping power dependence on charge and mass in (19)-(20). All recommended ICRP radiation exposure limits are defined in terms of effective dose.

Figure 23 shows the proton and neutron fluence-to-effective dose conversion coefficients and the event-averaged GCR and SEP spectral fluence rates computed during the Halloween 2003 SEP event 3. The fluence rates were computed at zero vertical geomagnetic cutoff rigidity at an atmospheric depth of $200 \mathrm{~g} / \mathrm{cm}^{2}$, which is the depth corresponding to typical cruising altitudes of 10-12 km for commercial aircraft. Above $20 \mathrm{MeV}$, protons make a larger contribution to effective dose per unit fluence compared to neutrons. Below $20 \mathrm{MeV}$, the reverse is true. The fluence rates are shown in Figure 23 as a product of the fluence rates times the energy. This is a convenient representation on a log-log scale since the spectral integration with respect to log-energy, which is performed in order to obtain the effective dose rate, is proportional to energy multiplied by the fluence rate. In this representation, neutrons dominate below about $1 \mathrm{GeV}$. The peak in energy times the proton fluence rate is slightly larger for the SEP component as compared to the GCR component. At $100 \mathrm{MeV}$ and below, the energy times the neutron fluence rate is nearly an order of magnitude greater for the SEP component compared to the GCR component.

The GCR and SEP normalized spectral and accumulated spectral effective dose rates are presented in Figure 24 for the event-averaged Halloween 2003 SEP event 3. Similar to the previous figure, the effective dose rate quantities were computed at zero vertical geomagnetic cutoff rigidity at an atmospheric depth of $200 \mathrm{~g} / \mathrm{cm}^{2}$. The spectral effective dose rates are normalized with respect to the peak in the spectrum. The peak in both the proton and neutron spectral effective dose rates occur between 100-200 MeV, which is true for both GCR and SEP contributions. Neutrons make the largest contributions to effective dose at energies below the peak in the spectrum. Protons make the largest contribution to effective dose at energies above the peak in the spectrum. The relative spectral contribution of protons and neutrons to effective dose holds for both the GCR and SEP components. Half of the total effective dose rate comes from spectral contributions at energies less than $100 \mathrm{MeV}$ for the GCR component, and at energies less than roughly $20 \mathrm{MeV}$ for the SEP component. Half of the neutron effective dose comes from spectral contributions at energies less than about $30 \mathrm{MeV}$ for the GCR component, which is similar for the SEP component. Recall that the incident GCR and SEP proton fluence rates spectra are very different. For example, compare Figures 7 and 9. As a result, half of the proton effective dose rates comes from spectral contributions at energies less than $100 \mathrm{MeV}$ for the SEP component, and at energies less than about $500 \mathrm{MeV}$ for the GCR component. 


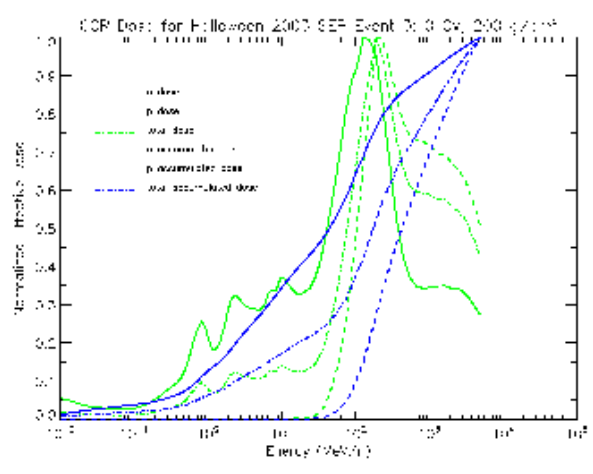

(a) GCR Component

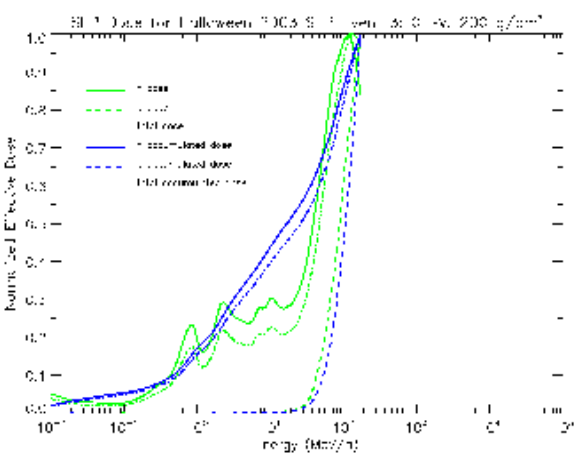

(b) SEP Component

Fig. 24. Normalized spectral effective dose rates evaluated during the Halloween 2003 storm period [29 October (2100 UT) to 1 November (0000 UT)] at zero vertical geomagnetic cutoff rigidity and at an atmospheric depth of $200 \mathrm{~g} / \mathrm{cm}^{2}$. The green lines show the spectral effective dose rate normalized to the peak in the spectrum. The blue lines are normalized to the spectrally integrated effective dose rate, and show the accumulated effective dose rate as the spectral effective dose rate is integrated over energy. The solid lines denote neutron quantities; dashed lines denote proton quantities; dash-dot lines denote total quantities.

\section{Analysis of atmospheric ionizing radiation exposure}

In this section NAIRAS predictions of aircraft radiation exposure are presented and analyzed for three distinct space weather phenomena. The suppression of GCR exposure due to a Forbush decrease is considered briefly in section 4.1. Maximum GCR exposure occurs during solar minimum, as discussed in section 2.1. Results during solar minimum are presented in section 4.2. The Halloween 2003 superstorm is used as a testbed for diagnosing the influence of geomagnetic storm effects on SEP atmospheric radiation exposure. Section 4.3 contains the analysis of the Halloween 2003 SEP exposure and geomagnetic storm effects. When possible, the NAIRAS predictions are compared to other models and/or onboard aircraft radiation measurements.

\subsection{Case Study 1: GCR during forbush decrease}

The Halloween 2003 superstorm was rich in the variety of simultaneous, and often competing, space weather influences (Gopalswamy et al., 2005). Some of the phenomena that occurred during this storm period that exert important influences on atmospheric ionizing radiation exposure are: SEP events, Forbush decreases, geomagnetic storms, cosmic ray anisotropy, and ground level enhancements (GLE). The topic of this section is aircraft radiation exposure during a Forbush decrease that occurred during the Halloween 2003 storm.

A Forbush decrease is a suppression of the GCR exposure due to the interaction of the solar wind with the incident GCR particles. At latitudes with cutoff rigidities greater than 1.0-1.2 GV, the SEP exposure rates are comparable to or less than the GCR exposure rates. Thus, at these latitudes, the total GCR+SEP dose rate can be less than the quiet-time dose rates prior to the SEP event during a Forbush decrease. Getley et al. $(2005 \mathrm{a} ; \mathrm{b})$ observed the apparent influence of a Forbush decrease on dosimetry measurements taken on Qantas Flight 107 from Los Angeles, California to New York, New York on October 29, 2003. These measurements 


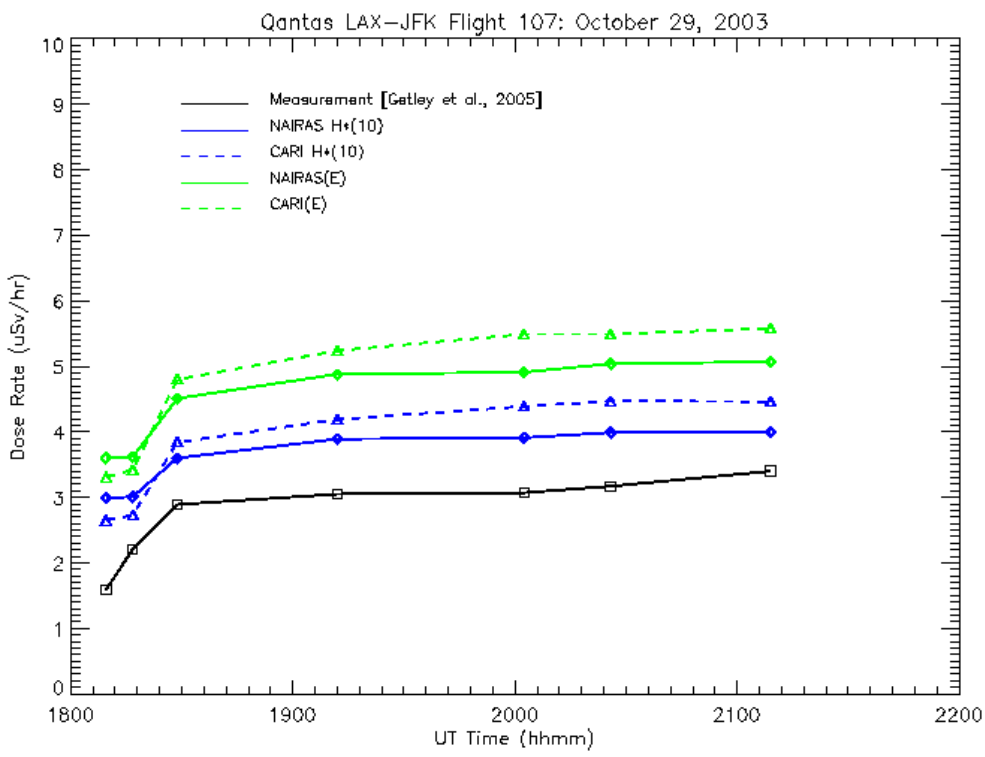

Fig. 25. Comparisons between NAIRAS, FAA/CARI, and TEPC measurements for Qantas Flight 107 from Los Angeles, California to New York, New York on October 29, 2003. The dosimetric quantities compared are effective dose rate (denoted $\mathrm{E}$ ) and ambient dose equivalent rate (denoted $\mathrm{H}^{*}(10)$ ).

are well-suited for testing the ability of NAIRAS to model the reduction in the GCR exposure due to Forbush decreases.

Quantitative comparisons were made between NAIRAS GCR exposure rates and aircraft Tissue Equivalent Proportional Counter (TEPC) measurements taken by Getley et al. (2005a;b). The latitudes of the Qantas flight trajectory were too low to observe significant SEP radiation exposure. However, the GCR exposure was suppressed by a Forbush decrease. Figure 25 shows the comparisons between NAIRAS and the TEPC measurements along the Qantas flight trajectory. This figure shows comparisons of effective dose rate and ambient dose equivalent rate, which is a measurement-based proxy for the effective dose rate. Also shown in the figure are the dosimetric quantities computed from the FAA/CARI-6 model. The NAIRAS calculations are closer to the measurement data than the CARI model. The NAIRAS model can account for Forbush decrease effects by virtue of using real-time neutron monitor count rates in specifying the GCR fluence rates incident at the top of the neutral atmosphere, as discussed in section 3.1.

\subsection{Case Study 2: GCR during solar minimum}

In this section NAIRAS model predictions are compared with TEPC measurements during solar minimum conditions where the GCR exposure reaches its maximum during the solar cycle. TEPC measurements are taken on an equatorial flight and a high-latitude flight. The TEPC data were provided courtesy of Dr. Matthias Meier from The German Aerospace Corporation (DLR) in Cologne, Germany. The equatorial flight was from Dusseldorf, 


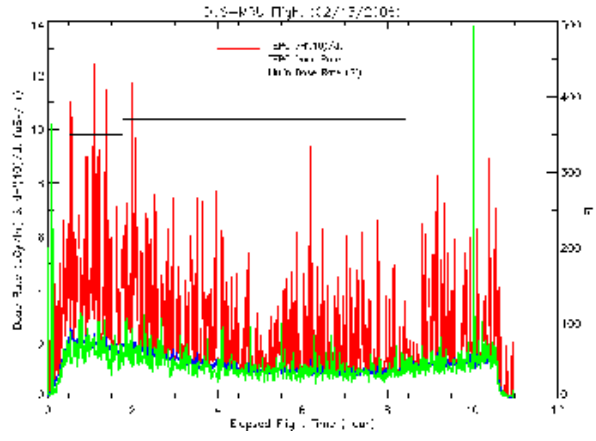

(a) 1-minute TEPC Data

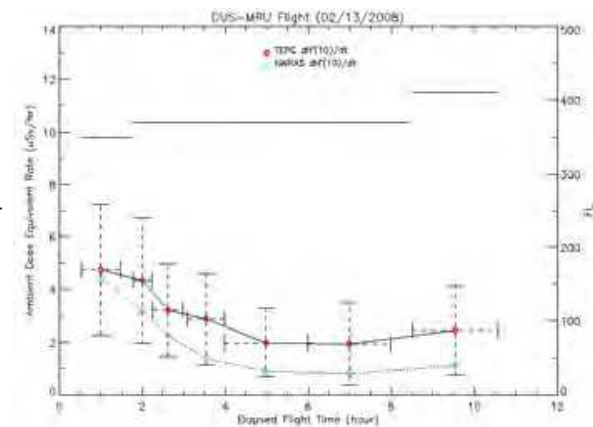

(b) TEPC/NAIRAS Comparison

Fig. 26. (a) TEPC and Liulin dosimetric measurements on a flight from Dusseldorf to Mauritius on February 13, 2008. The left ordinate axis indicate dose rates versus elapsed time of flight (abscissa). The TEPC data shown are: (blue line) absorbed dose rate ( $\mathrm{uGy} / \mathrm{hr}$ ) and (red line) ambient dose equivalent rate (uSv/hr). The Liulin data are (green line) absorbed dose in silicon $(\mathrm{uGy} / \mathrm{hr})$. The right ordinate axis indicates the flight level versus elapsed flight time (abscissa). The flight levels are the horizontal black lines. (b) TEPC/NAIRAS comparisons of ambient dose equivalent rate for the same flight. The left ordinate axis indicates the ambient dose equivalent rate versus elapsed flight time (abscissa). The TEPC measurements are shown as red diamond symbols. The NAIRAS predictions are shown as light blue square symbols. The 1-minute TEPC data shown in the left panel have been averaged over roughly 1-hour periods. The horizontal error bars in the above figure correspond to the averaging interval. The vertical error bars represent one standard deviation in TEPC data.

Germany to the island nation of Mauritius on February 13, 2008, and the TEPC 1-minute data are shown in Figure 26a.

Initial comparison between NAIRAS and TEPC data are focused on the ambient dose equivalent, since this dosimetric quantity is a fairly reasonable proxy for effective dose - the quantity directly related to biological risk. Figure $26 \mathrm{~b}$ shows the NAIRAS/TEPC ambient dose equivalent rate comparisons. The NAIRAS model predictions are within the statistical uncertainty of the TEPC measurements. Nevertheless, NAIRAS results are biased low with respect to the measurements. The largest differences are at the lowest latitudes near the equator. The equatorial low bias in the NAIRAS results may be due to an underprediction of electromagnetic cascade processes initiated by pion production. It is known that the generation and transport of these processes within the HZETRN component of NAIRAS needs improvement, and these processes are likely to be more important at low latitudes compared to higher latitudes. Improvements in HZETRN with modeling the transport and dosimetry of pion initiated electromagnetic cascade effects are currently underway.

The high-latitude dosimetric measurements were taken on a flight from Fairbanks, Alaska to Frankfurt, Germany on May 23, 2008. The 1-minute TEPC data are shown in Figure 27a. NAIRAS/TEPC ambient dose equivalent rate comparisons are shown in Figure 27b. The NAIRAS predictions are at the boundary of one standard deviation in the statistical uncertainty of the TEPC measurements. For the high-latitude comparison, the NAIRAS results are biased high with respect to the TEPC measurements. More comparisons between 


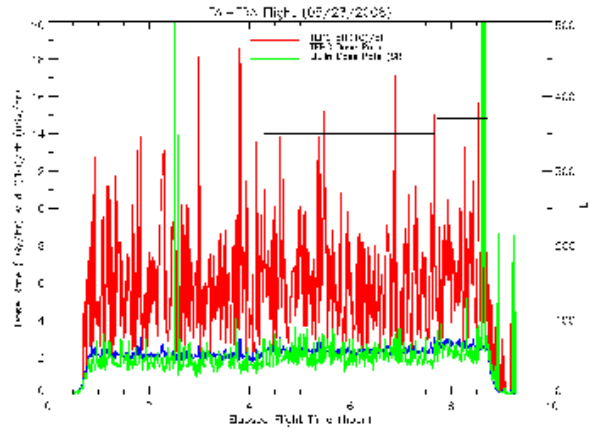

(a) 1-minute TEPC Data

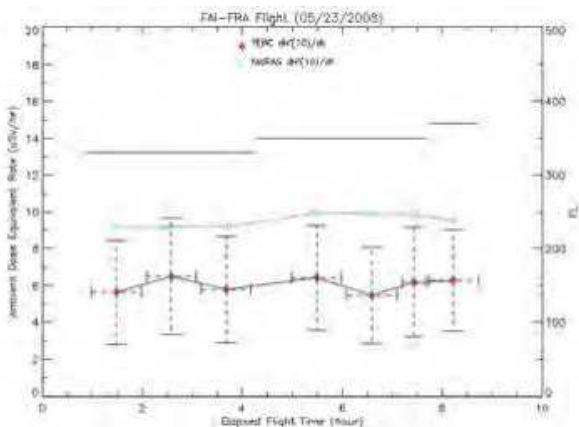

(b) TEPC/NAIRAS Comparisons

Fig. 27. (a) TEPC and Liulin dosimetric measurements on a flight from Fairbanks to Frankfurt on May 23, 2008. The left ordinate axis indicate dose rates versus elapsed time of flight (abscissa). The TEPC data shown are: (blue line) absorbed dose rate (uGy/hr) and (red line) ambient dose equivalent rate (uSv/hr). The Liulin data are (green line) absorbed dose in silicon ( $\mathrm{uGy} / \mathrm{hr})$. The right ordinate axis indicates the flight level versus elapsed flight time (abscissa). The flight levels are the horizontal black lines. (b) NAIRAS/TEPC comparisons of ambient dose equivalent rate for the same flight. The left ordinate axis indicates the ambient dose equivalent rate versus elapsed flight time (abscissa). The TEPC measurements are shown as red diamond symbols. The NAIRAS predictions are shown as light blue square symbols. The 1-minute TEPC data shown in the left panel have been averaged over roughly 1-hour periods. The horizontal error bars in the above figure correspond to the averaging interval. The vertical error bars represent one standard deviation in TEPC data.

NAIRAS predictions and dosimetric measurements over a range of latitudes and solar modulation levels are required to fully characterize the uncertainty in the model predictions. Comprehensive flight campaigns are currently underway for extensive model verification and validation.

\subsection{Case Study 3: A halloween 2003 SEP event}

In this section NAIRAS model predictions are presented for SEP effective dose rates and accumulated effective dose along representative high-latitude commercial routes during the Halloween 2003 SEP event 3 [10/29 (2100 UT) - 10/31 (2400 UT)]. The incident SEP spectral fluence rates and meteorological data are fixed in time in the NAIRAS calculations presented in this section, which are given by the event-averaged spectral fluence rates and atmospheric depth-altitude data shown in Figures 9 and 22, respectively. On the other hand, the geomagnetic cutoff rigidity is allowed to vary in time along the flight trajectories, according to the magnetospheric magnetic field response to the real-time solar wind and IMF conditions (Kress et al., 2010). This enables the geomagnetic influence on SEP radiation exposure to be isolated. The Halloween 2003 superstorm is an ideal event to study geomagnetic effects since this event contained a major magnetic storm which was one of the largest of solar cycle 23. Geomagnetic effects on atmospheric ionizing radiation have not been sufficiently quantified in the past. A unique outcome of this analysis is that it was found that geomagnetic storm effects have a profound effect on SEP atmospheric radiation exposure, especially for flights 


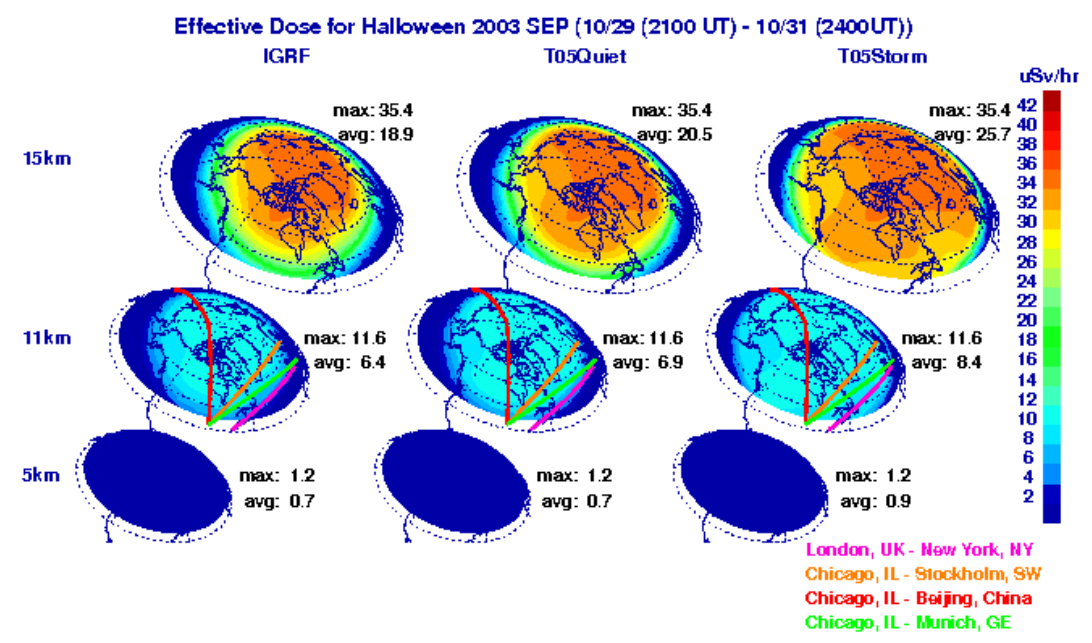

Fig. 28. Effective dose rates computed during Halloween 2003 SEP event 3 . The three columns correspond to exposure rates calculated using the geomagnetic cutoff rigidities and magnetic field models shown in Figure 14. The three rows are exposure rates calculated at different altitudes. In each graph, the hemispheric average effective dose rate (uSv/hr) is indicated by the value next to "avg." The maximum exposure rate is indicated by the value next to "max". See text for definition of "avg" and "max."

along the North Atlantic corridor region connecting international flight from the east coast of the US with Europe. The details of this analysis are given in the section below.

\subsubsection{Global SEP dose distribution}

Global SEP atmospheric ionizing radiation exposure are obtained from a pre-computed database. The effective dose rates are calculated on a fixed 2-D grid in atmospheric depth and cutoff rigidity. The atmospheric depth grid extends from zero to $1300 \mathrm{~g} / \mathrm{cm}^{2}$, and the cutoff rigidity grid extends from zero to $19 \mathrm{GV}$. Both grids have non-uniform spacing with the highest number of grid points weighted toward low cutoff rigidities and tropospheric atmospheric depths. The real-time cutoff rigidities are computed on the same $2.5 \times 2.5$ horizontal grid as the NCEP/NCAR meteorological data. The pre-computed effective dose rates are interpolated to the real-time cutoff rigidity and atmospheric depth specified at each horizontal grid point.

Figure 28 shows global snapshots of atmospheric effective dose rates over the northern hemisphere polar region for the Halloween 2003 SEP event 3. The effective dose rates are shown at three altitudes and for three different magnetic field models used in the cutoff rigidity simulations. The left column shows exposure rates using the IGRF field. The middle column shows exposure rates computed for a geomagnetically quiet time prior to the onset of SEP event 3 using the TS05 field (October 28, 2003, 0002 UT). The right column shows the exposure rates using the TS05 field at the peak of the geomagnetic storm (October 29, 2003, 2100 UT) during SEP event 3. A typical cruising altitude for a commercial high-latitude flight is $11 \mathrm{~km}$. Overlaid on the $11 \mathrm{~km}$ effective dose rate altitude surface are great circle routes for three representative high-latitude commercial flights: London, England (LHR) to New York, 


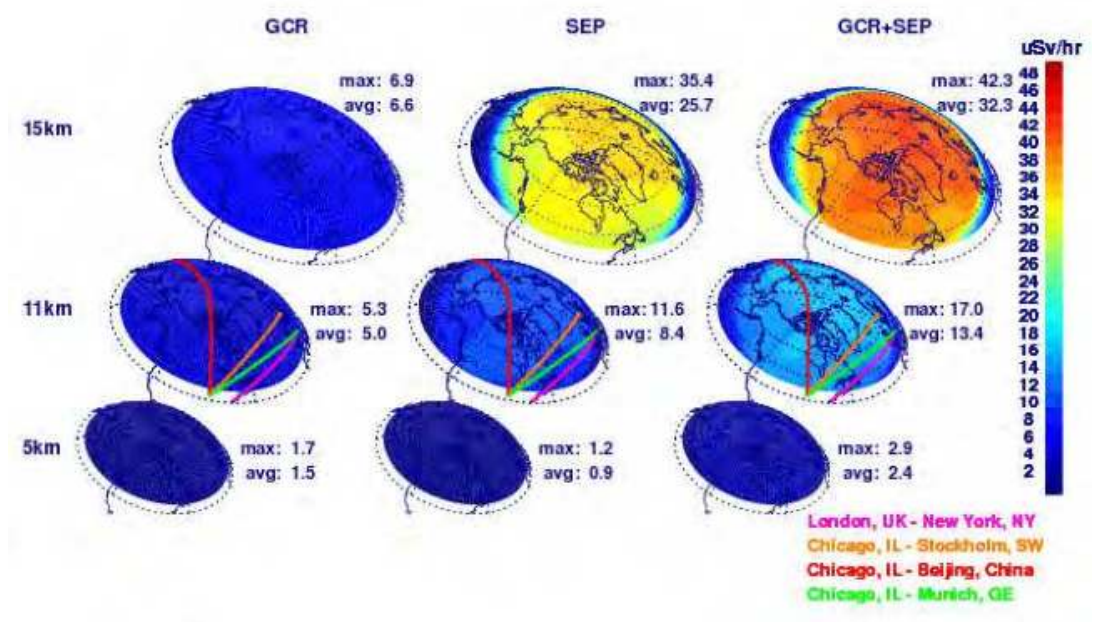

Fig. 29. Effective dose rates computed during Halloween 2003 SEP event 3 . The first, middle, and last columns are the GCR, SEP, and total (GCR+SEP) effective dose rates, respectively. The three rows are exposure rates calculated at different altitudes. In each graph, the hemispheric average effective dose rate (uSv/hr) is indicated by the value next to "avg." The maximum exposure rate is indicated by the value next to "max". See text for definition of "avg" and "max."

New York (JFK) (5.75 hour flight time); Chicago, Illinois (ORD) to Stockholm, Sweden (ARN) (8.42 hour flight time), and a combination of two great circle routes from Chicago, Illinois (ORD) to Beijing, China (PEK) (13.5 hour flight time).

There are a number of striking features to be noted from Figure 28. First, the representation of the geomagnetic field has a significant influence on SEP atmospheric ionizing radiation exposure. Comparing the left and middle columns of Figure 28 shows that even during geomagnetically quiet periods, the magnetospheric magnetic field weakens the overall geomagnetic field with a concomitant increase in radiation levels. This is seen as a broadening of the open-closed magnetospheric boundary in the TS05 quiet field compared to the IGRF field. The cutoffs are zero in the region of open geomagnetic field lines. Thus, effective dose rates based on the IGRF field are underestimated even for magnetically quiet times. During strong geomagnetic storms, as shown in the third column of Figure 28, the area of open field lines are broadened further, bringing large exposure rates to much lower latitudes. Effective dose rates predicted using the IGRF model during a large geomagnetic storm can be significantly underestimated. The expansion of the polar region high exposure rates to lower latitudes, due to geomagnetic effects, is quantified by calculating hemispheric average effective dose rates from $40 \mathrm{~N}$ to the pole. This is denoted by "avg" in Figure 28. At 11 $\mathrm{km}$, there is roughly a $8 \%$ increase in the global-average effective dose rate using TS05 quiet-field compared to IGRF. During the geomagnetic storm, there is a $\sim 30 \%$ increase in the global-average effective dose rate using TS05 storm-field compared to IGRF.

A second important feature to note in Figure 28 is the strong altitude dependence due to atmospheric shielding. The exposure rates are very low at $5 \mathrm{~km}$, independent of geomagnetic field model used. At $15 \mathrm{~km}$, the exposure rates are significantly higher than at $11 \mathrm{~km}$. Figure 28 


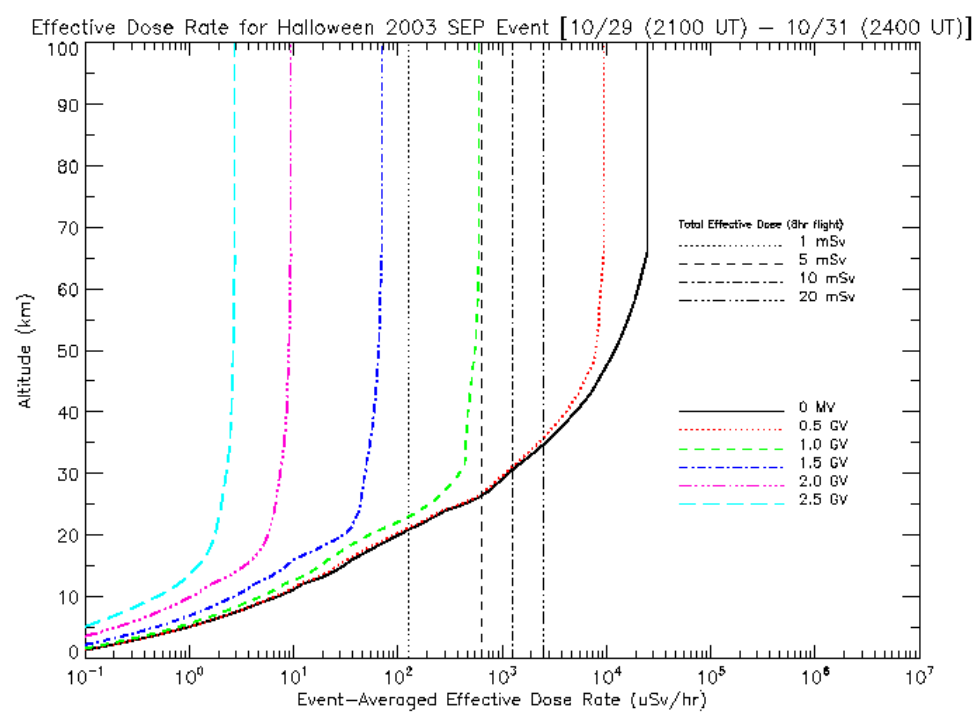

Fig. 30. Event-averaged SEP effective dose rates for Halloween SEP event 3 [(10/29/2003, 2100 UT) - 10/31/2003 (2400 UT)] as a function of altitude for various geomagnetic cutoff rigidities. Different vertical lines indicate constant exposure rates required to reach the corresponding total exposure levels indicated in the legend for a 8-hr flight.

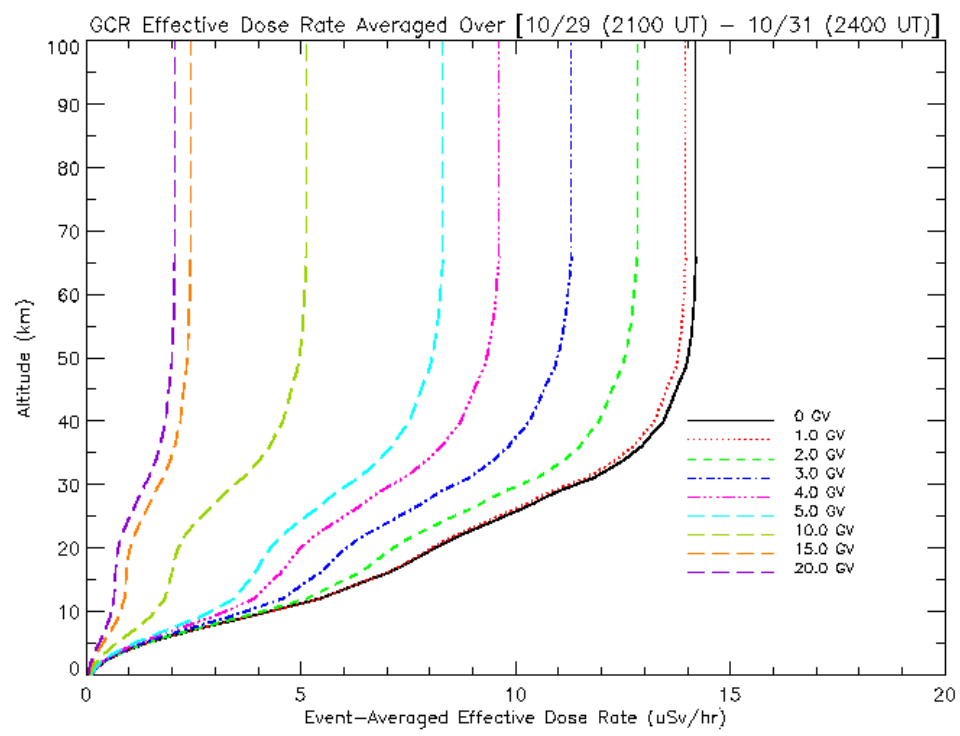

Fig. 31. Event-averaged GCR effective dose rates for Halloween SEP event 3 [(10/29/2003, 2100 UT) $-10 / 31 / 2003$ (2400 UT)] as a function of altitude for various geomagnetic cutoff rigidities. 
shows that the SEP effective dose rates increase (decrease) exponentially with increasing (decreasing) altitude. The SEP exposure rate altitude dependence is a fortunate feature for the aviation community, since radiation exposure can be significantly reduced by descending to lower altitudes. Private business jets will receive more radiation exposure than commercial aircraft if mitigation procedures are not taken, since business jet cruising altitudes are roughly $12-13 \mathrm{~km}$. The altitude dependence of the SEP exposure rates is quantified in Figure 28 by showing the maximum effective dose rate at each altitude, which is the exposure rate at zero cutoff rigidity (i.e., in the polar region of open geomagnetic field lines). The maximum is denoted "max" in Figure 28. The exposure rate increases on average by $160 \%$ per km between $5 \mathrm{~km}$ and $11 \mathrm{~km}$. Between $11 \mathrm{~km}$ and $15 \mathrm{~km}$, the exposure rate increases on average by approximately $75 \%$ per $\mathrm{km}$.

Figure 29 shows the event-averaged GCR and SEP effective dose rates separately for the Halloween 2003 SEP event 3. The total (GCR+SEP) effective dose rates are also shown. The SEP exposure rates were computed with the TS05 field at the peak of the geomagnetic storm (29 October 2003, 2100 UT) during SEP event 3, which are identical to the exposure rates from the third column of Figure 28. At $5 \mathrm{~km}$, the GCR and SEP effective dose rates are comparable. The SEP exposure rates in the polar region at $11 \mathrm{~km}$ are roughly a factor of two greater than the corresponding GCR exposure rates. At $15 \mathrm{~km}$ over the polar region, the SEP exposure rates are greater than the GCR exposure rates by about a factor of five. Due to a substantially larger number of $>100 \mathrm{MeV}$ protons during the January 2005 storm event, the peak polar SEP effective dose rates are significantly greater during the January 2005 event compared to the Halloween 2003 storm period (Copeland et al., 2008).

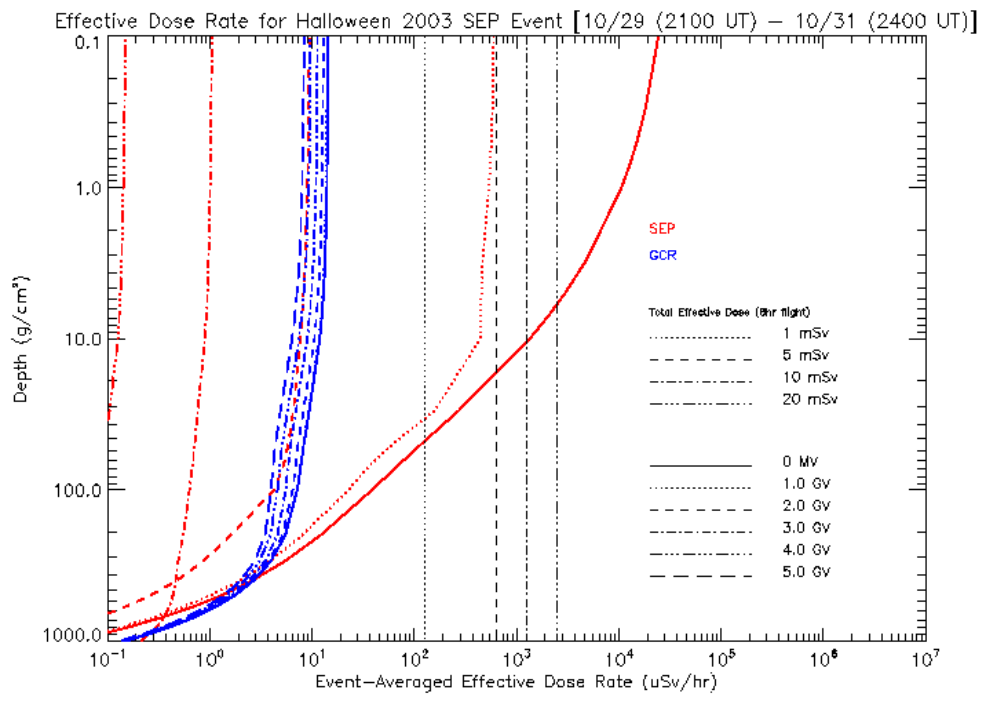

Fig. 32. Event-averaged GCR and SEP effective dose rates for Halloween SEP event 3 [(10/29/2003, 2100 UT) - 10/31/2003 (2400 UT)] as a function of altitude for various geomagnetic cutoff rigidities. 


\subsubsection{Dose on high-latitude flights}

Before calculating radiation exposure along specified flight paths, it's constructive to examine a sample of effective dose rate profiles at different cutoff rigidities from our pre-computed database previously described for SEP event 3. Figure 30 shows SEP effective dose rates as a function of altitude for cutoff rigidities from zero to $2.5 \mathrm{GV}$. This figure clearly shows the exponential dependence of SEP exposure on both cutoff rigidity and altitude. The vertical lines indicate constant exposure rates necessary to receive a total exposure of $1,5,10$, and 20 $\mathrm{mSv}$ on a 8 hour flight. A typical international, high-latitude flight is 8 hours. The rationale for choosing the total exposure identified with the vertical lines is as follows (Wilson et al., 2003): $20 \mathrm{mSv}$ is the ICRP annual occupational radiation worker limit, $10 \mathrm{mSv}$ is the NCRP annual occupational exposure limit, $5 \mathrm{mSv}$ is the NCRP occasional public exposure limit, and $1 \mathrm{mSv}$ is the ICRP annual public and prenatal exposure limit. The cutoff rigidities at high-latitudes are less than $1 \mathrm{GV}$. The typical commercial airline cruising altitudes correspond to an atmospheric depth between $\sim 200-300 \mathrm{~g} / \mathrm{cm}^{2}$. Consequently, one can see from Figure 30 that it's not possible for passengers on high-latitude commercial flights during the Halloween 2003 SEP events to approach or exceed the ICRP public and/or prenatal radiation exposure limit. However, the recommended ICRP annual public and prenatal exposure limits were exceeded during the January 2005 storm event (Copeland et al., 2008).
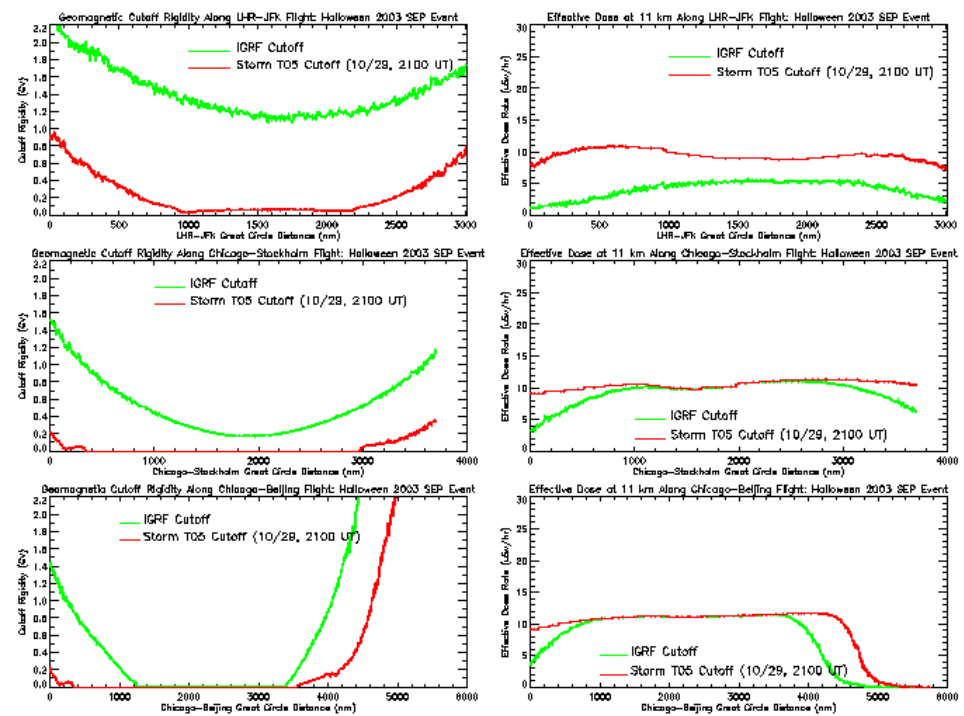

Fig. 33. Geomagnetic cutoff rigidities (left column) and effective dose rates (right column) calculated during Halloween 2003 SEP event 3 along three representative flight paths for a cruising altitude of $11 \mathrm{~km}$. The green line represents cutoff rigidities and exposure rates calculated using the IGRF model. The red lines represent cutoffs and exposure rates computed using the TS05 model during the period of largest geomagnetic activity of event 3 . The total flight times are the following: 5.75 hours from JFK-LHR, 8.42 hours for ORD-ARN, and 13.5 hours for ORD-PEK.

For comparison with the previous plot, Figure 31 shows the GCR effective dose rates as function of altitude for cutoff rigidities from zero to $20 \mathrm{GV}$. In contrast to the SEP exposure 
rates from Figure 30, the GCR exposure rates do not vary exponentially with respect to altitude or cutoff rigidity. The difference between the GCR and SEP exposure rate dependence on cutoff rigidity and altitude is elucidated further by placing both GCR and SEP effective dose rate profiles computed at the same cutoff rigidities on same figure, which is shown in Figure 32. The geomagnetic field is very effective at shielding SEP radiation. For example, at cutoff rigidities greater than $2 \mathrm{GV}$, which for North American corresponds to latitudes south of Canada and the upper regions of the United States, the SEP effective dose rates are already below the GCR exposure rates. The significance of geomagnetic storm effects is that the expansion of the polar cap region, or magnetosphere open-closed boundary, can bring the larger SEP exposure rates, normally confined within the polar cap region, to much lower latitude, even mid-latitudes for a large enough geomagnetic storm.

Figure 33 shows the cutoff rigidities and effective dose rates for the three representative high-latitude flights mentioned in the previous subsection, which were calculated along great circle routes. The left column is the cutoff rigidities along the flight paths and the right column is the corresponding effective dose rates along the flight paths. The cutoff rigidities include both latitude and time-dependent variations along the flight paths. The variations of the exposure rates along the flight paths include latitudinal variations in both atmospheric depth and cutoff rigidity. The temporal variations in cutoff rigidity also map into the variations of the exposure rates along the flight path. The top row shows results for the LHR-JFK flight, while the middle and bottom rows show results for the ORD-ARN and ORD-PEK flights, respectively. Each panel in Figure 33 shows cutoff rigidities and corresponding effective dose rates using the IGRF field (green lines) and the TS05 storm-field (red lines) in the cutoff calculations. The largest differences in flight-path cutoff rigidities between IGRF and TS05 storm-field models are for the LHR-JFK flight. The entire LHR-JFK flight path is near the magnetosphere open-closed boundary and is most sensitive to perturbations in cutoff rigidity due to geomagnetic effects. Consequently, the exposure rates along the LHR-JFK flight are most sensitive to geomagnetic effects. The ORD-PEK polar route is the least sensitive to geomagnetic suppression of the cutoff rigidity, since most of the flight path is across the polar cap region with open geomagnetic field lines. The influence of geomagnetic storm effects on the ORD-ARN flight is intermediate between a typical polar route and a flight along the north Atlantic corridor between the US and Europe.

The effective dose rates for the representative high-latitude flights in Figure 33 are within the range of exposure rates measured during other storm periods for similar flight paths. For example, Clucas et al. (2005) reported peak SEP dose rates in the 3.5-4.0 uSv/hr range for a LHR-JFK flight on July 14, 2000, a SEP event without a concomitant geomagnetic storm. From Figure 33, the peak dose rate for the LHR-JFK flight computed using the IGRF field is $\sim 4 \mathrm{uSv} / \mathrm{hr}$. Clucas et al. also showed peak measured SEP dose rates on the order of 10-12 uSv/hr for a LHR-JFK flight during an April 2001 SEP event, which was accompanied by a geomagnetic storm. The average LHR-JFK effective dose rate in Figure 33 computed using the TS05 storm-field is $9.4 \mathrm{uSv} / \mathrm{hr}$. Dyer et al. (2005) reported measured peak SEP dose rates on the order of $9.5 \mathrm{uSv} / \mathrm{hr}$ for a flight from Prague to New York during the April 2001 SEP event. Thus, our computed effective dose rates are in qualitative agreement with measured dose rates for similar flight paths during other storm periods.

The high sensitivity of SEP atmospheric dose rates to geomagnetic conditions near the open-closed magnetospheric boundary is also responsible for the high sensitivity of SEP dose rates to the exact flight path in the north Atlantic corridor region. For example, Dyer et al. (2007) found that the differences in peak dose rates between great circle and actual flight 
paths for LHR-JFK Concorde flights were a factor of five during the September 1989 SEP event and a factor of 2.5 during the October 1989 event, which was geomagnetically quiet. Furthermore, the difference in peak SEP dose rates between great circle and actual flight paths for the commercial flight from Prague to New York during the April 2001 event was a factor of two.

The actual flight paths for the Chicago to Munich flights reported by Beck et al. (2005) may have been equatorward of a great circle route, which could explain their low dose rates compared to our calculated LHR-JFK great circle route dose rates during the Halloween 2003 superstorm. During the Halloween 2003 SEP event 3, the Chicago-Munich flight measured a mean SEP dose rate of $3.6 \mathrm{uSv} / \mathrm{hr}$ and an accumulated dose of $0.032 \mathrm{mSv}$ for the 8.75 hour flight. These results are comparable to our 5.75 hour JFK-LHR for geomagnetically quiet conditions presented in Figure 33. In other words, the Chicago-Munich flight didn't seem to experience the dose rate enhancement due to the large geomagnetic storm. By comparing the LHR-JFK dose rates in Figure 33 computed using the IGRF field and the TS05 storm-field with the dose rates computed for the ORD-ARN and ORD-PEK flights, it is clear that geomagnetic effects enable flights along the North Atlantic corridor, or near the magnetosphere open-closed boundary, to experience the same dose rates that are confined to the polar region under geomagnetically quiet conditions.

Another possible explanation for the relative difference in the Chicago-Munich measured dose rates reported by Beck et al. (2005) and our computed dose rates for the LHR-JFK flight is our use of the event-averaged incident SEP spectral fluence rate, which we employed for the purpose of isolating geomagnetic effects in our case study. It is clear from the shaded regions in Figure 9 that our event-averaged SEP spectra fluence rate is weighted more toward the peak ion flux measurements observed during SEP event 3 . The measurements in Figure 8 show that the SEP ion flux rapidly decreased in time from the peak values present at the beginning of event 3. Thus, our use of an event-averaged incident SEP spectral fluence rate will tend to overestimate the accumulated effective dose over the flight-paths in Figure 33. However, employing this constraint on the incident SEP spectral fluence rate is necessary to unambiguously isolate the geomagnetic effects. Despite these caveats, the results discussed in this paper are within the current factor of two uncertainty in SEP atmospheric dose rates (Clucas et al., 2005).

\section{Conclusions}

The NAIRAS (Nowcast of Atmospheric Ionizing Radiation for Aviation Safety) prototype operational model is currently streaming live from the project public website (google NAIRAS). NAIRAS predicts biologically hazardous radiation exposure globally from the surface to $100 \mathrm{~km}$ in real-time. The NAIRAS model addresses an important national need with broad societal, public health, and economic benefits. Commercial aircrew are classified by European and international agencies as radiation workers, yet they are the only occupational group exposed to unquantified and undocumented levels of radiation. Furthermore, the current guidelines for maximum public and prenatal exposure can be easily exceeded during a single solar storm event for commercial passengers on intercontinental or polar routes, or by frequent use of these high-latitude routes even during background conditions. The NAIRAS model will provide a new decision support system that currently does not exist, but is essential for providing the commercial aviation industry with data products that will enable airlines to achieve the right balance between minimizing flight cost while at the same time minimizing radiation risk. 
NAIRAS is a physics-based model that maximizes the use of real-time input data. GCR are transported from outside the heliosphere to $1 \mathrm{AU}$ using real-time measurements of ground-based neutron monitor count rates. The SEP particle spectra are determined in-situ using a combination of NOAA/GOES and NASA/ACE ion flux measurements. Both sources of cosmic rays, galactic and solar, are transported through Earth's magnetosphere using a semi-physics-based geomagnetic shielding model. The geomagnetic shielding model utilizes real-time NASA/ACE solar wind and IMF measurements. The cosmic rays are transported from the magnetosphere through the neutral atmosphere using the NASA LaRC's HZETRN deterministic transport code. The real-time, global atmospheric mass density distribution is obtained from the NOAA Global Forecasting System. Global and flight path radiation exposure visualization and decision data products have been developed, which are available at the NAIRAS website.

Future research will focus on new science questions that emerged in the the development NAIRAS prototype operational model (Mertens et al., 2010c). The science questions identified by Mertens et al. (2010c) must be addressed in order to obtain a more reliable and robust operational model of atmospheric radiation exposure. Addressing these science questions require improvements in both space weather modeling and observations.

The Automated Radiation Measurements for Aviation Safety (ARMAS) is a new initiative to address the deficiencies in observations needed to improve the reliability and robustness of operational aircraft radiation exposure assessment. The ultimate goal of the ARMAS initiative is to integrate onboard radiation instruments into a global fleet of aircraft so that the radiation measurements can be downlinked in real-time and assimilated into the NAIRAS predictions of radiation exposure. A subsidiary goal of ARMAS is to provide a testbed to evaluate the accuracy and reliability of new generations of smaller, cheaper hardware technologies in measuring the atmospheric ionizing radiation field. The ARMAS initiative enables the NAIRAS model to adopt the successful meteorological paradigm for reliable and robust weather forecast, which is physics-based models combined with real-time data assimilation of meteorological fields. The NAIRAS/ARMAS approach is a space weather version of terrestrial weather forecasts. These efforts will occupy the NAIRAS team for the next decade or more.

Other research topics unrelated to biological risk from cosmic rays will also be addressed. Effort will also be directed toward predicting the risk that cosmic rays may pose to the operation of microelectronics instrumentation onboard aircraft, and the potential influence cosmic rays may have on the chemistry and climate of planetary atmospheres.

\section{References}

AMS (2007). Integrating space weather observations \& forecasts into aviation operations, Technical report, American Meteorlogical Society Policy Program \& SolarMetrics.

Anderson J. L., Waters, M. A, Hein, M. J., Schubauer-Berigan, M. K. \& Pinkerton, L. E. (2011). Assessment of occupational cosmic radiation exposure of flight attendants using questionnaire data, Aviat Space Environ Med, 82: 1049-54.

Aspholm, R., Lindbohm, M. L., Paakkulainen, H., Taskinen, H., Nurminen, T. \& Tiitinen, A. (1999). Spontaneous abortions among finnish flight attendants, J. Occupational $\mathcal{E}$ Environmental Medicine 41(6): 486-491.

Badavi, F. F., Nealy, J. E., de Angelis, G., Wilson, J. W., Clowdsley, M. S., Luetke, N. J., Cuncinotta, F. A., Weyland, M. D. \& Semones, E. J. (2005). Radiation environment 
and shielding model validation for cev design, Space 2005, number AIAA 2005-6651, Am. Inst. of Aeronaut. and Astronaut., Long Beach, California.

Badavi, F. F., Tramaglina, J. K., Nealy, J. E., \& Wilson, J. W. (2007a). Low earth orbit radiation environments and and shield model validation for iss, Space 2007, number AIAA 2007-6046, Am. Inst. of Aeronaut. and Astronaut., Long Beach, California.

Badhwar, G. D. \& O'Neill, P. M. (1991). An improved model of galactic cosmic radiation for space exploration missions, 22nd International Cosmic Ray Conference, number OG-5.2-13, pp. 643-646.

Badhwar, G. D. \& O'Neill, P. M. (1992). An improved model of galactic cosmic radiation for space exploration missions, Nuclear Tracks Radiat. Meas., 20: 403-410.

Badhwar, G. D. \& O'Neill, P. M. (1993). Time lag of twenty-two year solar modulation, 23nd International Cosmic Ray Conference, Vol. 3, pp. 535-539.

Badhwar, G. D. \& O'Neill, P. M. (1994). Long term modulation of galactic cosmic radiation and its model for space exploration, Adv. Space Res. 14: 749-757.

Badhwar, G. D. \& ONeill, P. M. (1996). Galactic cosmic radiation model and its applications, Adv. Space Res. 17: 7-17.

Baker, D. N., Mason, G. M., Figueroa, O., Colon, G., Watzin, J. G. \& Aleman, R. M. (1993). An overview of the solar, anomalous, and magnetospheric particle explorer (sampex) mission, IEEE Trans. Geosci. Remote Sen. 31(3): 531-541.

Band, P. R., Spinelli, J. J., Ng, V. T. Y., Moody, J. \& Gallagher, R. P. (1990). Mortality and cancer incidence in a cohort of commercial airline pilots, Aviat. Space Environ. Med. 61: 299-302.

Barish, R. J. (1990). Health physics concerns in commercial aviation, Health Phys. 59: 199-204.

Barish, R. J. (2004). In-flight radiation exposure during pregnancy, Obstet. Gynecol. 103: $1326-1330$.

Beck, P., Latocha, M., Rollet, S. \& Stehno, G. (2005). Tepc reference measurements at aircraft altitudes during a solar storm, Adv. Space Res. 36: 1627-1633.

BEIR V (1990). National Researach Council. Health effects of exposure to low levels of ionizing radiation, Washington, DC, National Academy Press.

Bramlitt, E. T. (1985). Commercial aviation crewmember radiation doses, Health Phys. 49: 945-948.

Brandt, S. (1999). Data Analysis, Statistical and Computational Methods for Scientists and Engineers, Springer-Verlag, New York.

Buja, A., Lange, J. H, Perissinotto, E., Rausa, G., Grigoletto, F., Canova, C. \& Mastrangelo, G. (2005) . Cancer incidence among male military and civil pilots and flight attendants: an analysis on published data, Toxicol Ind Health 21: 273-82.

Buja, A., Mastrangelo, G., Perissinotto, E., Grigoletto, F., Frigo, A. C., Rausa, G., Marin, V., Canova. C. \& Dominici, F. (2006). Cancer incidence among female flight attendants: a meta-analysis of the published data, J Womens Health 15: 98-105.

Chen, J., Lewis, B. J., Bennett, L. G. I., Green, A. R. \& Tracy, B. L. (2005). Estimated neutron dose to embryo and foetus during commercial flight, Radiat. Prot. Dosim. 114(4): 475-480.

Clem, J., Clements, D. P., Esposito, J., Evenson, P., Huber, D., LHeureux, J., Meyer, P. \& Constantin, C. (1996). Solar modulation of cosmic electrons, Astrophys. J. 464: 507.

Clucas, S. N., Dyer, C. S. \& Lei, F. (2005). The radiation in the atmosphere during major solar particle events, Adv. Space Res. 36: 1657-1664.

Copeland, K., Sauer, H. H., Duke, F. E. \& Friedberg, W. (2008). Cosmic radiation exposure on aircraft occupants on simulated high-latitude flights during solar proton events from 1 january 1986 through 1 january 2008, Adv. Space Res. 42: 1008-1029. 
DOC (2004). Intense space weather storms october 19 - november 07, 2003, Service assessment, U.S. Department of Commerce, National Oceanic and Atmospheric Administration, National Weather Service, Silver Spring, Maryland.

Dyer, C., Hands, A., Lei, F., Truscott, P., Ryden, K. A., Morris, P., Getley, I., Bennett, L., Bennett, B. \& Lewis, B. (2009). Advances in measuring and modeling the atmospheric radiation environment, IEEE Trans. Nucl. Sci. 56(8).

Dyer, C. \& Lei, F. (2001). Monte carlo calculations of the influence on aircraft radiation environments of structures and solar particle events, IEEE Trans. Nucl. Sci. 48(6): 1987-1995.

Dyer, C., Lei, F., Hands, A., Clucas, S. \& Jones, B. (2005). Measurements of the atmospheric radiation environment from cream and comparisons with models for quiet time and solar particle events, IEEE Trans. Nucl. Sci. 52(6): 2326-2331.

Dyer, C., Lei, F., Hands, A. \& Truscott, P. (2007). Solar particle events in the qinetiq atmospheric radiation model, IEEE Trans. Nucl. Sci. 54(4): 1071-1075.

Ellison, D. C. \& Ramaty, R. (1985). Shock acceleration of electrons and ions in solar flares, Astrophys. J. 298: 400-408.

Engelmann, J. J., Ferrando, P., Soutoul, A., Goret, P., Juliusson, E., Koch-Miramond, L., Lund, N., Masse, P., Peters, B., Petrou, N., \& Rasmussen, I. L. (1990). Charge composition and energy spectra of cosmic-ray nuclei for elements from be to ni. results from heao-3-c2, Astron. Astrophys., 233: 96-111.

European Radiation Dosimetry Group (EURADOS) (1996). Exposure of air crew to cosmic radiation. A report of EURADOS Working Group 11, EURADOS Report 1996.01. In: McAulay, I. R, Bartlett, D. T, Dietze, G. \& et al. editors. European Commission Report Radiation Protection 85. Luxembourg: Office for Official Publications of the European Communities.

Fanton, J. W. \& Golden, J. G. (1991). Radiation-induced endometriosis in Macaca mulatta, Radiation Res, 126: 141-146.

Fedder, J. G. L. J. A. \& Mobarry, C. M. (2004). The lyon-fedder-mobarry (lfm) global mhd magnetospheric simulation code, J. of Atmos. and Solar-Terrestrial Phys. 66(15-16): 1333-1350.

Ferrari, A., Pelliccioni, M. \& Pillon, M. (1997a). Fluence to effective dose conversion coefficients for neutrons up to 10 tev, Radiat. Prot. Dos. 71(3): 165-173.

Ferrari, A., Pelliccioni, M. \& Pillon, M. (1997b). Fluence to effective dose and effective dose equivalent conversion coefficients for protons from $5 \mathrm{mev}$ to $10 \mathrm{tev}$, Radiat. Prot. Dos. 71(2): 85-91.

Foelsche, T. (1961). Radiation exposure in supersonic transports, Technical Report TN D-1383, NASA.

Foelsche, T. \& Graul, E. H. (1962). Radiation exposure in supersonic transports, Atompraxis 8: 365-380.

Foelsche, T., Mendell, R. B., Wilson, J. W. \& Adams, R. R. (1974). Measured and calculated neutron spectra and dose equivalent rates at high altitudes: Relevence to sst operations and space research, Technical Report TN D-7715, NASA.

Friedberg, W., Faulkner, D. N., Snyder, L., Jr., E. B. D. \& OBrien, K. (1989). Galactic cosmic radiation exposure and associated health risk for air carrier crewmembers, Aviat. Space Environ. Med. 60: 1104-1108.

Gaisser, T. (1990). Cosmic Rays and Particle Physics, Cambridge University Press.

Getley, I. L., Duldig, M. L., Smart, D. F. \& Shea, M. A. (2005a). Radiation dose along north america transcontinental flight paths during quiescent and disturbed geomagnetic conditions, Space Weather 3(S01004): doi:10.1029/2004SW000110. 
Getley, I. L., Duldig, M. L., Smart, D. F. \& Shea, M. A. (2005b). The applicability of model based aircraft radiation dose estimates, Adv. Space Res. 36: 1638-1644.

Gold, R. E. \& et al. (1998). Electron, proton, and alpha monitor on the advanced composition and explorer satellite, Space Sci. Rev. 86: 541-562.

Gopalswamy, N., Yashiro, S., Liu, Y., Michalek, G., Vourlidas, A., Kaiser, M. L. \& Howard, R. A. (2005). Coronal mass ejections and other extreme characteristics of the 2003 october november solar eruptions, J. Geophys. Res. 110(A09S15): doi:10.1029/2004JA010958.

Grajewski, B., Waters, M. A., Yong, L. C., Tseng, C.-Y., Zivkovich, Z. \& Cassinelli II, R. T. (2011). Airline pilot cosmic radiation and circadian disruption exposure assessment from logbooks and company records, Ann. Occup. Hyg. 55(5): 465-475.

Haggerty, D. K., E. C. R. G. C. H. \& Gold, R. E. (2006). Quantitative comparison of ace/epam data from different detector heads: Implications for noaa rtse users, Adv. Space Res. 38: 995-1000.

Hammer, G. P, Blettner, M \& Zeeb, H. (2009). Epidemiological studies of cancer in aircrew, Radiat Prot Dosimetry 136: 232-9.

Heinrich, W., Roesler, S. \& Schraube, H. (1999). Physics of cosmic radiation fields, Radiat. Prot. Dosim. 86: 253-258.

ICRP (2008). ICRP Publication 103: 2007 Recommendations of the International Commission on Radiological Protection, ISBN 0-7020-3048-1, Elsevier.

ICRP (1991). ICRP Publication 60: 1990 Recommendations of the International Commission on Radiological Protection, Vol. 21(1-3), Pergamon Press.

ICRU (1986). ICRU Report 40: The quality factor in radiation protection, International Commission on Radiation Units and Measurements.

Jiang, T. N., Lord, B. I. \& Hendry, J. H. (1994). Alpha particles are extremely damaging to developing hemopoiesis compared to gamma radiation, Radiat. Res. 137: 380-384.

Kahler, S. W. (2001). Origin and properties of solar energetic particles in space, in P. Song, H. J. Singer \& G. L. Siscoe (eds), Space Weather, American Geophysical Union, Washington, DC.

Kalnay, E. \& et al. (1996). The ncar/ncep 40-year reanalysis project, Bull. Amer. Meteor. Soc. 77: 437-470.

Kress, B. T., Hudson, M. K., Perry, K. L. \& Slocum, P. L. (2004). Dynamic modeling of geomagnetic cutoff for the 23-24 november 2001 solar energetic particle event, Geophys. Res. Lett. 31(L04808): doi:10.1029/2003GL018599.

Kress, B. T., Mertens, C. J. \& Wiltberger, M. (2010). Solar energetic particle cutoff variations during the 28-31 october 2003 geomagnetic storm, Space Weather 8(S05001): doi:10.1029/2009SW000488.

Lambiotte, J. J., Wilson, J. W. \& Filipas, T. A. (1971). Proper-3c: A nucleon-pion transport code, Technical Report TM X-2158, NASA.

Langlais, B. \& Mandea, M. (2000). An igrf candidate geomagnetic field model for epoch 2000 and a secular variation model for 2000-2005, Earth Planets Space 52: 1137-1148.

Lauria, L., Ballard, T. J., Caldora, M., Mazzanti, C. \& Verdecchia, A. (2006). Reproductive disorders and pregnancy outcomes among female flight attedants, Aviation, Space, and Envirnomental Medicine 77(7): 533-559.

Lewis, B. J., Bennett, G. I., Green, A. R., McCall, M. J., Ellaschuk, B., Butler, A. \& Pierre, M. (2002). Galactic and solar radiation exposure to aircrew during a solar cycle, Radiat. Prot. Dosim. 102(3): 207-227.

Lindborg, L., Bartlett, D. T., Beck, P., McAulay, I. R., Schnuer, K., Schraube, H. \& (Eds.), F. S. (2004). Cosmic radiation exposure of aircraft crew: compilation of measured and calculated data. a report of eurados working group 5, European Radiation 
Dosimetry Group, Lexembourg: Office for the Official Publications of the European Communities, European Communities.

Lopate, C. (2004). Private Communication.

Meier, M., Hubiak, M., Matthiä, D., Wirtz, M. \& Reitz, G. (2009). Dosimetry at aviation altitudes, Radiat. Prot. Dos. 136(4): 251-255.

Menn, W. \& et al. (2000). The absolute flux of protons and helium at the top of the atmosphere using imax, Astrophys. J. 533: 281-297.

Mertens, C. J., Kress, B. T., Wiltberger, M., Blattnig, S. R., Slaba, T. S., Solomon, S. C. \& Engel, M. (2010a). Geomagnetic influence on aircraft radiation exposure during a solar energetic particle event in october 2003, Space Weather 8(S03006): doi:10.1029/2009SW000487.

Mertens, C. J., Moyers, M. F., Walker, S. A. \& Tweed, J. (2010b). Proton lateral broadening distribution comparisons between grntrn, mcnpx, and laboratory beam measurements, Adv. Space Res. 45: 884-891.

Mertens, C. J., Tobiska, W. K., Bouwer, D., Kress, B. T., Solomon, S. C., Kunches, J., Grajewski, B., Gersey, B. \& Atwell, W. (2010c). Nowcast of atmospheric ionizing radiation for aviation safety. Submitted as a white paper to The National Academies Decadal Strategy for Solar and Space Physics (Heliophysics) RFI.

Mertens, C. J., Tobiska, W. K., Bouwer, D., Kress, B. T., Wiltberger, M. J., Solomon, S. C. \& Murray, J. J. (2009). Development of nowcast of atmospheric ionizing radiation for aviation safety (nairas) model, 1st AIAA Atmosheric and Space Environments Conference, number AIAA 2009-3633, Am. Inst. of Aeronaut. and Astronaut., San Antonio, Texas.

Mertens, C. J., Wilson, J. W., Blattnig, S. R., Kress, B. T., Norbury, J. W., Wiltberger, M. J., Solomon, S. C., Tobiska, W. K. \& Murray, J. J. (2008). Influence of space weather on aircraft ionizing radiation exposure, 46th Aerospace Sciences Meeting and Exhibit, number AIAA 2008-0463, Am. Inst. of Aeronaut. and Astronaut., Reno, Nevada.

Mertens, C. J., Wilson, J. W., Blattnig, S. R., Solomon, S. C., Wiltberger, M. J., Kunches, J., Kress, B. T. \& Murray, J. J. (2007a). Space weather nowcasting of atmospheric ionizing radiation for aviation safety, 45th Aerospace Sciences Meeting and Exhibit, number AIAA 2007-1104, Am. Inst. of Aeronaut. and Astronaut., Reno, Nevada.

Mertens, C. J., Wilson, J. W., Walker, S. A. \& Tweed, J. (2007b). Coupling of multiple coulomb scattering with energy loss and straggling in hzetrn, Adv. Space Res. 40: 1357-1367.

Mewaldt, R. A., Cohen, C. M. S., Labrador, A. W., Leske, R. A., Mason, G. M., Desai, M. I., Looper, M. D., Mazur, J. E., Selesnick, R. S., \& Haggerty, D. K. (2005). Proton, helium, and electron spectra during the large solar particle events of october-november 2003, J. Geophys. Res. 110(A09S10): doi:10.1029/2005JA011038.

NAS/NRC (1980). National Academy of Sciences/National Research Council: Health effects of exposures to low levels of ionizing radiation, National Academy Press, Washington, DC. Committee on the Biological Effects of Ionizing Radiation, BEIR V.

NCRP (1993). National Council on Radiation Protection and Measurements: Limitations of exposure to ionizing radiation, Vol. 116, National Council on Radiation Protection and Measurements.

NCRP (2009). National Council on Radiation Protection and Measurements: Ionizing Radiation Exposure of the Population of the United States, NCRP Report No. 160, National Council on Radiation Protection and Measurements.

Nealy, J. E., Cucinotta, F. A., Wilson, J. W., Badavi, F. F., Dachev, T. P., Tomov, B. T., Walker, S. A., Angelis, G. D., Blattnig, S. R. \& Atwell, W. (2007). Pre-engineering spaceflight validation of environmental models and the 2005 hzetrn simulations code, Adv. Space Res. 4: 1593-1610. 
Neher, H. V. (1961). Cosmic-ray knee in 1958, J. Geophys. Res. 66: 4007-4012.

Neher, H. V. (1967). Cosmic-ray particles that changed from 1954 to 1958 to 1965, J. Geophys. Res. 72: 1527-1539.

Neher, H. V. (1971). Cosmic rays at high latitudes and altitudes covering four solar maxima, J. Geophys. Res. 76(7): 1637-1651.

Neher, H. V. \& Anderson, H. R. (1962). Cosmic rays at balloon altitudes and the solar cycle, J. Geophys. Res. 67: 1309-1315.

NOAA (2009). National oceanic and atmospheric administration, space weather prediction center. Available from http://www.swpc.noaa.gov/ftpdir/indices/SPE.txt.

O'Brien, K., Friedberg, W., Smart, D. F. \& Sauer, H. H. (1998). The atmospheric cosmic- and solar energetic particle radiation environment at aircraft altitudes, Adv. Space Res. 21: 1739-1748.

O'Brien, K., Smart, D. F., Shea, M. A., Felsberger, E., Schrewe, U., Friedberg, W. \& Copeland, K. (2003). World-wide radiation doseage calculations for air crew memebers, $A d v$. Space Res. 31(4): 835-840.

Ogilvy-Stuart, A. L. \& Shalet, S. M. (1991). Effect of radiation on the human reproductive system, Environ Health Perspect Suppl, 101(Suppl 2): 109-116.

O'Neill, P. M. (2006). Badhwar-oneill galactic cosmic ray model update based on advanced composition explorer (ace) energy spectra from 1997 to present, Adv. Space Res. 37: 1727-1733.

Onsager, T. G. \& et al. (1996). Operational uses of the goes energetic particle detectors, in goes-8 and beyond, in E. R. Washwell (ed.), SPIE Int. Soc. Opt. Eng., Vol. 2812, pp. 281-290.

Parker, E. N. (1965). The passage of energetic charged particles through interplanetary space, Planet. Space Sci. 13: 9-49.

Picone, J. M., Hedin, A. E., Drob, D. P. \& Aikin, A. C. (2002). Nrlmsis-00 empirical model of the atmosphere: Statistical comparisons and scientific issues, J. Geophys. Res. 107(A12): 1468. doi:10/1029/2002JA009430.

Reitz, G., Schnuer, K. \& Shaw, K. (1993). Editorial - workshop on radiation exposure of civil aircrew, Radiat. Prot. Dosim. 48: 3.

Schraube, H., Mares, V., Roesler, S. \& Heinrich, W. (1999). Experimental verification and calculation of aviation route doses, Radiat. Prot. Dosim. 86(4): 309-315.

Slaba, T. C., Blattnig, S. R. \& Aghara, S. K. (2010a). Coupled neutron transport for hzetrn, Radiat. Meas. 45: 173-182.

Slaba, T. C., Blattnig, S. R. \& Badavi, F. F. (2010c). Faster and more accurate transport procedures for hzetrn, J. Comput. Phys. 229: 9397-9417.

Slaba, T. C., Blattnig, S. R. \& Badavi, F. F. (2010d). Faster and more accurate transport procedures for hzetrn, Technical Report TP-2010-216213, NASA.

Slaba, T. C., Blattnig, S. R., Clowdsley, M. S., Walker, S. A. \& Badavi, F. F. (2010b). An improved neutron transport algorithm for hzetrn, Adv. Space Res. 46: 800-810.

Slaba, T. C., Qualls, G. D., Clowdsley, M. S., Blattnig, S. R., Simonsen, L. C., Walker, S. W., \& Singleterry, R. C. (2009). Analysis of mass averaged tissue doses in max, fax, and cam, and caf, Technical Report TP-2009-215562, NASA.

Smart, D. F. \& Shea, M. A. (1994). Geomagnetic cutoffs: A review for space dosimetry calculations, Adv. Space Res. 14(10): 10,787-10,796.

Smart, D. F. \& Shea, M. A. (2005). A review of geomagnetic cutoff rigidities for earth-orbiting spacecraft, Adv. Space Res. 36: 2012-2020.

Störmer, C. (1965). The Polar Aurora, Oxford at the Clarendon Press. 
Tai, H., Bichsel, H., Wilson, J. W., Shinn, J. L., Cucinotta, F. A. \& Badavi, F. F. (1997). Comparison of stopping power and range databases for radiation transport study, Technical Report TP-3644, NASA.

Toffoletto, F. R., Sazykin, S., Spiro, R. W., Wolf, R. A. \& Lyon, J. G. (2004). Rcm meets lfm: initial results of one-way coupling, J. of Atmos. and Solar-Terrestrial Phys. 66(15-16): 1361-1370.

Townsend, L. W., Stephens, D. L., Hoff, J. L., Zapp, E. N., Moussa, H. M., Miller, T. M., Campbell, C. E. \& Nichols, T. F. (2006). The carrington event: Possible doses to crews in space from a comparable event, Adv. Space Res. 38: 226-231.

Townsend, L. W., Zapp, E. N., Jr., D. L. S. \& Hoff, J. L. (2003). Carrington flare of 1859 as a prototypical worst-case solar energetic particle event, IEEE Trans. Nucl. Sci. 50(6): 2307-2309.

Tsyganenko, N. A. (1989). Determination of magnetic current system parameters and development of experimental geomagneitc field models based on data from imp and heos satellite, Planet Space Sci. 37: 5-20.

Tsyganenko, N. A. (2002). A model of the near magnetosphere with dawn-dusk asymmetry: 1. mathematical structure, J. Geophys. Res. 107(A8): 1179. doi:10.1029/2001JA000219.

Tsyganenko, N. A. \& Sitnov, N. I. (2005). Modeling the dynamics of the inner magnetosphere during strong geomagnetic storms, J. Geophys. Res. 110: A03208. doi:10.1029/2004JA010798.

Tylka, A. J., Cohen, C. M. S., Dietrich, W. F., Lee, M. A., Maclennan, C. G., Mewaldt, R. A., Ng, C. K. \& Reames, D. V. (2005). Shock geometry, seed populations, and the origin of variable elemental composition at high energies in large gradual solar particle events, ApJ. 625: 474-495.

Tylka, A. J. \& Lee, M. A. (2006). Spectral and compositional characteristics of gradual and impuslive solar energetic particle events, in solar eruptions and energetic particles, in N. Gopalswamy, R. Mewaldt \& J. Torsi (eds), Solar Eruptions and Energetic Particles, Vol. Geophysical Monograph 165, American Geophysical Union, Washington, DC.

UNSCEAR (1988). UNSCEAR 1988 Report to the General Assembly: Sources, effects, and risks of ionizing radiation, number E.88.IX.7, United Nations Scientific Committee on the Effects of Atomic Radiation, United Nations, New York.

Upton, A. C., Chase, H. B., Hekhuis, G. L., Mole, R. H., Newcombe, H. B., Robertson, J. S., Schaefer, H. J., Synder, W. S., Sondhaus, C. \& Wallace, R. (1966). Radiobiological aspects of the supersonic transport, Health Phys. 12: 209-226.

VanAllen, J. A. (1968). Physics of the Magnetosphere, Vol. 10, Springer-Verlag, New York, New York, chapter Particle Description of the Magnetosphere.

Wallance, R. G. \& Sondhaus, C. A. (1978). Cosmic ray exposure in subsonic air transport, Aviation Space, and Environ. Med. 74: 6494-6496.

Wang, W., Wiltberger, M., Burns, A. G., Solomon, S. C., Killeen, T. L., Maruyama, N. \& Lyon, J. G. (2004). Initial results from the coupled magnetosphereionosphere-thermosphere model: thermosphere-ionosphere responses, J. of Atmos. and Solar-Terrestrial Phys. 66: 1425-1441.

Waters, M., Grajewski, B., Pinkerton, L. E., Hein, M. J. \& Zivkovich, Z. (2009). Development of historical exposure estimates of cosmic radiation and circadian rhythm disruption for cohort studies of Pan- Am flight attendants, Am J Ind Med 52: 751-61.

Waters, M., Bloom, T. F. \& Grajewski, B. (2000). The NIOSH/FAA working womens health study: Evaluation of the cosmic-radiation exposures of flight attendants, Health Phys. 79(5): 553-559. 
Wilson, J. W. (1977). Analysis of the theory of high-energy ion transport, Technical Report TN D-8381, NASA.

Wilson, J. W. (2000). Overview of radiation environments and human exposures, Heath Phys. 79(5): 470-494.

Wilson, J. W., Badavi, F. F., Cucinotta, F. A., Shinn, J. L., Badhwar, G. D., Silberberg, R., Tsao, C. H., Townsend, L. W. \& Tripathi, R. K. (1995a). Hzetrn: Description of a free-space ion and nucleon transport and shielding computer program, Technical Report TP 3495, NASA.

Wilson, J. W., Joes, I. W., Maiden, D. L. \& Goldhagan, P. (eds) (2003). Analysis, results, and lessons learned from the June 1997 ER-2 campaign, NASA CP-2003-212155, NASA Langley Research Center.

Wilson, J. W., Lambiotte, J. J., Foelsche, T. \& Filippas, T. A. (1970). Dose response functions in the atmosphere due to incident high-energy protons with applications to solar proton events, Technical Report TN D-6010, NASA.

Wilson, J. W., Mertens, C. J., Goldhagan, P., Friedberg, W., Angelis, G. D., Clem, J. M., Copeland, K. \& Bidasaria, H. B. (2005b). Atmospheric ionizing radiation and human exposure, Technical Report TP-2005-213935, NASA.

Wilson, J. W., Miller, J. \& Cucinotta, F. A. (eds) (1997). Shielding strategies for human space exploration, NASA Conference Publication 3360, NASA Johson Space Center.

Wilson, J. W. \& Townsend, L. W. (1988). Radiation safety in commercial air traffic: A need for further study, Health Phys. 55: 1001-1003.

Wilson, J. W., Townsend, L. W., Nealy, J. E., Chung, S. Y., Hong, B. S., Buck, W. W., Lamkin, S. L., Ganapol, B. D., Khan, F. \& Cucinotta, F. A. (1989). Bryntrn: A baryon ttansport model, Technical report, NASA.

Wilson, J. W., Townsend, L. W., Schimmerling, W., Khandelwal, G. S., Khan, F., Nealy, J. E., Cucinotta, F. A., Simonsen, L. C., Shinn, J. L., \& Norbury, J. W. (1991). Transport methods and interactions for space radiation, Technical Report RP-1257, NASA.

Wilson, J. W., Tripathi, R. K., Cucinotta, F. A., Shinn, J. L., Badavi, F. F., Chun, S. Y., Norbury, J. W., Zeitlin, C. J., Heilbronn, L. \& Miller, J. (1995b). Nucfrg2: An evalution of the semiempirical nuclear fragmentation database, Technical Report TP-3533, NASA.

Wilson, J. W., Tripathi, R. K., Mertens, C. J., Blattnig, S. R., Clowdsley, M. S., Cucinotta, F. A., Tweed, J., Heinbockel, J. H., Walker, S. A. \& Nealy, J. E. (2005c). Verification and validation of high charge and energy (hze) transport codes and future development, Technical Report TP-2005-213784, NASA.

Wilson, J. W., Tweed, J., Walker, S. A., Cucinotta, F. A., Tripathi, R. K., Blattnig, S. \& Mertens, C. J. (2005a). A benchmark for laboratory exposures with $1 \mathrm{a}$ gev iron ions, Adv. Space Res. 35: 185-193.

Wilson, J. W. \& et al. (2006). International space station: A testbed for experimental and computational dosimetry, Adv. Space Res. 37: 1656-1663.

Xapsos, M. A., Barth, J. L., Stassionopoulos, E. G., Messenger, S. R., Walters, R. J., Summers, G. P. \& Burke, E. A. (2000). Chracterizing solar proton energy spectra for radiation effects applications, IEEE Trans. Nucl. Sci. 47(6): 2218-2223. 


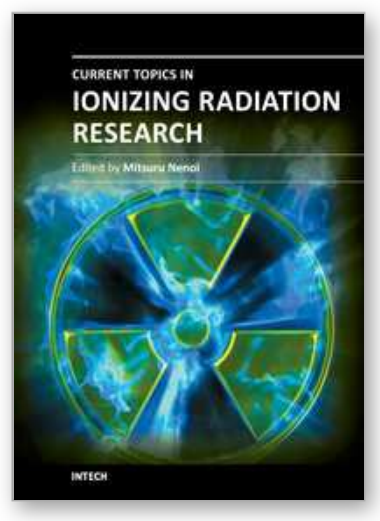

\author{
Current Topics in lonizing Radiation Research \\ Edited by Dr. Mitsuru Nenoi
}

ISBN 978-953-51-0196-3

Hard cover, 840 pages

Publisher InTech

Published online 12, February, 2012

Published in print edition February, 2012

Since the discovery of $X$ rays by Roentgen in 1895 , the ionizing radiation has been extensively utilized in a variety of medical and industrial applications. However people have shortly recognized its harmful aspects through inadvertent uses. Subsequently people experienced nuclear power plant accidents in Chernobyl and Fukushima, which taught us that the risk of ionizing radiation is closely and seriously involved in the modern society. In this circumstance, it becomes increasingly important that more scientists, engineers and students get familiar with ionizing radiation research regardless of the research field they are working. Based on this idea, the book "Current Topics in lonizing Radiation Research" was designed to overview the recent achievements in ionizing radiation research including biological effects, medical uses and principles of radiation measurement.

\title{
How to reference
}

In order to correctly reference this scholarly work, feel free to copy and paste the following:

Christopher J. Mertens, Brian T. Kress, Michael Wiltberger, W. Kent Tobiska, Barbara Grajewski and Xiaojing $\mathrm{Xu}$ (2012). Atmospheric lonizing Radiation from Galactic and Solar Cosmic Rays, Current Topics in lonizing Radiation Research, Dr. Mitsuru Nenoi (Ed.), ISBN: 978-953-51-0196-3, InTech, Available from: http://www.intechopen.com/books/current-topics-in-ionizing-radiation-research/atmospheric-ionizing-radiationfrom-galactic-and-solar-cosmic-rays

\section{INTECH}

open science | open minds

\author{
InTech Europe \\ University Campus STeP Ri \\ Slavka Krautzeka 83/A \\ 51000 Rijeka, Croatia \\ Phone: +385 (51) 770447 \\ Fax: +385 (51) 686166 \\ www.intechopen.com
}

\author{
InTech China \\ Unit 405, Office Block, Hotel Equatorial Shanghai \\ No.65, Yan An Road (West), Shanghai, 200040, China \\ 中国上海市延安西路65号上海国际贵都大饭店办公楼 405 单元 \\ Phone: +86-21-62489820 \\ Fax: $+86-21-62489821$
}


(C) 2012 The Author(s). Licensee IntechOpen. This is an open access article distributed under the terms of the Creative Commons Attribution 3.0 License, which permits unrestricted use, distribution, and reproduction in any medium, provided the original work is properly cited. 